\title{
The Gould's Belt Distances Survey (GOBELINS). IV. Distance, Depth, and Kinematics of the Taurus Star-forming Region
}

\author{
Phillip A. B. Galli ${ }^{1}$ (1), Laurent Loinard ${ }^{2,3}$ (1) , Gisela N. Ortiz-Léon ${ }^{4}$ (D), Marina Kounkel ${ }^{5}$ (D), Sergio A. Dzib ${ }^{4}$ (1), \\ Amy J. Mioduszewski ${ }^{6}$, Luis F. Rodríguez ${ }^{2}$, Lee Hartmann ${ }^{7}$ (i), Ramachrisna Teixeira ${ }^{1}$, Rosa M. Torres ${ }^{8}$, Juana L. Rivera ${ }^{2}$ (i), \\ Andrew F. Boden ${ }^{9}$, Neal J. Evans II $^{10,11,12}{ }^{\text {, Cesar Briceño }}{ }^{13}$, John J. Tobin ${ }^{14}{ }^{(1)}$, and Mark Heyer ${ }^{15}$ \\ ${ }^{1}$ Instituto de Astronomia, Geofísica e Ciências Atmosféricas, Universidade de São Paulo, Rua do Matão, 1226, Cidade Universitária, \\ 05508-900, São Paulo-SP, Brazil; phillip.galli@iag.usp.br \\ ${ }^{2}$ Instituto de Radioastronomía y Astrofísica, Universidad Nacional Autónoma de México, Apartado Postal 3-72, Morelia 58089, México \\ ${ }^{3}$ Instituto de Astronomía, Universidad Nacional Autónoma de México, Apartado Postal 70-264, 04510 Ciudad de México, México \\ ${ }^{4}$ Max Planck Institut für Radioastronomie, Auf dem Hügel 69, D-53121, Bonn, Germany \\ ${ }^{5}$ Department of Physics and Astronomy, Western Washington University, 516 High St., Bellingham, WA 98225, USA \\ ${ }^{6}$ National Radio Astronomy Observatory, Domenici Science Operations Center, 1003 Lopezville Road, Socorro, NM 87801, USA \\ ${ }^{7}$ Department of Astronomy, University of Michigan, 1085 S. University St., Ann Arbor, MI 48109, USA

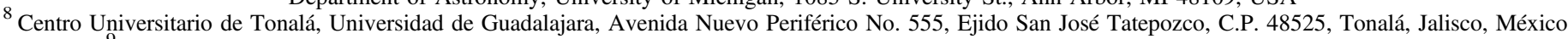 \\ ${ }^{9}$ Division of Physics, Math and Astronomy, California Institute of Technology, 1200 East California Boulevard, Pasadena, CA 91125, USA \\ ${ }^{10}$ Department of Astronomy, The University of Texas at Austin, 2515 Speedway, Stop C1400, Austin, TX 78712-1205, USA \\ ${ }^{11}$ Korea Astronomy and Space Science Institute, 776 Daedeokdaero, Daejeon 305-348, Republic of Korea \\ ${ }^{12}$ Humanitas College, Global Campus, Kyung Hee University, Yongin-shi 17104, Republic of Korea \\ ${ }^{13}$ Cerro Tololo Interamerican Observatory, Casilla 603, La Serena, Chile \\ ${ }^{14}$ Homer L. Dodge Department of Physics and Astronomy, University of Oklahoma, 440 W. Brooks Street, Norman, OK 73019, USA \\ ${ }^{15}$ Department of Astronomy, University of Massachusetts, Amherst, MA 01003, USA \\ Received 2018 February 9; revised 2018 March 29; accepted 2018 April 15; published 2018 May 21
}

\begin{abstract}
We present new trigonometric parallaxes and proper motions of young stellar objects in the Taurus molecular cloud complex from observations collected with the Very Long Baseline Array as part of the Gould's Belt Distances Survey. We detected 26 young stellar objects and derived trigonometric parallaxes for 18 stars with an accuracy of $0.3 \%$ to a few percent. We modeled the orbits of six binaries and determined the dynamical masses of the individual components in four of these systems (V1023 Tau, T Tau S, V807 Tau, and V1000 Tau). Our results are consistent with the first trigonometric parallaxes delivered by the Gaia satellite and reveal the existence of significant depth effects. We find that the central portion of the dark cloud Lynds 1495 is located at $d=$ $129.5 \pm 0.3 \mathrm{pc}$, while the $\mathrm{B} 216$ clump in the filamentary structure connected to it is at $d=158.1 \pm 1.2 \mathrm{pc}$. The closest and remotest stars in our sample are located at $d=126.6 \pm 1.7 \mathrm{pc}$ and $d=162.7 \pm 0.8 \mathrm{pc}$, yielding a distance difference of about $36 \mathrm{pc}$. We also provide a new distance estimate for HL Tau that was recently imaged. Finally, we compute the spatial velocity of the stars with published radial velocity and investigate the kinematic properties of the various clouds and gas structures in this region.
\end{abstract}

Key words: astrometry - binaries: visual - radiation mechanisms: non-thermal - stars: distances - stars: kinematics and dynamics - techniques: interferometric

Supporting material: machine-readable tables

\section{Introduction}

Since the discovery of the first T Tauri star (Joy 1945), the nearby Taurus-Auriga molecular cloud complex (or simply "Taurus") has become one of the most studied regions of lowmass star formation (see, e.g., Kenyon et al. 2008, for the most recent review). Taurus hosts more than 300 known young stellar objects (YSOs), including pre-main-sequence stars and brown dwarfs that are spread over several star-forming clouds and clumps (Luhman et al. 2009; Joncour et al. 2017).

Taurus is composed of multiple filaments (Schneider \& Elmegreen 1979; Hartmann 2002; Schmalzl et al. 2010; Panopoulou et al. 2014), and the spatial distribution of the YSOs in the plane of the sky shows that they are clustered in small groups in or around the different star-forming clouds (Gomez et al. 1993). The question then arises whether the various groups and substructures are bound and have a common origin. Although the morphology of the molecular clouds has been well characterized in previous studies based, e.g., on $\mathrm{CO}$ surveys and extinction maps (Ungerechts \&
Thaddeus 1987; Cambrésy 1999; Dame et al. 2001; Dobashi et al. 2005), little progress has been made so far to constrain the three-dimensional structure of the complex. Distances to individual stars are urgently required to accurately determine the most fundamental properties of YSOs (luminosity, mass, and age), and they could also provide important clues to unravel the history of star formation in this region.

The distance to Taurus is commonly accepted to be $140 \mathrm{pc}$ (Elias 1978) based on several estimates using a wide variety of techniques. The first results obtained by Greenstein \& Shapley (1937) and McCuskey (1939) using star counts returned a distance of 145 and $142 \mathrm{pc}$, respectively. Racine (1968) determined a shorter distance of $135 \pm 10 \mathrm{pc}$ from the photometry of bright stars associated with reflection nebulae. On the other hand, Gottlieb \& Upson (1969) obtained a somewhat larger distance of $150 \mathrm{pc}$ based on the reddening turn-on method. Later, Straizys \& Meistas (1980) investigated the area around the dark clouds L1538, L1528, L1521, and L1495 from the Lynds catalog (Lynds 1962) using a similar approach and concluded that they extend from 140 to $175 \mathrm{pc}$. 
In a companion study, Meistas \& Straizys (1981) found that the front edge of the southern clouds in the complex (L1551, L1546, and L1543) seems to be located at about $140 \mathrm{pc}$. More recently, Kenyon et al. (1994) derived the canonical distance of $140 \pm 10 \mathrm{pc}$ for the northern portion of the Taurus clouds based on the method of spectroscopic parallaxes that is commonly used in the literature. The latter result confirmed previous distance estimates to the Taurus region and supported the idea of a common distance to the various clouds of the complex.

It is important to note that the measurements listed here refer to the mean distance to Taurus based on indirect methods, while the individual distances to most YSOs still remain poorly constrained. Trigonometric parallaxes in the Hipparcos catalog (ESA 1997) exist only for 17 stars with a median error of about 27\%. Bertout et al. (1999) divided them into three groups (L1495 region, Auriga region, and south Taurus) and calculated the mean distance. Using only single stars, they concluded that the three subgroups are located, respectively, at $125_{-16}^{+21}, 140_{-13}^{+16}$, and $168_{-28}^{+42} \mathrm{pc}$. These values are statistically compatible between themselves, but they suggest important distance differences among the various clouds in the complex. The mean parallax of all (single) stars together yields a distance of $139_{-9}^{+10} \mathrm{pc}$, which confirms previous measurements but at the same time blurs the existence of possible depth effects in this region.

In a different study, Bertout \& Genova (2006) applied a variant of the convergent point method and derived the kinematic parallaxes for 67 cluster members using proper motions and radial velocities. The derived parallaxes have a typical error of $20 \%$. They investigated the distances of the YSO subclasses in their sample and concluded that the classical $\mathrm{T}$ Tauri stars are at distances between 126 and $173 \mathrm{pc}$, while the weak emission line T Tauri stars can be found on both sides of the molecular clouds between 106 and 259 pc. These results are indicative of real distance differences among Taurus stars, which will be tested when more trigonometric parallaxes become available.

Very Long Baseline Interferometry (VLBI) has been used in recent years to deliver trigonometric parallaxes of nearby stars with an accuracy often better than $1 \%$ (see, e.g., Melis et al. 2014; Forbrich et al. 2016). The first VLBI trigonometric parallax in Taurus was reported by Lestrade et al. (1999), who targeted the weak-line T Tauri star V773 Tau, which was found to be at $148 \pm 5 \mathrm{pc}$. Later, Torres et al. (2012) presented an improved solution for its distance, which takes into account the orbital motion of the binary system, placing it at $132.8 \pm 2.3 \mathrm{pc}$. The second trigonometric parallax obtained from VLBI radio observations in Taurus was obtained by Loinard et al. (2007). They measured the distance of $147.6 \pm 0.6 \mathrm{pc}$ for T Tau Sb in the well-known T Tau triple system in the southern clouds of the complex. Subsequently, Torres et al. (2007) measured the trigonometric parallaxes of Hubble 4 and HDE 283572 in the central portion of the complex and derived the distances of $132.8 \pm 0.5 \mathrm{pc}$ and $128.5 \pm 0.6 \mathrm{pc}$, respectively. In a companion study, Torres et al. (2009) measured a somewhat larger distance of $161.2 \pm 0.9 \mathrm{pc}$ for HP Tau G2, making it the most distant star with known trigonometric parallax in Taurus. In summary, V773 Tau, Hubble 4, and HDE 283572, which are associated with the most prominent dark cloud L1495, are found to be at the same distance, while T Tau Sb and HP Tau G2 are located at larger distances. There is growing evidence of significant dispersion along the line of sight, but the small number of cluster members with measured trigonometric parallaxes is still insufficient to construct a precise three-dimensional map of this region.

This paper is one in a series dedicated to measuring stellar distances based on VLBI observations as part of the Gould's Belt Distances Survey (GOBELINS; Loinard et al. 2011). Previous papers of this project investigated the Ophiuchus (Ortiz-León et al. 2017b), Orion (Kounkel et al. 2017), and Serpens (Ortiz-León et al. 2017a) star-forming regions. Here, we present new trigonometric parallaxes and proper motions for YSOs in Taurus. It is organized as follows. In Section 2 we describe our sample and observations. The methodology used to fit the astrometry for both single stars and binaries is explained in Section 3. In Section 4 we present our results and comment on individual targets. In Section 5 we compare our results derived in this work with the trigonometric parallaxes delivered by the first data release of the Gaia space mission (Gaia-DR1; Gaia Collaboration et al. 2016) for the targets in common, and we complement our sample with the Gaia stars not included in our observing program to construct the most complete, precise, and accurate picture of three-dimensional structure of the Taurus region to date. In this section, we also discuss the kinematic properties of the stars and molecular clouds in this region (see Table 10). Finally, we summarize our results and conclusions in Section 6.

\section{Sample and Observations}

In a recent study, Dzib et al. (2015) reported on multiepoch radio observations of the Taurus complex using the Karl G. Jansky Very Large Array (VLA). They detected 59 sources related to YSOs, 18 field stars, and another 46 unidentified sources whose radio properties are consistent with YSOs. However, only $56 \%$ of the young stars identified in their study exhibit properties compatible with nonthermal radio emission that can be detected with VLBI observations. These sources constitute the starting point of our sample for the GOBELINS project in the Taurus region.

The observations presented in this paper were obtained with the Very Long Baseline Array (VLBA) near the equinoxes of every year between 2012 August and 2017 October. The data were recorded in dual polarization mode with a bandwidth of $256 \mathrm{MHz}$ centered at $\nu=5.0$ or $8.4 \mathrm{GHz}(\mathrm{C}$ and $\mathrm{X}$ bands, respectively). We observed with the $\mathrm{X}$ band during the first $3 \mathrm{yr}$ of our observing program and then switched to the $\mathrm{C}$ band, which reduced significantly the noise in our observations. The VLBA was pointed at the position of our targets that have been accommodated in 52 different fields. Table 1 lists the observed epochs, bands, pointing positions, and calibrators for each field. In some of these fields two or more targets are observed simultaneously. We included additional phase centers within the primary beam to observe other sources reported by Dzib et al. (2015) independently of their nature (YSO candidates, field stars, or extragalactic sources) as part of our observing strategy (see Ortiz-León et al. 2017b, for more details). Doing so, we observed a total of 86 sources (or stellar systems) during our observing campaign, of which 45 are known YSOs in the literature. As shown in Figure 1, our targets are spread over the various molecular clouds in Taurus.

Our observations produced 164 different projects under the code BL175 (see Table 1). Each observing session consisted of 


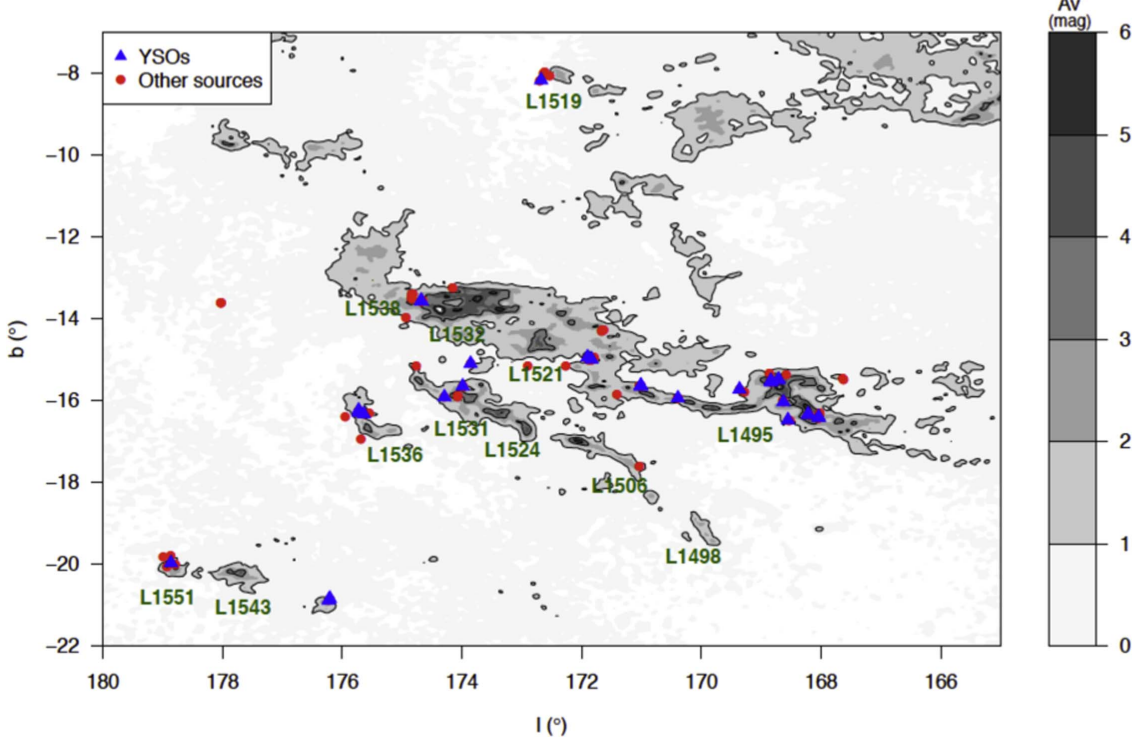

Figure 1. Location of our targets in the Taurus star-forming complex overlaid on the extinction map from Dobashi et al. (2005) in Galactic coordinates. The most prominent Lynds dark clouds (Lynds 1962) in this region are identified in this diagram.

Table 1

Observed Fields in Taurus with the VLBA

\begin{tabular}{lccccc}
\hline \hline Project Code & Date & Band & $\begin{array}{c}\alpha \\
(\mathrm{h}: \mathrm{m}: \mathrm{s})\end{array}$ & $\begin{array}{c}\delta \\
\left({ }^{\prime \prime \prime}\right)\end{array}$ & Calibrators \\
\hline BL175C1 & 2012 Aug 20 & $\mathrm{X}$ & 042202.20 & 193327.0 & $\mathrm{~J} 0412+1856, \mathrm{~J} 0428+1732, \mathrm{~J} 0426+2327, \mathrm{~J} 0412+2305$ \\
BL175C2 & 2012 Aug 30 & $\mathrm{X}$ & 043125.13 & 181616.6 & $\mathrm{~J} 0431+1731, \mathrm{~J} 0428+1732, \mathrm{~J} 0438+2153, \mathrm{~J} 0440+1437$ \\
BL175C3 & 2012 Aug 31 & $\mathrm{X}$ & 043140.09 & 181356.7 & $\mathrm{~J} 0431+1731, \mathrm{~J} 0428+1732, \mathrm{~J} 0438+2153, \mathrm{~J} 0440+1437$ \\
BL175C4 & 2012 Sep 10 & $\mathrm{X}$ & 043214.58 & 182014.6 & $\mathrm{~J} 0431+1731, \mathrm{~J} 0428+1732, \mathrm{~J} 0438+2153, \mathrm{~J} 0440+1437$ \\
BL175C5 & 2012 Sep 30 & $\mathrm{X}$ & 043134.15 & 180804.6 & $\mathrm{~J} 0431+1731, \mathrm{~J} 0428+1732, \mathrm{~J} 0438+2153, \mathrm{~J} 0440+1437$ \\
& & & 043229.47 & 181400.3 & \\
BL175C6 & 2012 Nov 30 & $\mathrm{X}$ & 043326.35 & 222832.0 & $\mathrm{~J} 0438+2153, \mathrm{~J} 0450+2249, \mathrm{~J} 0426+2350, \mathrm{~J} 0431+1731$ \\
BL175C7 & 2012 Dec 02 & $\mathrm{X}$ & 043513.27 & 225920.0 & $\mathrm{~J} 0438+2153, \mathrm{~J} 0426+2327, \mathrm{~J} 0435+2532, \mathrm{~J} 0450+2249$ \\
BL175C8 & 2012 Dec 03 & $\mathrm{X}$ & 043520.91 & 225424.0 & $\mathrm{~J} 0438+2153, \mathrm{~J} 0426+2327, \mathrm{~J} 0435+2532, \mathrm{~J} 0450+2249$ \\
BL175C9 & 2012 Dec 04 & $\mathrm{X}$ & 043548.11 & 225329.1 & $\mathrm{~J} 0438+2153, \mathrm{~J} 0426+2327, \mathrm{~J} 0435+2532, \mathrm{~J} 0450+2249$ \\
\hline
\end{tabular}

Note. We provide for each field the NRAO project code, epoch of observation, observed band, coordinates of the field center, and the calibrators (the main calibrator is the first source in the list).

(This table is available in its entirety in machine-readable form.)

cycles alternating between the target(s) and the main phase calibrator. The secondary calibrators were observed every $\sim 50$ minutes. In addition, we also observed blocks of geodetic calibrators over a wide range of elevations at the beginning and end of each session. The typical integration time in each cycle was $\sim 2$ minutes for science targets and $\sim 1$ minute for calibrators. The total integration time for projects that observed with the $\mathrm{X}$ band and $\mathrm{C}$ band were $1.1 \mathrm{and} 1.5 \mathrm{hr}$, respectively. The calibrators used in our observations are extragalactic sources, and the positions of all sources in each project are referenced to the corresponding primary calibrator. The typical angular separation between the primary calibrator and the YSOs in our sample ranges from 0.9 to 3.7 .

The VLBA observations were correlated with the DiFX software correlator (Deller et al. 2011). Then, the data were edited and calibrated using the Astronomical Image Processing System (AIPS; Greisen 2003) following the standard prescription for VLBA data as described in Ortiz-León et al. (2017b). We applied the same calibration to all sources in the field when multiple targets were observed in the same session. The calibrated visibilities were imaged with a pixel size of $50-100 \mu$ as and resulted in a typical angular resolution of 3 mas $\times 1$ mas at $\nu=5.0 \mathrm{GHz}$ and 2 mas $\times 0.8$ mas at $\nu=$ $8.4 \mathrm{GHz}$. The mean noise level in the calibrated images was 26 and $42 \mu \mathrm{Jy}$ beam $^{-1}$ at the $\mathrm{C}$ and X bands, respectively. Finally, the source position (and the corresponding errors) were obtained from a two-dimensional Gaussian fitting using the AIPS task JMFIT.

As mentioned before, we observed a total of 86 targets in Taurus for the GOBELINS project, but only 52 sources could be detected in our observations. Table 2 lists the sources that have been observed in our campaign with the VLBA. We provide the minimum and maximum flux density measured at both frequencies, the brightness temperature $T_{\mathrm{B}}$, the number of detections, and observations for each source. In some cases we provide an upper flux density limit of $3 \sigma$ (based on the noise level of the image) when the source was observed but could not be detected. In this context, it is important to mention that some of the YSOs targeted in this study are highly variable. For binaries and multiple systems that could be resolved in our 
Table 2

VLBA Detections and Nondetections in Taurus

\begin{tabular}{|c|c|c|c|c|c|c|c|c|}
\hline $\begin{array}{l}\text { GBS-VLA }^{a} \\
\text { Identifier } \\
\text { (1) }\end{array}$ & $\begin{array}{l}\text { Other } \\
\text { Identifier } \\
\text { (2) }\end{array}$ & $\begin{array}{l}\text { Minimum Flux } \\
\text { at } 5.0 \mathrm{GHz}(\mathrm{mJy}) \\
(3)\end{array}$ & $\begin{array}{c}\text { Maximum Flux } \\
\text { at } 5.0 \mathrm{GHz}(\mathrm{mJy}) \\
(4)\end{array}$ & $\begin{array}{l}\text { Minimum Flux } \\
\text { at } 8.4 \mathrm{GHz}(\mathrm{mJy}) \\
(5)\end{array}$ & $\begin{array}{c}\text { Maximum Flux } \\
\text { at } 8.4 \mathrm{GHz}(\mathrm{mJy}) \\
(6)\end{array}$ & $\begin{array}{c}\log T_{\mathrm{B}} \\
\text { (7) }\end{array}$ & $\begin{array}{c}\text { Type }^{\text {b }} \\
(8)\end{array}$ & $\begin{array}{l}\text { Number } \\
\text { Det./Obs. } \\
\text { (9) }\end{array}$ \\
\hline J041327.23+281624.4 & V1096 Tau A & $0.71 \pm 0.05$ & $9.2 \pm 0.07$ & $0.38 \pm 0.08$ & $0.55 \pm 0.08$ & 8.03 & YSO & $4 / 8$ \\
\hline J041327.23+281624.4 & V1096 Tau B & $0.20 \pm 0.04$ & $0.36 \pm 0.05$ & & & 6.75 & YSO & $3 / 8$ \\
\hline J041412.93+281211.9 & V773 Tau Aa & $2.77 \pm 0.07$ & $11.89 \pm 0.07$ & $0.36 \pm 0.08$ & $22.23 \pm 0.20$ & 8.66 & YSO & $7 / 7$ \\
\hline J041412.93+281211.9 & V773 Tau Ab & \multicolumn{2}{|c|}{$1.56 \pm 0.07$} & $0.54 \pm 0.11$ & $1.57 \pm 0.10$ & 7.45 & YSO & $3 / 7$ \\
\hline J041448.00+275234.4 & V1098 Tau & $0.22 \pm 0.06$ & $0.29 \pm 0.06$ & \multicolumn{2}{|c|}{$1.41 \pm 0.1$} & 7.48 & YSO & $3 / 8$ \\
\hline J041628.11+280735.4 & V1068 Tau & \multicolumn{2}{|c|}{$11.19 \pm 0.09$} & \multicolumn{2}{|c|}{$0.40 \pm 0.13$} & 8.34 & YSO & $2 / 7$ \\
\hline J041829.10+282618.8 & 2MASS J04182909+2826191 & $0.54 \pm 0.05$ & $1.79 \pm 0.04$ & \multicolumn{2}{|c|}{$1.31 \pm 0.1$} & 7.47 & YSO & $3 / 9$ \\
\hline J041831.12+282715.9 & HD 283518, V410 Tau & $1.21 \pm 0.04$ & $3.59 \pm 0.06$ & $0.72 \pm 0.09$ & $13.62 \pm 0.22$ & 8.44 & YSO & $9 / 9$ \\
\hline J041840.62+281915.3 & V892 Tau & \multicolumn{2}{|c|}{$0.22 \pm 0.06$} & $0.33 \pm 0.10$ & $0.38 \pm 0.11$ & 6.60 & YSO & $3 / 14$ \\
\hline J041847.04+282007.2 & Hubble 4a, V1023 Tau A & $0.63 \pm 0.06$ & $1.44 \pm 0.04$ & $0.48 \pm 0.09$ & $14.94 \pm 0.44$ & 8.53 & YSO & $14 / 14$ \\
\hline J041847.04+282007.2 & Hubble 4b, V1023 Tau B & $0.52 \pm 0.04$ & $0.52 \pm 0.05$ & \multicolumn{2}{|c|}{$\ldots$} & 6.95 & YSO & $2 / 14$ \\
\hline J041941.28+274947.9 & V1070 Tau & \multicolumn{2}{|c|}{$0.29 \pm 0.06$} & \multicolumn{2}{|c|}{$0.58 \pm 0.10$} & 7.02 & YSO & $2 / 7$ \\
\hline J042159.43+193205.7 & T Tau Sb & $2.06 \pm 0.06$ & $2.62 \pm 0.07$ & $1.58 \pm 0.09$ & $4.58 \pm 0.09$ & 7.65 & YSO & $8 / 8$ \\
\hline J042200.70+265732.2 & 2MASS J04220069+2657324 & \multicolumn{2}{|c|}{$0.15 \pm 0.05$} & \multicolumn{2}{|c|}{$<0.14$} & 6.15 & YSO & $1 / 7$ \\
\hline J042204.97+193448.3 & 2MASS J04220496+1934483 & \multicolumn{2}{|c|}{$0.19 \pm 0.05$} & \multicolumn{2}{|c|}{$<0.11$} & 6.48 & YSO & $1 / 8$ \\
\hline J042448.16+264315.9 & V1201 Tau A & $0.15 \pm 0.05$ & $2.40 \pm 0.04$ & \multicolumn{2}{|c|}{$\ldots$} & 7.64 & YSO & $2 / 8$ \\
\hline J042448.16+264315.9 & V1201 Tau B & $0.13 \pm 0.04$ & $0.46 \pm 0.06$ & $0.52 \pm 0.07$ & $0.53 \pm 0.06$ & 7.00 & YSO & $6 / 8$ \\
\hline $\mathrm{J} 042449.05+264310.2$ & HD 283641 & $0.36 \pm 0.07$ & $1.08 \pm 0.06$ & $0.45 \pm 0.08$ & $0.91 \pm 0.09$ & 7.26 & YSO & $5 / 6$ \\
\hline J042920.70+263340.2 & 2MASS J04292071+2633406 & \multirow{2}{*}{\multicolumn{2}{|c|}{$\begin{array}{c}\cdots \\
0.46 \pm 0.05\end{array}$}} & \multicolumn{2}{|c|}{$0.22 \pm 0.07$} & 6.65 & YSO & $1 / 4$ \\
\hline J042942.47+263249.0 & DI Tau & & & \multicolumn{2}{|c|}{$<0.11$} & 6.73 & YSO & $1 / 9$ \\
\hline J043140.09+181356.7 & XZ Tau & $0.18 \pm 0.05$ & $0.70 \pm 0.05$ & \multicolumn{2}{|c|}{$0.37 \pm 0.1$} & 7.06 & YSO & $5 / 9$ \\
\hline J043306.62+240954.8 & V807 Tau Ba & $0.28 \pm 0.05$ & $1.83 \pm 0.05$ & \multicolumn{2}{|c|}{$0.36 \pm 0.07$} & 7.51 & YSO & $5 / 9$ \\
\hline J043310.04+243343.1 & V830 Tau & \multicolumn{2}{|c|}{$1.02 \pm 0.06$} & \multicolumn{2}{|c|}{$0.5 \pm 0.08$} & 7.18 & YSO & $2 / 9$ \\
\hline J043439.24+250100.9 & V1110 Tau & \multicolumn{2}{|c|}{$0.64 \pm 0.06$} & $0.39 \pm 0.08$ & $0.57 \pm 0.07$ & 7.07 & YSO & $4 / 7$ \\
\hline J043520.92+225424.0 & FF Tau & \multicolumn{2}{|c|}{$0.22 \pm 0.05$} & & & 6.53 & YSO & $1 / 9$ \\
\hline J043542.05+225222.5 & 2MASS J04354203+2252226 & 0.2 & & & & 7.20 & YSO & $2 / 9$ \\
\hline J043554.17+225413.3 & HP Tau G2 & $0.66 \pm 0.05$ & $4.55 \pm 0.05$ & $0.46 \pm 0.08$ & $3.07 \pm 0.08$ & 7.90 & YSO & $9 / 9$ \\
\hline $\mathrm{J} 044205.49+252256.0$ & V999 Tau & $0.15 \pm 0.04$ & $0.83 \pm 0.05$ & & & 7.17 & YSO & $5 / 12$ \\
\hline J044207.32+252303.0 & V1000 Tau A & $0.10 \pm 0.04$ & $0.96 \pm 0.05$ & $0.22 \pm 0.08$ & $0.29 \pm 0.09$ & 7.18 & YSO & $8 / 11$ \\
\hline J044207.32+252303.0 & V1000 Tau B & $0.11 \pm 0.04$ & $0.22 \pm 0.05$ & $0.19 \pm 0.07$ & $0.22 \pm 0.08$ & 6.59 & YSO & $6 / 11$ \\
\hline J045536.97+301754.8 & HD 282630 & $0.28 \pm 0.05$ & $0.37 \pm 0.04$ & & .08 & 6.85 & YSO & $3 / 7$ \\
\hline J042922.26+263728.6 & SDSS J042922.24+263728.7 & 0.1 & & & & 6.62 & Star? & $2 / 8$ \\
\hline J042926.77+263047.8 & & & & & & 6.57 & Star? & $1 / 7$ \\
\hline J043113.01+270834.8 & WISE J043113.00+270834.9 & & & & & 6.59 & Star? & $1 / 6$ \\
\hline J041443.21+275347.5 & & $1.13 \pm 0.05$ & $1.14 \pm 0.05$ & $0.61 \pm 0.08$ & $0.76 \pm 0.08$ & 7.31 & $\mathrm{~B}$ & $6 / 6$ \\
\hline J041515.93+291244.5 & WISE J041515.85+291244.3 & & & $0.23 \pm 0.06$ & $0.54 \pm 0.09$ & 7.06 & $\mathrm{~B}$ & $5 / 5$ \\
\hline J041825.42+252156.4 & WISE J041825.43+252156.4 & 0.3 & & $0.31 \pm 0.07$ & $0.37 \pm 0.08$ & 6.82 & $\mathrm{~B}$ & $3 / 4$ \\
\hline J042449.45+264304.1 & & $0.19 \pm 0.05$ & $0.24 \pm 0.04$ & & & 6.80 & $\mathrm{~B}$ & $3 / 6$ \\
\hline J042452.48+264204.5 & SDSS J042452.47+264204.5 & & & $3.87 \pm 0.07$ & $4.48 \pm 0.09$ & 7.95 & $\mathrm{~B}$ & $4 / 4$ \\
\hline J042920.74+263353.4 & JH 507 & & & $0.19 \pm 0.05$ & $0.55 \pm 0.07$ & 7.04 & $\mathrm{~B}$ & $4 / 4$ \\
\hline J042929.49+263152.8 & 2MASS J04292949+2631528 & & & $13.33 \pm 0.13$ & $17.62 \pm 0.10$ & 8.62 & $\mathrm{~B}$ & $5 / 5$ \\
\hline J042939.59+263110.7 & & & & $0.16 \pm 0.05$ & $0.29 \pm 0.08$ & 6.75 & $\mathrm{~B}$ & $2 / 5$ \\
\hline J043109.20+271045.3 & & & & $0.23 \pm 0.07$ & $0.47 \pm 0.11$ & 6.81 & $\mathrm{~B}$ & $4 / 4$ \\
\hline
\end{tabular}


Table 2

(Continued)

\begin{tabular}{|c|c|c|c|c|c|c|c|c|}
\hline $\begin{array}{l}\text { GBS-VLA }^{a} \\
\text { Identifier } \\
\text { (1) }\end{array}$ & $\begin{array}{l}\text { Other } \\
\text { Identifier } \\
(2)\end{array}$ & $\begin{array}{l}\text { Minimum Flux } \\
\text { at } 5.0 \mathrm{GHz}(\mathrm{mJy}) \\
(3)\end{array}$ & $\begin{array}{c}\text { Maximum Flux } \\
\text { at } 5.0 \mathrm{GHz}(\mathrm{mJy}) \\
(4)\end{array}$ & $\begin{array}{c}\text { Minimum Flux } \\
\text { at } 8.4 \mathrm{GHz}(\mathrm{mJy}) \\
(5)\end{array}$ & $\begin{array}{c}\text { Maximum Flux } \\
\text { at } 8.4 \mathrm{GHz}(\mathrm{mJy}) \\
(6)\end{array}$ & $\begin{array}{c}\log T_{\mathrm{B}} \\
(7)\end{array}$ & $\begin{array}{c}\text { Type }^{b} \\
(8)\end{array}$ & $\begin{array}{c}\text { Number } \\
\text { Det./Obs. } \\
(9)\end{array}$ \\
\hline J043229.46+181400.3 & 2MASS J04322946+1814002 & \multicolumn{2}{|c|}{$\ldots$} & $7.84 \pm 0.36$ & $68.91 \pm 0.64$ & 9.09 & B & $5 / 5$ \\
\hline J043235.22+242021.4 & XEST 03-026 & \multicolumn{2}{|c|}{$\cdots$} & $20.39 \pm 0.13$ & $27.71 \pm 0.18$ & 8.77 & $\mathrm{~B}$ & $4 / 4$ \\
\hline J043237.91+242054.5 & & \multicolumn{2}{|c|}{$0.37 \pm 0.05$} & $0.34 \pm 0.09$ & $0.64 \pm 0.09$ & 7.17 & $\mathrm{~B}$ & $5 / 5$ \\
\hline J043306.02+243254.4 & WISE J043305.95+243253.8 & $0.16 \pm 0.05$ & $0.20 \pm 0.05$ & \multicolumn{2}{|c|}{$0.22 \pm 0.07$} & 6.64 & $\mathrm{~B}$ & $3 / 6$ \\
\hline J043326.35+222832.0 & SDSS J043326.34+222831.9 & & & $0.55 \pm 0.06$ & $1.08 \pm 0.08$ & 7.33 & $\mathrm{~B}$ & $4 / 4$ \\
\hline J044144.92+255815.3 & WISE J044144.86+255815.0 & $0.17 \pm 0.05$ & $0.19 \pm 0.06$ & $0.16 \pm 0.06$ & $0.18 \pm 0.06$ & 6.51 & $\mathrm{~B}$ & $4 / 6$ \\
\hline J044210.59+252505.4 & WISE J044210.55+252505.6 & $0.27 \pm 0.07$ & $0.43 \pm 0.06$ & $0.28 \pm 0.06$ & $0.30 \pm 0.08$ & 7.13 & B & $7 / 12$ \\
\hline J044210.79+252420.2 & WISE J044210.55+252505.6 & \multicolumn{2}{|c|}{$\cdots$} & $0.94 \pm 0.12$ & $1.81 \pm 0.10$ & 7.58 & $\mathrm{~B}$ & $8 / 8$ \\
\hline $\mathrm{J} 044246.20+251806.2$ & & \multicolumn{2}{|c|}{$\ldots$} & $0.17 \pm 0.05$ & $0.27 \pm 0.06$ & 6.72 & $\mathrm{~B}$ & $2 / 4$ \\
\hline J044247.78+251825.7 & WISE J044247.80+251825.3 & \multicolumn{2}{|c|}{$\cdots$} & $0.67 \pm 0.07$ & $0.76 \pm 0.09$ & 7.17 & $\mathrm{~B}$ & $4 / 4$ \\
\hline J044252.01+251904.9 & WISE J044251.97+251905.1 & \multicolumn{2}{|c|}{$\ldots$} & $3.61 \pm 0.14$ & $4.26 \pm 0.12$ & 7.91 & $\mathrm{~B}$ & $4 / 4$ \\
\hline J044308.05+252210.2 & 2MASS J04430807+2522103 & \multicolumn{2}{|c|}{$\cdots$} & $3.20 \pm 0.09$ & $4.15 \pm 0.08$ & 7.90 & $\mathrm{~B}$ & $4 / 4$ \\
\hline J045539.28+301627.2 & WISE J045539.26+301627.1 & \multicolumn{2}{|c|}{$\cdots$} & $0.72 \pm 0.06$ & $1.00 \pm 0.08$ & 7.23 & $\mathrm{~B}$ & $4 / 4$ \\
\hline $\mathrm{J} 045607.08+302726.7$ & & \multicolumn{2}{|c|}{$\cdots$} & $0.22 \pm 0.06$ & $0.47 \pm 0.09$ & 6.94 & B & $4 / 4$ \\
\hline J041343.94+282054.8 & & \multicolumn{2}{|c|}{$\cdots$} & \multicolumn{2}{|c|}{$<0.12$} & $\cdots$ & $\cdots$ & $0 / 4$ \\
\hline J041343.94+282054.8 & & \multicolumn{2}{|c|}{$\ldots$} & \multicolumn{2}{|c|}{$<0.12$} & $\cdots$ & $\cdots$ & $0 / 4$ \\
\hline J041412.58+281155.7 & 2MASS J04141256+2811561 & \multicolumn{2}{|c|}{$\cdots$} & \multicolumn{2}{|c|}{$<0.14$} & $\cdots$ & $\cdots$ & $0 / 4$ \\
\hline J041519.37+291247.9 & WISE J041519.37+291247.7 & \multicolumn{2}{|c|}{$<0.06$} & \multicolumn{2}{|c|}{$<0.10$} & $\cdots$ & $\cdots$ & $0 / 7$ \\
\hline J041635.09+280818.8 & & \multicolumn{2}{|c|}{$\ldots$} & \multicolumn{2}{|c|}{$<0.10$} & $\cdots$ & $\cdots$ & $0 / 4$ \\
\hline $\mathrm{J} 041833.38+283732.2$ & 2MASS J04183343+2837321 & \multicolumn{2}{|c|}{$<0.08$} & \multicolumn{2}{|c|}{$<0.13$} & $\cdots$ & YSO & $0 / 6$ \\
\hline $\mathrm{J} 041840.27+282036.0$ & & & & & & $\cdots$ & $\cdots$ & $0 / 14$ \\
\hline J041851.49+282026.1 & 2MASS J04185147+2820264 & & & & & $\cdots$ & YSO & $0 / 9$ \\
\hline J041916.12+275048.2 & 2MASS J04191612+2750481 & & & & & $\cdots$ & $\cdots$ & $0 / 5$ \\
\hline J041926.27+282614.0 & V819 Tau & & & & & $\cdots$ & YSO & $0 / 5$ \\
\hline J041940.54+274953.6 & SDSS J041940.55+274953.5 & & & & & $\cdots$ & $\cdots$ & $0 / 4$ \\
\hline $\mathrm{J} 042202.20+265730.3$ & FS Tau & & & & & $\cdots$ & YSO & $0 / 6$ \\
\hline J042202.63+265709.1 & & & & & & $\cdots$ & $\cdots$ & $0 / 4$ \\
\hline J042517.49+261748.4 & SDSS J042517.49+261748.5 & & & & & $\cdots$ & $\cdots$ & $0 / 5$ \\
\hline J042939.80+263222.9 & & & & & & $\cdots$ & $\cdots$ & $0 / 5$ \\
\hline J043002.25+260843.4 & SDSS J043002.24+260843.4 & & & & & $\cdots$ & $\cdots$ & $0 / 4$ \\
\hline J043114.45+271017.6 & V1320 Tau & & & & & $\cdots$ & YSO & $0 / 4$ \\
\hline J043116.56+271006.9 & & & & & & $\cdots$ & $\cdots$ & $0 / 4$ \\
\hline J043125.13+181616.6 & V1073 Tau, HD 285845 & & & & & $\cdots$ & $\cdots$ & $0 / 5$ \\
\hline J043134.15+180804.6 & LDN 1551 IRS 5B & & & & & $\cdots$ & YSO & $0 / 5$ \\
\hline J043148.73+254021.8 & SDSS J043148.70+254021.6 & & & & & $\cdots$ & $\cdots$ & $0 / 4$ \\
\hline J043214.58+182014.6 & V827 Tau & & & & & $\cdots$ & YSO & $0 / 5$ \\
\hline J043235.57+242008.5 & & & & & & $\cdots$ & $\cdots$ & $0 / 4$ \\
\hline J043513.27+225920.2 & EZ Tau & & & & & $\cdots$ & $\cdots$ & $0 / 7$ \\
\hline J043550.97+225339.3 & & & & & & $\cdots$ & $\cdots$ & $0 / 7$ \\
\hline J043553.52+225408.9 & HP Tau G3 & & & & & $\cdots$ & YSO & $0 / 5$ \\
\hline J043558.97+223835.2 & 2MASS J04355892+2238353 & & & & & $\cdots$ & YSO & $0 / 4$ \\
\hline J043657.44+241835.1 & & & & & & $\cdots$ & $\cdots$ & $0 / 3$ \\
\hline J044123.47+245528.1 & & & & & & $\cdots$ & $\cdots$ & $0 / 4$ \\
\hline
\end{tabular}


Table 2

(Continued)

\begin{tabular}{|c|c|c|c|c|c|c|c|c|}
\hline $\begin{array}{l}\text { GBS-VLA }{ }^{a} \\
\text { Identifier } \\
\text { (1) }\end{array}$ & $\begin{array}{l}\text { Other } \\
\text { Identifier } \\
\text { (2) }\end{array}$ & $\begin{array}{l}\text { Minimum Flux } \\
\text { at } 5.0 \mathrm{GHz}(\mathrm{mJy}) \\
\text { (3) }\end{array}$ & $\begin{array}{c}\text { Maximum Flux } \\
\text { at } 5.0 \mathrm{GHz}(\mathrm{mJy}) \\
(4)\end{array}$ & $\begin{array}{l}\text { Minimum Flux } \\
\text { at } 8.4 \mathrm{GHz}(\mathrm{mJy}) \\
(5)\end{array}$ & $\begin{array}{l}\text { Maximum Flux } \\
\text { at } 8.4 \mathrm{GHz}(\mathrm{mJy}) \\
(6)\end{array}$ & $\begin{array}{l}\log T_{\mathrm{B}} \\
\text { (7) }\end{array}$ & $\begin{array}{c}\text { Type }^{b} \\
(8)\end{array}$ & $\begin{array}{c}\text { Number } \\
\text { Det./Obs. } \\
\text { (9) }\end{array}$ \\
\hline J044209.56+252427.7 & & & & \multicolumn{2}{|c|}{$<0.11$} & $\ldots$ & $\ldots$ & $0 / 7$ \\
\hline $\mathrm{J} 044307.69+252348.2$ & WISE J044307.66+252347.5 & & & \multicolumn{2}{|c|}{$<0.11$} & $\ldots$ & $\ldots$ & $0 / 4$ \\
\hline J045534.44+302808.3 & XEST 26-028 & & & \multicolumn{2}{|c|}{$<0.11$} & $\ldots$ & $\ldots$ & $0 / 4$ \\
\hline $\mathrm{J} 045605.30+302541.7$ & & & & \multicolumn{2}{|c|}{$<0.10$} & $\ldots$ & $\ldots$ & $0 / 4$ \\
\hline $\mathrm{J} 045607.27+302728.2$ & & & & \multicolumn{2}{|c|}{$<0.10$} & $\ldots$ & $\ldots$ & $0 / 4$ \\
\hline
\end{tabular}

\section{Notes.}

${ }^{\mathrm{a}}$ The GBS-VLA (Gould's Belt Very Large Array Survey) identifier is defined by Dzib et al. (2015).

b Type of source: "YSO" = Young Stellar Object; "Star?" = field star; and "B" = background source (Galactic or extragalactic). 


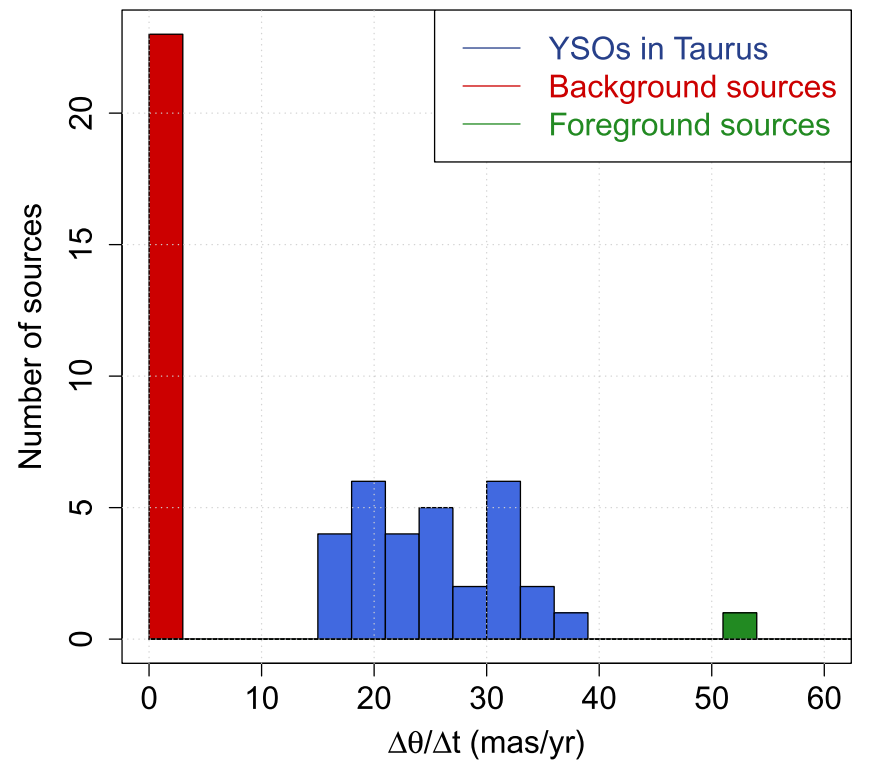

Figure 2. Histogram of the position change rate for all sources (including binaries) observed in our campaign with a minimum of two detections (see Table 2).

observations we present the results for each component separately. We note that most sources in Table 2 exhibit a brightness temperature of $T_{\mathrm{B}}>10^{6} \mathrm{~K}$, which is consistent with nonthermal radio emission. Thirty-four sources in our initial target list were not detected, and we interpret the nondetections with the VLBA as evidence that their radio emission is thermal. We consider a single detection to be valid when the flux density of the source is above a $5 \sigma$ threshold, where $\sigma$ is the corresponding noise level of the image. Our effective sample of detected sources contains 26 stars that have been confirmed as YSOs in previous studies and another three sources that require further monitoring to investigate their membership in the Taurus region. The remaining 23 sources that have been detected in our observations are likely to be background contaminants that are not related to the Taurus molecular clouds. As shown in Figure 2, their proper motions, which are estimated at this stage from the position change rate of the source, are consistent with zero (within the astrometric errors), while the typical proper motion of YSOs in Taurus is about $22 \mathrm{mas} \mathrm{yr}^{-1}$ (see, e.g., Bertout \& Genova 2006).

It is important to mention that some sources in our sample had been observed with the VLBA in the past. Thus, we searched the data archive of the National Radio Astronomy Observatory for additional information on our targets. We collected a total of 65 projects at different epochs that are listed in Table 3. These observations were performed between 2003 September and 2009 March, which allows us to extend the time base of our analysis to more than $10 \mathrm{yr}$ of observations for some targets. This is particularly useful to investigate the orbital motion of binaries and multiple systems in our sample as discussed in the forthcoming sections of this paper. Although the source positions obtained from these observations have already been published in previous studies, we recalibrated these data using the same procedure that was adopted for the GOBELINS project to better combine the different data sets. The typical noise level and angular resolution that we obtain in the recalibrated images from archival data are, respectively, $100 \mu \mathrm{Jy}$ beam $^{-1}$ and 2 mas $\times 0.8$ mas.

\section{Astrometry Fitting}

\subsection{Single Stars}

The displacement of a single star in the plane of the sky is the combination of its proper motion $\left(\mu_{\alpha}, \mu_{\delta}\right)$ and the trigonometric parallax $(\pi)$. In this case, the stellar coordinates as a function of time $(t)$ are given by

$$
\begin{gathered}
\alpha(t)=\alpha_{0}+\mu_{\alpha} t+\pi f_{\alpha}(t), \\
\delta(t)=\delta_{0}+\mu_{\delta} t+\pi f_{\delta}(t),
\end{gathered}
$$

where $\left(\alpha_{0}, \delta_{0}\right)$ are the coordinates of the star at a given epoch $\left(t_{0}\right)$. The projections of the parallactic ellipse $\left(f_{\alpha}, f_{\delta}\right)$ are given by (see, e.g., Seidelmann 1992)

$$
\begin{gathered}
f_{\alpha}=(X \sin \alpha-Y \cos \alpha) / \cos \delta, \\
f_{\delta}=X \cos \alpha \sin \delta+Y \sin \alpha \sin \delta-Z \cos \delta,
\end{gathered}
$$

where $(X, Y, Z)$ are the barycentric coordinates of the Earth (in units of au) computed from the planetary ephemerides DE405 using the novas package in Python.

\subsection{Binaries}

Binaries and multiple systems are very common in the Taurus star-forming region (Duchêne 1999), and our sample contains many such systems that require a dedicated analysis. Since both stars in a binary system move around their common center of gravity, their orbital motion projected onto the plane of the sky has to be taken into account to accurately determine the parallaxes and proper motions of the individual components.

At this stage it is important to distinguish between (i) binaries with orbital periods much longer than the monitoring time of our observing campaign and (ii) binaries with short or intermediate periods where our observations cover a significant fraction of the orbital period. In the first case, it is possible to assume a uniform acceleration (see, e.g., Loinard et al. 2007) leading to

$$
\begin{gathered}
\alpha(t)=\alpha_{0}+\mu_{\alpha} t+\frac{1}{2} a_{\alpha} t^{2}+\pi f_{\alpha}(t), \\
\delta(t)=\delta_{0}+\mu_{\delta} t+\frac{1}{2} a_{\delta} t^{2}+\pi f_{\delta}(t),
\end{gathered}
$$

where $a_{\alpha}$ and $a_{\delta}$ are the acceleration terms. In the second case, the effects of the binary motion and the existence of a nonuniform acceleration need to be taken into account. It is therefore necessary to fit for the full orbital motion and the astrometric parameters simultaneously. The orbital elements to be considered in this case are the semimajor axis $a$, the orbital period $P$, the eccentricity $e$, the epoch of periastron passage $T_{p}$, the argument of the ascending node $\Omega$, the longitude of the periastron $\omega$, and the inclination $i$ of the orbital plane. Thus, the equations for the primary component with semimajor axis $a_{1}$ can be written in the form

$$
\begin{gathered}
\alpha(t)=\alpha_{0}+\mu_{\alpha} t+\pi f_{\alpha}(t)+a_{1} Q_{\alpha}(t), \\
\delta(t)=\delta_{0}+\mu_{\delta} t+\pi f_{\delta}(t)+a_{1} Q_{\delta}(t),
\end{gathered}
$$

where $Q_{\alpha}$ and $Q_{\delta}$ are the orbital factors. They are given by (see, e.g., van de Kamp 1967)

$$
Q_{\alpha}=B^{\prime} x(t)+G^{\prime} y(t)
$$


Table 3

VLBA Data in Taurus from the NRAO Archive

\begin{tabular}{|c|c|c|c|}
\hline Project Code & Date & Source & Calibrators $^{\mathrm{a}}$ \\
\hline BM198A & 2004 Mar 11 & V773 Tau A & $\mathrm{J} 0403+2600, \mathrm{~J} 0408+3032$ \\
\hline BM198B & 2004 Mar 12 & V773 Tau A & $\mathrm{J} 0403+2600, \mathrm{~J} 0408+3032$ \\
\hline BM198C & 2004 Mar 13 & V773 Tau A & $\mathrm{J} 0403+2600, \mathrm{~J} 0408+3032$ \\
\hline BM198D & 2004 Mar 14 & V773 Tau A & $\mathrm{J} 0403+2600, \mathrm{~J} 0408+3032$ \\
\hline BM198E & 2004 Mar 15 & V773 Tau A & $\mathrm{J} 0403+2600, \mathrm{~J} 0408+3032$ \\
\hline BM198F & 2004 Mar 16 & V773 Tau A & $\mathrm{J} 0403+2600, \mathrm{~J} 0408+3032$ \\
\hline BM198G & 2004 Mar 17 & V773 Tau A & $\mathrm{J} 0403+2600, \mathrm{~J} 0408+3032$ \\
\hline BL128AA & 2005 Sep 08 & V773 Tau A & $\mathrm{J} 0408+3032, \mathrm{~J} 0403+2600, \mathrm{~J} 0429+2724, \mathrm{~J} 0356+2903$ \\
\hline BL128AB & 2005 Nov 15 & V773 Tau A & $\mathrm{J} 0408+3032, \mathrm{~J} 0403+2600, \mathrm{~J} 0429+2724, \mathrm{~J} 0356+2903$ \\
\hline BL128AC & 2006 Jan 21 & V773 Tau A & $\mathrm{J} 0408+3032, \mathrm{~J} 0403+2600, \mathrm{~J} 0429+2724, \mathrm{~J} 0356+2903$ \\
\hline BL128AD & 2006 Apr 01 & V773 Tau A & $\mathrm{J} 0408+3032, \mathrm{~J} 0403+2600, \mathrm{~J} 0429+2724, \mathrm{~J} 0356+2903$ \\
\hline BL128AE & 2006 Jun 12 & V773 Tau A & $\mathrm{J} 0408+3032, \mathrm{~J} 0403+2600, \mathrm{~J} 0429+2724, \mathrm{~J} 0356+2903$ \\
\hline BL128AF & 2006 Sep 05 & V773 Tau A & $\mathrm{J} 0408+3032, \mathrm{~J} 0403+2600, \mathrm{~J} 0429+2724, \mathrm{~J} 0356+2903$ \\
\hline BL146B & 2007 Aug 23 & V773 Tau A & $\mathrm{J} 0408+3032, \mathrm{~J} 0403+2600, \mathrm{~J} 0429+2724, \mathrm{~J} 0356+2903$ \\
\hline BL146C & 2007 Aug 29 & V773 Tau A & $\mathrm{J} 0408+3032, \mathrm{~J} 0403+2600, \mathrm{~J} 0429+2724, \mathrm{~J} 0356+2903$ \\
\hline BL146D & 2007 Sep 05 & V773 Tau A & $\mathrm{J} 0408+3032, \mathrm{~J} 0403+2600, \mathrm{~J} 0429+2724, \mathrm{~J} 0356+2903$ \\
\hline BL146E & 2007 Sep 11 & V773 Tau A & $\mathrm{J} 0408+3032, \mathrm{~J} 0403+2600, \mathrm{~J} 0429+2724, \mathrm{~J} 0356+2903$ \\
\hline BL146F & 2007 Sep 16 & V773 Tau A & $\mathrm{J} 0408+3032, \mathrm{~J} 0403+2600, \mathrm{~J} 0429+2724, \mathrm{~J} 0356+2903$ \\
\hline BL146G & 2007 Sep 21 & V773 Tau A & $\mathrm{J} 0408+3032, \mathrm{~J} 0403+2600, \mathrm{~J} 0429+2724, \mathrm{~J} 0356+2903$ \\
\hline BL146H & 2007 Sep 27 & V773 Tau A & $\mathrm{J} 0408+3032, \mathrm{~J} 0403+2600, \mathrm{~J} 0429+2724, \mathrm{~J} 0356+2903$ \\
\hline BL146I & 2007 Oct 03 & V773 Tau A & $\mathrm{J} 0408+3032, \mathrm{~J} 0403+2600, \mathrm{~J} 0429+2724, \mathrm{~J} 0356+2903$ \\
\hline BL146J & 2007 Oct 09 & V773 Tau A & $\mathrm{J} 0408+3032, \mathrm{~J} 0403+2600, \mathrm{~J} 0429+2724, \mathrm{~J} 0356+2903$ \\
\hline BL146K & 2007 Oct 17 & V773 Tau A & $\mathrm{J} 0408+3032, \mathrm{~J} 0403+2600, \mathrm{~J} 0429+2724, \mathrm{~J} 0356+2903$ \\
\hline BL146L & 2007 Oct 23 & V773 Tau A & $\mathrm{J} 0408+3032, \mathrm{~J} 0403+2600, \mathrm{~J} 0429+2724, \mathrm{~J} 0356+2903$ \\
\hline BL146M & 2007 Oct 27 & V773 Tau A & $\mathrm{J} 0408+3032, \mathrm{~J} 0403+2600, \mathrm{~J} 0429+2724, \mathrm{~J} 0356+2903$ \\
\hline BL146N & 2007 Nov 17 & V773 Tau A & $\mathrm{J} 0408+3032, \mathrm{~J} 0403+2600, \mathrm{~J} 0429+2724, \mathrm{~J} 0356+2903$ \\
\hline BM306 & 2009 Sep 27 & V773 Tau A & $\mathrm{J} 0408+3032, \mathrm{~J} 0403+2600, \mathrm{~J} 0429+2724, \mathrm{~J} 0356+2903$ \\
\hline BL124BA & 2004 Sep 19 & V1023 Tau & $\mathrm{J} 0429+2724, \mathrm{~J} 0433+2905, \mathrm{~J} 0408+3032, \mathrm{~J} 0403+2600$ \\
\hline BL124BB & 2005 Jan 04 & V1023 Tau & $\mathrm{J} 0429+2724, \mathrm{~J} 0433+2905, \mathrm{~J} 0408+3032, \mathrm{~J} 0403+260 \mathrm{C}$ \\
\hline BL124BC & 2005 Mar 25 & V1023 Tau & $\mathrm{J} 0429+2724, \mathrm{~J} 0433+2905, \mathrm{~J} 0408+3032, \mathrm{~J} 0403+2600$ \\
\hline BL124BD & 2005 Jul 04 & V1023 Tau & $\mathrm{J} 0429+2724, \mathrm{~J} 0433+2905, \mathrm{~J} 0408+3032, \mathrm{~J} 0403+260 \mathrm{C}$ \\
\hline BL136AA & 2005 Sep 18 & V1023 Tau & $\mathrm{J} 0429+2724, \mathrm{~J} 0433+2905, \mathrm{~J} 0408+3032, \mathrm{~J} 0403+2600$ \\
\hline BL136AB & 2005 Dec 28 & V1023 Tau & $\mathrm{J} 0429+2724, \mathrm{~J} 0433+2905, \mathrm{~J} 0408+3032, \mathrm{~J} 0403+260 \mathrm{C}$ \\
\hline BL124CA & 2004 Sep 22 & HDE 283572 & $\mathrm{~J} 0429+2724, \mathrm{~J} 0433+2905, \mathrm{~J} 0408+3032, \mathrm{~J} 0403+260 \mathrm{C}$ \\
\hline BL124CB & 2005 Jan 06 & HDE 283572 & $\mathrm{~J} 0429+2724, \mathrm{~J} 0433+2905, \mathrm{~J} 0408+3032, \mathrm{~J} 0403+260 \mathrm{C}$ \\
\hline BL124CC & 2005 Mar 30 & HDE 283572 & $\mathrm{~J} 0429+2724, \mathrm{~J} 0433+2905, \mathrm{~J} 0408+3032, \mathrm{~J} 0403+260 \mathrm{C}$ \\
\hline BL124CD & 2005 Jun 23 & HDE 283572 & $\mathrm{~J} 0429+2724, \mathrm{~J} 0433+2905, \mathrm{~J} 0408+3032, \mathrm{~J} 0403+260 \mathrm{C}$ \\
\hline BL136BA & 2005 Sep 23 & HDE 283572 & $\mathrm{~J} 0429+2724, \mathrm{~J} 0433+2905, \mathrm{~J} 0408+3032, \mathrm{~J} 0403+2600$ \\
\hline BL136BB & 2005 Dec 24 & HDE 283572 & $\mathrm{~J} 0429+2724, \mathrm{~J} 0433+2905, \mathrm{~J} 0408+3032, \mathrm{~J} 0403+2600$ \\
\hline BL128BA & 2005 Sep 07 & HP Tau G2 & $\mathrm{J} 0426+2327, \mathrm{~J} 0435+2532, \mathrm{~J} 0449+1754$ \\
\hline BL128BB & 2005 Nov 16 & HP Tau G2 & $\mathrm{J} 0426+2327, \mathrm{~J} 0435+2532, \mathrm{~J} 0449+1754$ \\
\hline BL128BC & 2006 Jan 23 & HP Tau G2 & $\mathrm{J} 0426+2327, \mathrm{~J} 0435+2532, \mathrm{~J} 0449+1754$ \\
\hline BL128BD & 2006 Mar 31 & HP Tau G2 & $\mathrm{J} 0426+2327, \mathrm{~J} 0435+2532, \mathrm{~J} 0449+1754$ \\
\hline BL128BE & 2006 Jun 10 & HP Tau G2 & $\mathrm{J} 0426+2327, \mathrm{~J} 0435+2532, \mathrm{~J} 0449+1754$ \\
\hline BL128BF & 2006 Sep 08 & HP Tau G2 & $\mathrm{J} 0426+2327, \mathrm{~J} 0435+2532, \mathrm{~J} 0449+1754$ \\
\hline ВТ093АВ & 2007 Sep 03 & HP Tau G2 & $\mathrm{J} 0426+2327, \mathrm{~J} 0435+2532, \mathrm{~J} 0449+1754$ \\
\hline BT093AC & 2007 Dec 04 & HP Tau G2 & $\mathrm{J} 0426+2327, \mathrm{~J} 0435+2532, \mathrm{~J} 0449+1754$ \\
\hline BL118A & 2003 Sep 24 & $\mathrm{~T}$ Tau Sb & $\mathrm{J} 0428+1732, \mathrm{~J} 0431+1731$ \\
\hline BL118B & 2003 Nov 18 & $\mathrm{~T}$ Tau Sb & $\mathrm{J} 0428+1732, \mathrm{~J} 0431+1731$ \\
\hline BL118C & 2004 Jan 15 & $\mathrm{~T}$ Tau Sb & $\mathrm{J} 0428+1732, \mathrm{~J} 0431+1731$ \\
\hline BL118D & 2004 Mar 26 & $\mathrm{~T}$ Tau Sb & $\mathrm{J} 0428+1732, \mathrm{~J} 0431+1731$ \\
\hline BL118E & 2004 May 13 & $\mathrm{~T}$ Tau Sb & $\mathrm{J} 0428+1732, \mathrm{~J} 0431+1731$ \\
\hline BL118F & 2004 Jul 08 & $\mathrm{~T}$ Tau Sb & $\mathrm{J} 0428+1732, \mathrm{~J} 0431+1731$ \\
\hline BL124AA & 2004 Sep 16 & $\mathrm{~T}$ Tau Sb & $\mathrm{J} 0428+1732, \mathrm{~J} 0426+2327, \mathrm{~J} 0412+1856$ \\
\hline BL124AB & 2004 Nov 09 & $\mathrm{~T}$ Tau Sb & $\mathrm{J} 0428+1732, \mathrm{~J} 0426+2327, \mathrm{~J} 0412+1856$ \\
\hline BL124AC & 2004 Dec 28 & $\mathrm{~T}$ Tau Sb & $\mathrm{J} 0428+1732, \mathrm{~J} 0426+2327, \mathrm{~J} 0412+1856$ \\
\hline BL124AD & 2005 Feb 23 & $\mathrm{~T}$ Tau Sb & $\mathrm{J} 0428+1732, \mathrm{~J} 0426+2327, \mathrm{~J} 0412+1856$ \\
\hline BL124AE & 2005 May 09 & $\mathrm{~T}$ Tau Sb & $\mathrm{J} 0428+1732, \mathrm{~J} 0426+2327, \mathrm{~J} 0412+1856$ \\
\hline BL124AF & 2005 Jul 08 & $\mathrm{~T}$ Tau Sb & $\mathrm{J} 0428+1732, \mathrm{~J} 0426+2327, \mathrm{~J} 0412+1856$ \\
\hline BS171 & 2007 Mar 10 & V807 Tau B & $\mathrm{J} 0426+2327, \mathrm{~J} 0429+2724, \mathrm{~J} 0450+2249$ \\
\hline BS176A & 2008 Mar 19 & V807 Tau B & $\mathrm{J} 0426+2327, \mathrm{~J} 0429+2724, \mathrm{~J} 0450+2249$ \\
\hline
\end{tabular}


Table 3

(Continued)

\begin{tabular}{lccc}
\hline \hline Project Code & Date & Source & Calibrators $^{\mathrm{a}}$ \\
\hline BS176B & 2008 Jun 04 & V807 Tau B & J0426+2327, J0429+2724, J0450+2249 \\
BS176C & 2008 Aug 28 & V807 Tau B & J0426+2327, J0429+2724, J0450+2249 \\
BS176D & 2008 Dec 10 & V807 Tau B & J0426+2327, J0429+2724, J0450+2249 \\
BS176E & 2009 Mar 06 & V807 Tau B & J0426+2327, J0429+2724, J0450+2249 \\
\hline
\end{tabular}

Note.

${ }^{\mathrm{a}}$ The main calibrator is the first source in the list.

Table 4

VLBA Measured Positions for the Sources in Our Sample

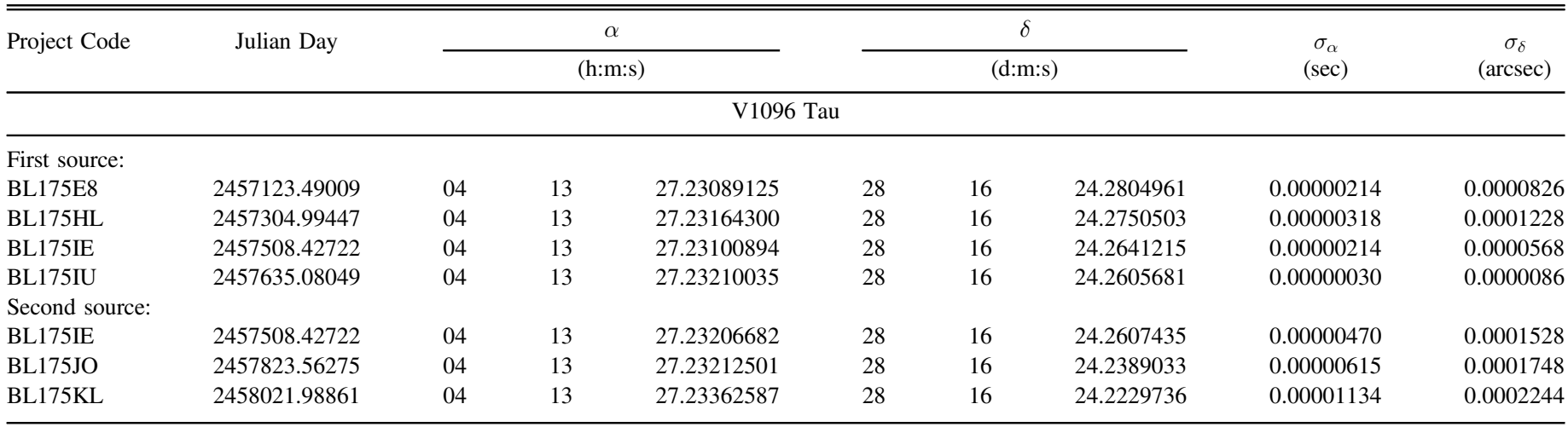

(This table is available in its entirety in machine-readable form.)

$$
Q_{\delta}=A^{\prime} x(t)+F^{\prime} y(t),
$$

where the orientation factors $B^{\prime}, A^{\prime}, G^{\prime}, F^{\prime}$ are related to the Thiele-Innes constants as follows:

$$
\begin{aligned}
& B^{\prime}=-\cos \omega \sin \Omega-\sin \omega \cos \Omega \cos i, \\
& A^{\prime}=-\cos \omega \cos \Omega+\sin \omega \sin \Omega \cos i, \\
& G^{\prime}=+\sin \omega \sin \Omega-\cos \omega \cos \Omega \cos i, \\
& F^{\prime}=+\sin \omega \cos \Omega+\cos \omega \sin \Omega \cos i .
\end{aligned}
$$

The elliptical rectangular coordinates $x(t)$ and $y(t)$ are given by

$$
\begin{gathered}
x(t)=\cos E(t)-e, \\
y(t)=\sin E(t) \sqrt{1-e^{2}},
\end{gathered}
$$

where $E(t)$ is the eccentric anomaly given by Kepler's transcendental equation. Similar equations hold for the secondary component. In this case, we replace $a_{1}$ by the semimajor axis $a_{2}$ of the secondary, which is scaled by the mass ratio $q$ of the two components.

To simplify the forthcoming discussion, we denote the methodology described above to solve simultaneously for the astrometric parameters and orbital elements of the binary system using absolute positions by the name "full model." Some binaries in our sample also exhibit relative astrometry of the two components from optical and near-infrared (NIR) observations published in previous works that have been incorporated into our study. In this case, our analysis is limited to the right-hand term of Equations (7) and (8) to derive the orbital elements of the binary system. The resulting semimajor axis $a$ refers to the relative orbit of the two components. We denote this solution using relative positions by the name "relative model." Finally, we combine the absolute positions measured in this work and relative positions from the literature to perform a "joint fit" solution.

\subsection{Solving the System of Equations}

The source positions obtained from the JMFIT task in AIPS were used to derive the astrometric and orbital parameters of the stars in our sample. Table 4 lists the measured positions for each source in our sample. However, it is important to mention that the positional uncertainties provided by JMFIT, which roughly represent the expected astrometric precision delivered by the VLBA, do not include various systematic errors that may significantly affect the accuracy of the computed astrometric parameters (see, e.g., Pradel et al. 2006). To overcome this problem, additional errors were added quadratically to the positional errors given by JMFIT to adjust the reduced $\chi^{2}$ value in the astrometric fit to unity (see also Menten et al. 2007; Ortiz-León et al. 2017b). These additional errors range in most cases from 0.05 to 0.30 mas for both single stars and binaries (the few exceptions are discussed in Section 4).

To solve for the astrometric and orbital elements of the star (or stellar system), we developed our own routine in the Python programming language based on the emcee package (ForemanMackey et al. 2013), which implements the Markov chain Monte Carlo (MCMC) method proposed by Goodman \& Weare (2010). The algorithm was adapted to our purposes and applied to the general problem of computing the astrometric and orbital parameters from the astrometry fitting. Briefly, the method that we use in this work explores the parameter space using a number of walkers and iteration steps to solve the system of equations presented in Sections 3.1 and 3.2 via Bayesian inference. The walkers move around the $n$-dimensional parameter space and take tentative steps toward the lowest value of $\chi^{2}$. 


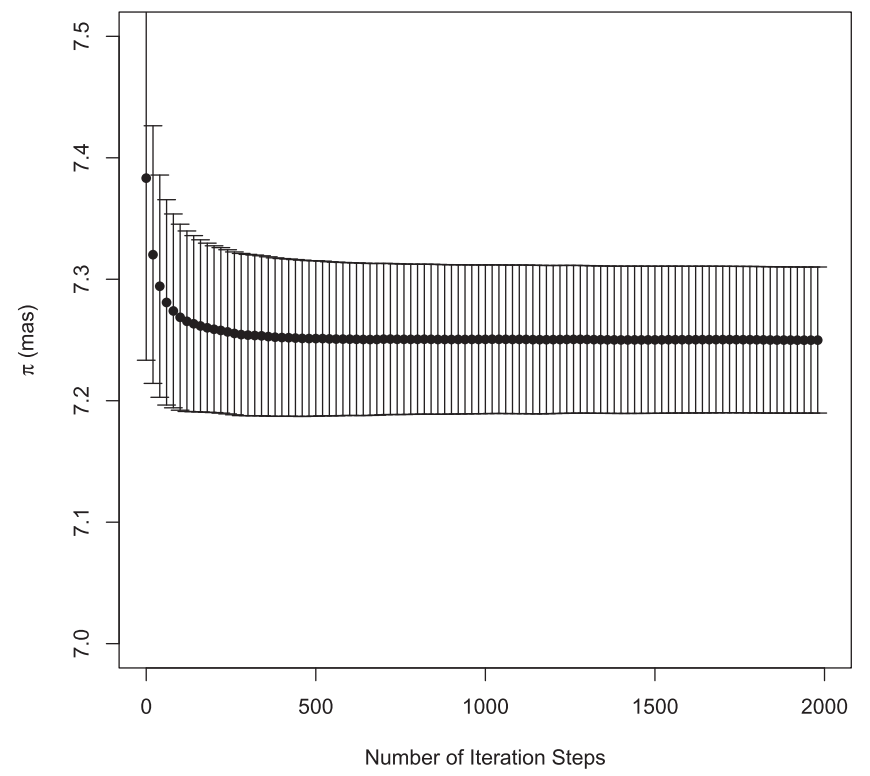

Figure 3. Convergence of the trigonometric parallax obtained for LFAM 8 based on the MCMC method implemented in this work. Each point is the average of the trigonometric parallax obtained by 200 walkers for each iteration step, and the error bars indicate the scatter of the solution given by the ensemble of walkers.

This produces a distribution of the individual solutions given by the ensemble of walkers. We run the MCMC method typically with 200 walkers to sample the distribution of each parameter with a significant number of individual solutions. Then, we take the mean and standard deviation of the distribution of each parameter as our final result.

Our methodology based on the MCMC method was first applied to the sources previously investigated by our team in the Ophiuchus region (Ortiz-León et al. 2017b) for calibration purposes. We varied the number of iterations for each walker from 1 to 2000 steps and verified that convergence of the Markov chains of the ensemble of walkers is attained after $\sim 100$ iterations steps where the mean (and median) of the computed parameters are clearly bounded by the variance of the sample. Figure 3 illustrates the convergence of our results for the Ophiuchus source LFAM 8 as an example. We find a trigonometric parallax of $\pi=7.242 \pm 0.060$ mas based on the MCMC methodology described in this section, which is in good agreement with the result of $\pi=7.246 \pm 0.088$ mas reported by Ortiz-León et al. (2017b). Thus, the solutions based on the MCMC method presented in this paper are calculated using 500 iteration steps as a conservative threshold to ensure convergence of the Markov chains for both single stars and binaries in our sample.

\section{Results}

We present trigonometric parallaxes for 18 stars in our sample with a minimum of three detections during our observing campaign. The trigonometric parallaxes and proper motions derived in this study are listed in Table 5. In addition, we derived the orbital elements of six binary and multiple systems in our sample, which are given in Table 6. The best-fit solutions of our results are collectively illustrated in Figures 4 and 5. In Table 7 we compare the astrometry derived from the full model and joint fit for the binaries with measured relative positions in the literature. The relative astrometry model for these sources is illustrated in Figure 6. In the following we comment on the properties of the individual stars (and stellar systems) that have been investigated. We present our discussion about individual sources in the order that they appear in Table 2.

\subsection{V1096 Tau}

V1096 Tau is a binary system that could be resolved in our observations. Both components were simultaneously detected in only one epoch (project BL175IE), where the primary component of the system V1096 Tau A exhibits a flux density of $0.71 \pm 0.05 \mathrm{mJy}$ that is almost twice that of the secondary component (V1096 Tau B), with $0.36 \pm 0.05 \mathrm{mJy}$. We detected the primary and secondary, respectively, in a total of four and three epochs. However, our observations cover a small fraction of the orbit so that the fit including the orbital motion of the system does not converge. Hence, we fit the measured positions of the individual components solely for the proper motion and parallax. We included the acceleration term in the astrometry fit for the primary component, which indeed represents a better description to the data, but this was not possible for the secondary due to the limited number of detections available. Our result for the secondary component is only indicative and should be regarded with caution, as it is likely to be biased by the noncorrected orbital motion of the system. The systematic errors that we add to the stellar positions of V1096 Tau A and V1096 Tau B (as described in Section 3.3) reach up to 1 and 3 mas, respectively, and they are significantly larger than the other stars. The weighted mean parallax of the results given in Table 5 for the two components is $\pi=8.04 \pm 0.50$ mas. This is consistent with a distance estimate of $d=124.4_{-7.2}^{+8.2} \mathrm{pc}$. Despite the admittedly large errors on our results, this is the first distance determination for this binary system to date. The weighted mean proper motions of the two components are $\mu_{\alpha} \cos \delta=2.612 \pm 0.691 \mathrm{mas} \mathrm{yr}^{-1}$ and $\mu_{\delta}=-17.372 \pm 0.676$ mas yr $^{-1}$. V1096 Tau continues to be monitored by our team, and the results presented in this paper will be refined when more observations become available.

\subsection{V773 Tau}

V773 Tau is a well-known quadruple system (see, e.g., Duchêne et al. 2003; Woitas 2003), and the source detected in our observations is the primary component V773 Tau A, which itself is a tight binary. V773 Tau A has been detected in a total of seven epochs during our observing, but a simultaneous detection of both components occurred only in three epochs (see Table 4). Moreover, it has also been observed with the VLBA in 27 additional epochs between 2004 March and 2009 September for projects BM198, BL128, BL146, and BM306 (see Table 3). We note that project BM198 observed $\mathrm{J0403+2600}$ as the main phase calibrator while the other projects (including GOBELINS) used J0408+3032 instead. We corrected the positions measured in BM198 before combining them with the other projects. The mean position of $\mathrm{J} 0403+2600$ measured in projects BM198 is $\alpha=04^{\mathrm{h}} 03^{\mathrm{m}} 05.586049$ and $\delta=26^{\circ} 00^{\prime} 01$ " 50275 . The mean position of $\mathrm{J} 0403+2600$ (relative to $\mathrm{J} 0408+3032$ ) in the other projects is $\alpha=04^{\mathrm{h}} 03^{\mathrm{m}} 05^{\mathrm{s}} .586052$ and $\delta=26^{\circ} 00^{\prime} 01$ ". 50137 . Hence, we applied a correction of $\Delta \alpha=0.000003 \mathrm{~s}$ and $\Delta \delta=-0$ ". 00138 to the measured positions in project BM198. Another important point to mention about projects BM198 
Table 5

Proper Motions, Parallaxes, and Distances Derived from VLBA Observations

\begin{tabular}{|c|c|c|c|c|c|c|}
\hline Identifier & $\begin{array}{c}\mu_{\alpha} \cos \delta \\
\left(\mathrm{mas} \mathrm{yr}^{-1}\right)\end{array}$ & $\begin{array}{c}\mu_{\delta} \\
\left(\operatorname{mas~yr}^{-1}\right)\end{array}$ & $\begin{array}{c}a_{\alpha} \cos \delta \\
\left(\mathrm{mas}_{\mathrm{yr}}^{-2}\right)\end{array}$ & $\begin{array}{c}a_{\delta} \\
\left(\operatorname{mas~yr}^{-2}\right)\end{array}$ & $\begin{array}{c}\text { Parallax } \\
\text { (mas) }\end{array}$ & $\begin{array}{c}\text { Distance } \\
(\mathrm{pc})\end{array}$ \\
\hline V1096 Tau A & $2.089 \pm 0.730$ & $-16.167 \pm 0.711$ & $11.640 \pm 2.945$ & $0.234 \pm 3.710$ & $8.055 \pm 0.535$ & $124.1_{-7.7}^{+8.8}$ \\
\hline V1096 Tau B & $7.147 \pm 2.149$ & $-28.765 \pm 2.186$ & $\ldots$ & $\ldots$ & $7.924 \pm 1.334$ & $126.2_{-18.2}^{+25.5}$ \\
\hline V773 Tau A & $10.253 \pm 0.8434$ & $-25.119 \pm 0.301$ & $\ldots$ & $\ldots$ & $7.692 \pm 0.085$ & $130.0_{-1.4}^{+1.5}$ \\
\hline V1098 Tau & $11.148 \pm 0.175$ & $-27.327 \pm 0.172$ & $\cdots$ & $\cdots$ & $8.070 \pm 0.310$ & $123.9_{-4.6}^{+5.0}$ \\
\hline 2MASS J04182909+2826191 & $8.384 \pm 0.195$ & $-19.627 \pm 0.217$ & $\ldots$ & $\ldots$ & $7.583 \pm 0.389$ & $131.9_{-6.4}^{+7.1}$ \\
\hline HD 283518 & $8.703 \pm 0.017$ & $-24.985 \pm 0.020$ & $\cdots$ & $\cdots$ & $7.751 \pm 0.027$ & $129.0_{-0.5}^{+0.5}$ \\
\hline V1023 Tau & $8.371 \pm 0.020$ & $-25.490 \pm 0.020$ & $\cdots$ & $\cdots$ & $7.686 \pm 0.032$ & $130.1_{-0.5}^{+0.5}$ \\
\hline $\mathrm{T}$ Tau Sb & $6.790 \pm 0.432$ & $-11.131 \pm 0.444$ & $\cdots$ & $\cdots$ & $6.723 \pm 0.046$ & $148.7_{-1.0}^{+1.0}$ \\
\hline V1201 Tau B & $10.839 \pm 0.050$ & $-13.235 \pm 0.058$ & $0.335 \pm 0.076$ & $0.147 \pm 0.071$ & $6.363 \pm 0.069$ & $157.2_{-1.7}^{+1.7}$ \\
\hline HD 283641 & $10.913 \pm 0.037$ & $-16.772 \pm 0.044$ & $\ldots$ & $\ldots$ & $6.285 \pm 0.070$ & $159.1_{-1.8}^{+1.8}$ \\
\hline XZ Tau A & $10.858 \pm 0.027$ & $-16.264 \pm 0.060$ & $\cdots$ & $\cdots$ & $6.793 \pm 0.025$ & $147.2_{-0.5}^{+0.5}$ \\
\hline V807 Tau B & $8.573 \pm 0.068$ & $-28.774 \pm 0.201$ & $\ldots$ & $\ldots$ & $7.899 \pm 0.105$ & $126.6_{-1.7}^{+1.7}$ \\
\hline V1110 Tau & $-52.705 \pm 0.062$ & $-11.321 \pm 0.066$ & $\cdots$ & $\cdots$ & $11.881 \pm 0.149$ & $84.2_{-1.0}^{+1.1}$ \\
\hline HP Tau G2 & $11.248 \pm 0.022$ & $-15.686 \pm 0.013$ & $\cdots$ & $\cdots$ & $6.145 \pm 0.029$ & $162.7_{-0.8}^{+0.8}$ \\
\hline V999 Tau & $9.533 \pm 0.218$ & $-15.684 \pm 0.198$ & $\cdots$ & $\cdots$ & $6.972 \pm 0.197$ & $143.4_{-3.9}^{+4.2}$ \\
\hline V1000 Tau & $6.010 \pm 0.235$ & $-17.720 \pm 0.159$ & $\cdots$ & $\cdots$ & $7.324 \pm 0.132$ & $136.5_{-2.4}^{+2.5}$ \\
\hline HD 282630 & $3.897 \pm 0.113$ & $-24.210 \pm 0.132$ & $\ldots$ & $\ldots$ & $7.061 \pm 0.125$ & $141.6_{-2.5}^{+2.6}$ \\
\hline HDE 283572 & $8.853 \pm 0.096$ & $-26.491 \pm 0.113$ & $\cdots$ & $\cdots$ & $7.722 \pm 0.057$ & $129.5_{-0.9}^{+1.0}$ \\
\hline
\end{tabular}

is that these observations include data collected with both the VLBA and the Effelsberg antenna. But for consistency with the rest of the data used in this work, we decided to remove the Effelsberg antenna from the data reduction. The same applies to the BM306 project, which was observed with the High Sensitivity Array (HSA). We removed the external antennae and calibrated the observation using only VLBA data.

The orbital parameters of the short-period binary system V773 Tau Aa-Ab have already been constrained by Torres et al. (2012) based on the past VLBA observations listed in Table 3, supplemented by radial velocity observations from Boden et al. (2007). They derived the dynamical masses of the two components $\left(m_{\mathrm{Aa}}=1.55 \pm 0.11 M_{\odot}\right.$ and $m_{\mathrm{Ab}}=$ $\left.1.293 \pm 0.068 M_{\odot}\right)$ and a trigonometric parallax of $\pi=$ $7.70 \pm 0.19$ mas computed from the positions of the barycenter of the system, including a uniformly accelerated proper motion in the analysis. As explained in their study, the model including a uniform acceleration (see Section 3.2) provided a good description to the data, because their observations covered a small fraction of the orbit of the V773 Tau A-B system. However, this not the case when we include the more recent data from the GOBELINS project in this analysis, which increases the time base of collected observations to about $13 \mathrm{yr}$. We computed the barycentric positions of the V773 Tau Aa-Ab system for the three epochs with simultaneous detections of the two components in our observing campaign and combined them with the recalibrated barycentric positions reported by Torres et al. (2012). We verified that the model assuming a uniform acceleration indeed provides a poor fit to the data with the extended time base of our observations. We thus performed a full fit to the data, including the orbital motion of the V773 Tau A-B in the analysis. Doing so, we derive an orbital period of $P=20.3 \pm 0.8 \mathrm{yr}$ that is somewhat shorter than the value of $P=26.2 \pm 1.1 \mathrm{yr}$ obtained by Boden et al. (2012) based on relative astrometry and radial velocities. The resulting trigonometric parallax of $\pi=7.692 \pm$ 0.085 mas yields a distance estimate of $d=130.0_{-1.4}^{+1.5} \mathrm{pc}$ for the barycenter of the V773 Tau Aa-Ab system that is more accurate and precise than the result of $d=132.8 \pm 2.3 \mathrm{pc}$ obtained by Torres et al. (2012).

\subsection{V1098 Tau, 2MASS J04182909+2826191, V999 Tau, and HD 282630}

V1098 Tau, 2MASS J04182909+2826191, and HD 282630 have been detected in only three epochs of our observing campaign. On the other hand, V999 Tau has been detected in five different projects, but in practice this corresponds to only three epochs owing to the short time interval between projects BL175IC/BL175I7 and BL175JW/BL175JY. As a result, their trigonometric parallaxes are less precise as compared to the other stars in our sample with more detections. Because of the small number of detections, we computed the additional errors on the stellar positions (see Section 3.3) from the methodology outlined in Pradel et al. (2006). The resulting errors reach up to 0.8 mas depending on the position of the source in the sky and the angular separation to the main phase calibrator.

\section{4. $H D 283518$}

HD 283518 (V410 Tau) was systematically detected in every epoch of our observing campaign. It is known to be a multiple system (see, e.g., Harris et al. 2012), but only one component could be detected in our observations. We do not see the signature of the orbital motion in the stellar positions measured in this work, indicating that the gravitational effects of the secondary on the primary are negligible. Hence, we solved for the astrometric parameters of this source using the methodology described in Section 3.1 for single stars. We also compared our results with the alternative approach outlined in Section 3.2 using a uniform acceleration. Both methods return compatible results (with the same level of accuracy and precision), and the derived acceleration terms in the latter approach are consistent with zero. Thus, the results presented in Table 5 refer to the model without acceleration. The trigonometric parallax that we derive for HD 283518 has a 
Table 6

Orbital Elements of the Binaries and Multiple Systems in Our Sample

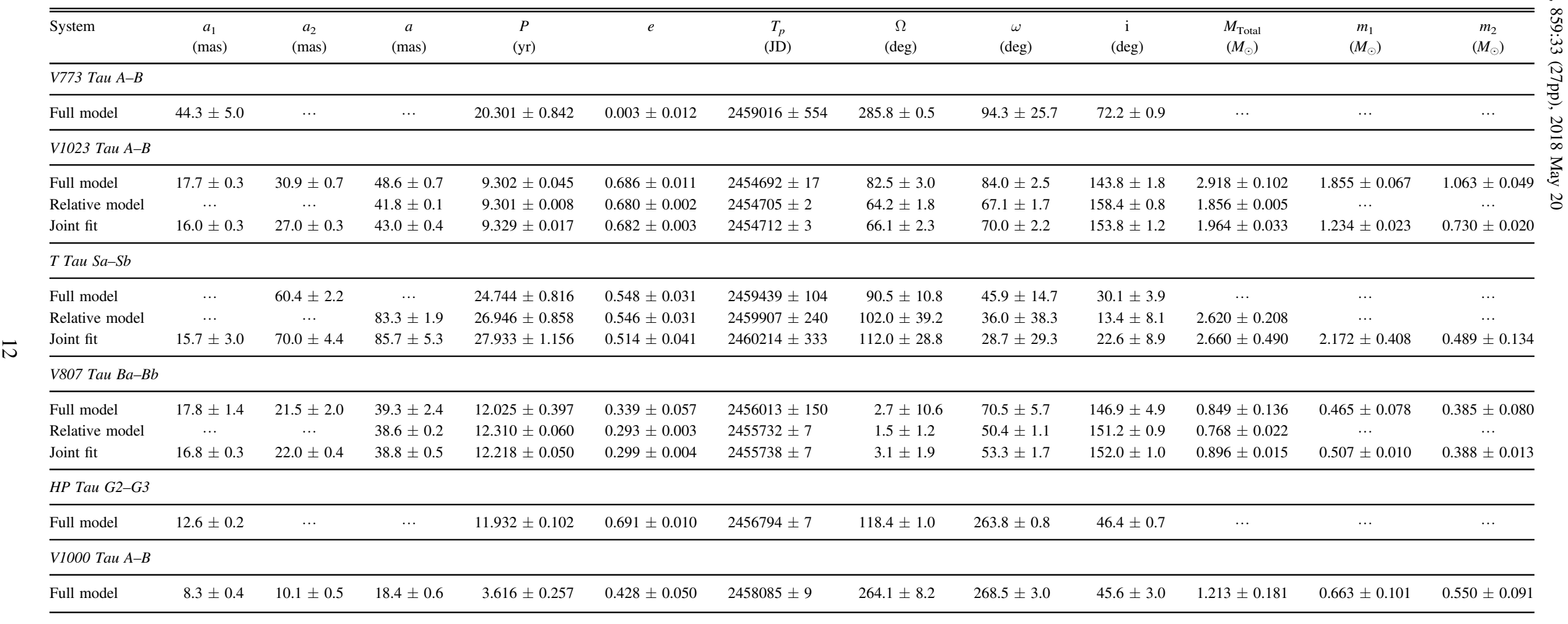



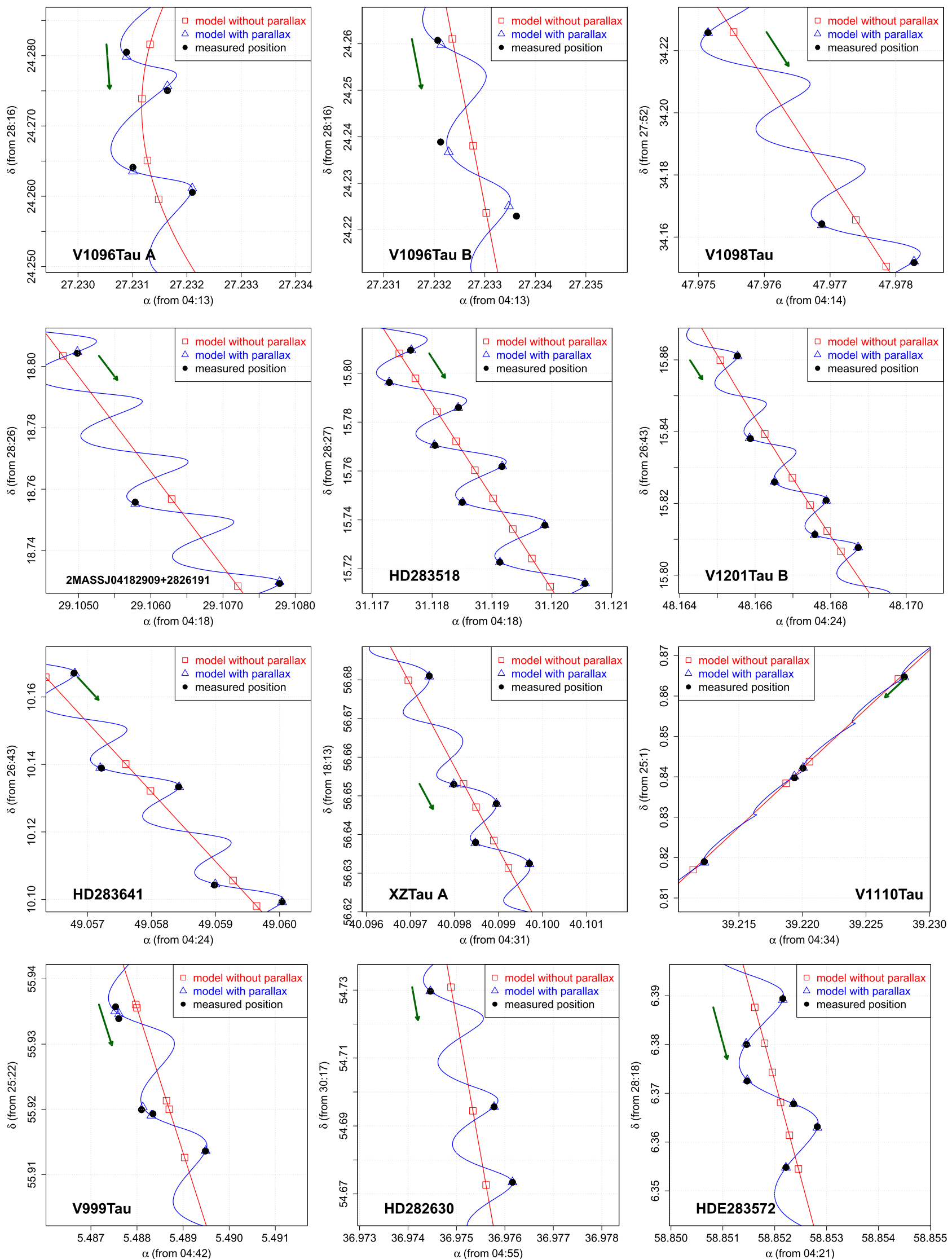

Figure 4. Astrometry fit for the single and long orbital period YSOs in our sample. Measured positions with the VLBA are shown as black circles and their corresponding error bars (including systematic errors) are mostly smaller than the symbols. The red line indicates the model with the parallax signature removed, and the blue line represents the model including the parallax. Red squares and blue triangles mark the expected positions for the corresponding models. The green arrow shows the direction of the stellar proper motions in the plane of the sky. 

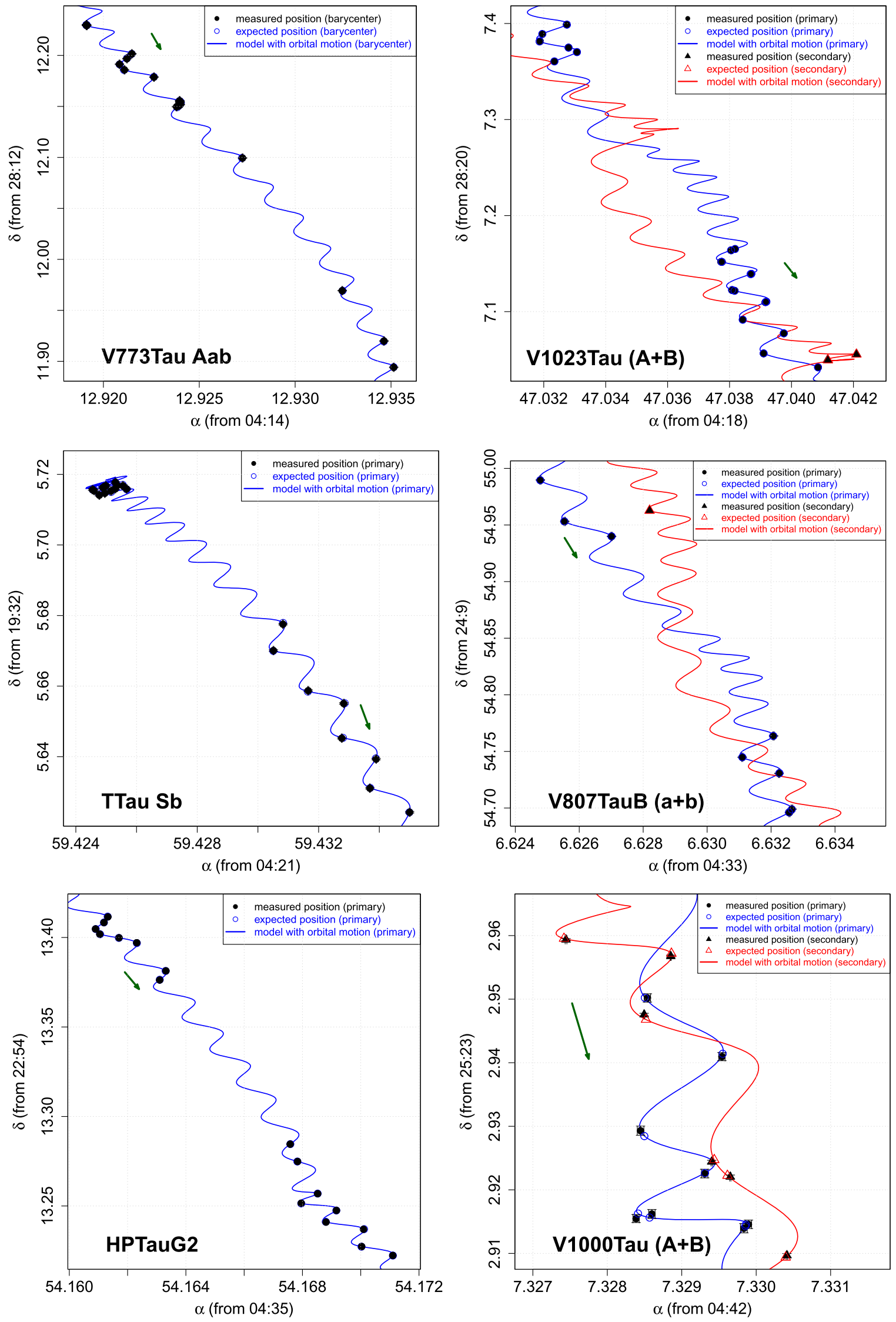

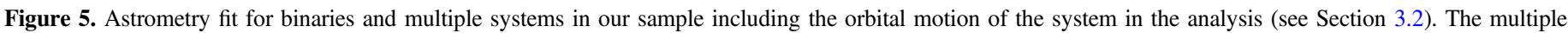

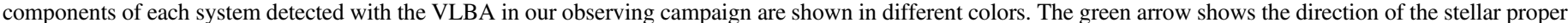
motions in the plane of the sky. 

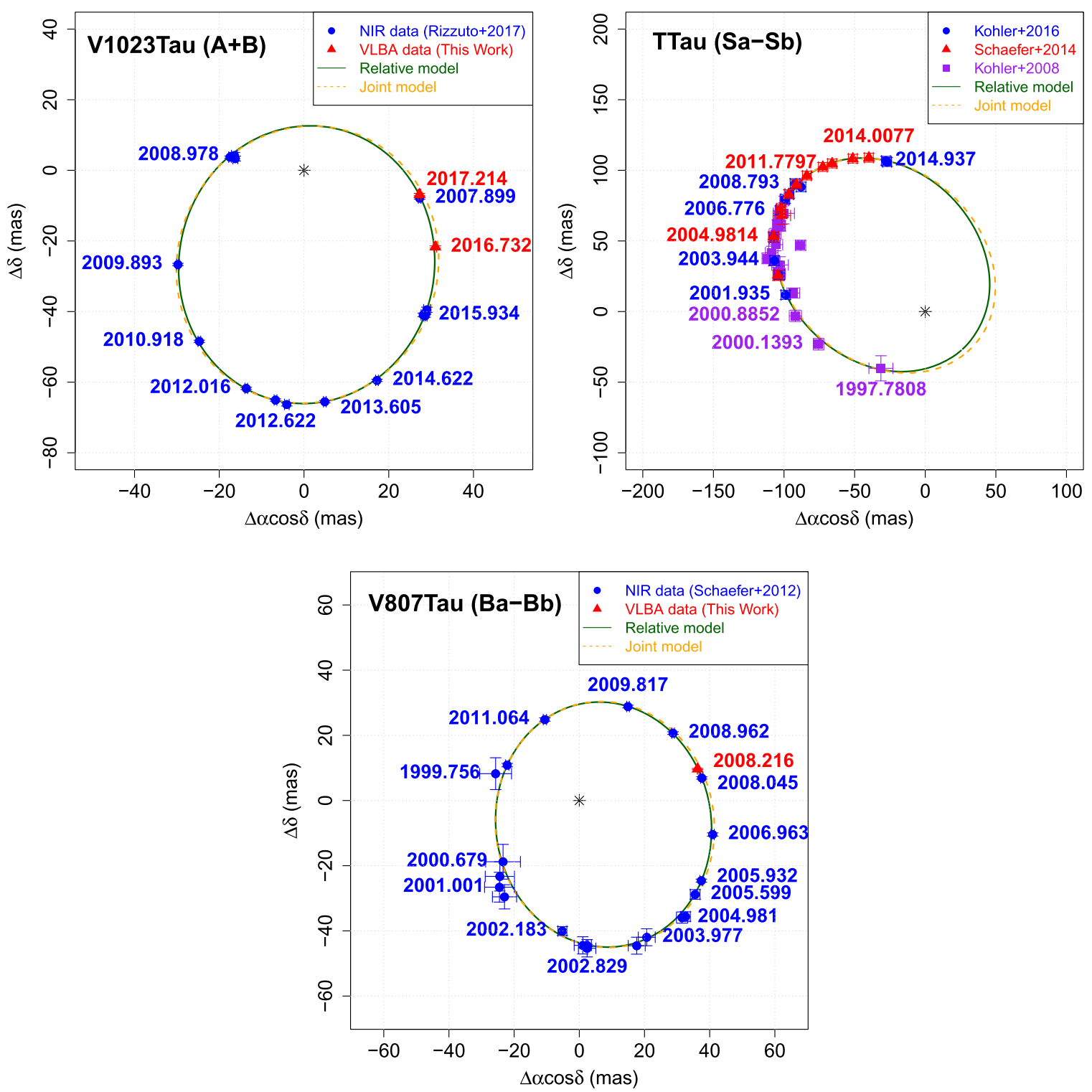

Figure 6. Relative astrometry of the components for three binary systems in our sample with measured relative positions in the literature. The black asterisk indicates the position of the secondary component (relative to the primary).

Table 7

Comparison of the Astrometry Derived for Binaries from Different Methods

\begin{tabular}{|c|c|c|c|c|}
\hline Method & $\begin{array}{c}\mu_{\alpha} \cos \delta \\
\left(\mathrm{mas} \mathrm{yr}^{-1}\right)\end{array}$ & $\begin{array}{c}\mu_{\delta} \\
\left(\operatorname{mas~yr}^{-1}\right)\end{array}$ & $\begin{array}{c}\text { Parallax } \\
\text { (mas) }\end{array}$ & $\begin{array}{l}\text { Distance } \\
(\mathrm{pc})\end{array}$ \\
\hline \multicolumn{5}{|c|}{ V1023 Tau } \\
\hline Joint fit & $8.393 \pm 0.019$ & $-25.489 \pm 0.026$ & $7.752 \pm 0.116$ & $129.0_{-1.9}^{+2.0}$ \\
\hline Weighted mean & $8.382 \pm 0.014$ & $-25.490 \pm 0.016$ & $7.691 \pm 0.031$ & $130.0_{-0.5}^{+0.5}$ \\
\hline Joint fit & $4.577 \pm 0.272$ & $-11.037 \pm 0.250$ & $6.723 \pm 0.046$ & $148.7_{-1.0}^{+1.0}$ \\
\hline Weighted mean & $4.619 \pm 0.205$ & $-10.564 \pm 0.162$ & $6.727 \pm 0.041$ & $148.7_{-0.9}^{+0.9}$ \\
\hline \multicolumn{5}{|c|}{ V807 Tаи В } \\
\hline Full model & $8.573 \pm 0.068$ & $-28.774 \pm 0.201$ & $7.899 \pm 0.105$ & $126.6_{-1.7}^{+1.7}$ \\
\hline
\end{tabular}


relative error of $0.3 \%$, making it the most precise result in our sample.

\subsection{V1023 Tau}

V1023 Tau (Hubble 4) is another binary system that was resolved in our observations. The primary component of this system is a weak-line T Tauri star of spectral type K7 (Nguyen et al. 2012), and it has been detected in a total of 14 epochs during our observing campaign. On the other hand, the secondary component could be detected in only two epochs. In addition, the primary has also been observed between 2004 September and 2005 December for projects BL124 and BL136. These data have already been analyzed and published by Torres et al. (2007). We combined these observations with the more recent GOBELINS data to fit the measured positions over a time base of $\sim 13 \mathrm{yr}$, which covers one full orbit of the system. Both data sets used the same main phase calibrator so that no correction has to be applied to the measured positions.

First, we performed a full fit of the orbit using only the VLBA observations reported in this paper and in Torres et al. (2007). Then, we combined the VLBA observations of projects BL175J2 and BL175JS where both components were simultaneously detected with the NIR relative astrometry from Rizzuto et al. (2018, submitted) to refine the orbital elements of the binary system. Finally, we combined our VLBA absolute positions with the NIR relative positions to perform a joint fit. Tables 6 and 7 compare our results from the different methods. The distance that we derive here from the full model $\left(d=130.1_{-0.5}^{+0.5} \mathrm{pc}\right)$ is somewhat shorter than the value of $d=132.8 \pm 0.5 \mathrm{pc}$ obtained by Torres et al. (2007) using VLBA data from projects BL124 and BL136. We argue that the latter result is likely biased by the noncorrected binarity of the source. By combining the VLBA absolute positions with the NIR relative astrometry, we find a distance of $d=129.0_{-1.9}^{+2.0} \mathrm{pc}$ that is less precise but fully consistent with our previous result. The weighted mean of both values yields $d=130.0_{-0.5}^{+0.5} \mathrm{pc}$, which is the most precise and accurate distance estimate for this source to date. Our analysis also made it possible to determine, for the first time, the dynamical masses of the individual components of this system $\left(m_{\mathrm{A}}=1.234 \pm 0.023 M_{\odot}\right.$ and $\left.m_{\mathrm{B}}=0.730 \pm 0.020 M_{\odot}\right)$. This implies a somewhat smaller mass ratio $(q=0.592 \pm 0.012)$ than the value of $q=0.73$ previously reported by Harris et al. (2012).

\subsection{T Tau}

T Tau is a well-known triple system (see, e.g., Duchêne et al. 2002), and the component detected in our observations with the VLBA is T Tau Sb. It has been detected in eight epochs during our observing campaign for the GOBELINS project. In addition, it was also observed by our team between 2003 September and 2005 July for projects BL118 and BL124 (see Table 3). However, we note that projects BL118 and BL128 observed J0428+1732 as the main phase calibrator, while GOBELINS uses it as a secondary calibrator (the main calibrator is J0412+1856). The mean position of J0428+1732 measured for projects BL118 and BL124 is $\alpha=04^{\mathrm{h}} 28^{\mathrm{m}} 35^{\mathrm{s}} .633679$ and $\delta=17^{\circ} 32^{\prime} 23$ " 58803 . In the GOBELINS campaign the mean position of $\mathrm{J} 0428+1732$ (relative to $\mathrm{J} 0412+1856$ ) is $\alpha=$ $04^{\mathrm{h}} 28^{\mathrm{m}} 35^{\mathrm{s}} .633685$ and $\delta=17^{\circ} 32^{\prime} 233^{\prime \prime} 58840$. Thus, we applied an offset of $\Delta \alpha=0.000006 \mathrm{~s}$ and $\Delta \delta=0.00037$ to the measured positions obtained from projects BL118 and BL124.
We fit the measured positions of T Tau Sb based on the full model to solve for the parallax and orbital motion of the binary system. The orbital elements obtained in this work (see Table 6) refer to the T Tau Sa-Sb system. We attempted to include an additional acceleration term due to the $\mathrm{T}$ Tau $\mathrm{N}$ component of the system, but the resulting acceleration parameter was consistent with zero. The results presented in Table 5 refer to our first solution (without acceleration). The distance that we derive in this paper for $\mathrm{T} \mathrm{Tau} \mathrm{Sb}$ $\left(d=148.3_{-2.1}^{+2.1} \mathrm{pc}\right)$ is in good agreement with the result of $d=147.6 \pm 0.6 \mathrm{pc}$ published previously by Loinard et al. (2007) using only data from projects BL118 and BL124. However, we consider our result to be more accurate because it takes into account the binarity/multiplicity of the source. Our solution obtained from the joint fit using the relative astrometry of the $\mathrm{T} \mathrm{Tau} \mathrm{Sa-Sb}$ published in previous studies yields a somewhat more precise distance estimate for this system $\left(d=148.7_{-1.0}^{+1.0} \mathrm{pc}\right)$ that confirms our first result. The weighted mean of both results yields a final distance of $d=148.7_{-0.9}^{+0.9} \mathrm{pc}$.

Other studies have already investigated the orbital motion of the $\mathrm{T}$ Tau $\mathrm{Sa}-\mathrm{Sb}$ system based on relative astrometry and different measurements (Köhler et al. 2008, 2016; Schaefer et al. 2014). As illustrated in Figure 6, we combined the relative positions from previous works to provide a more refined solution for the orbital parameters of the system. This analysis does not include VLBA data since our observations can only detect one component of the system. We note that most of the resulting orbital elements obtained in this paper including all measurements are more precise than the results reported in the individual studies. However, the errors on the individual parameters are still larger as compared to other stars in our sample. One reason for this result is the small coverage of the orbit, which requires further monitoring of the $\mathrm{T}$ Tau system.

\subsection{V1201 Tau and HD 283641}

V1201 Tau and HD 283641 are members of the same hierarchical multiple system, where both sources themselves are binary systems (see Kohler \& Leinert 1998; Mason et al. 2001). V1201 Tau and HD 283641 were also identified as wide binaries with a separation of 13!' 23 in a recent study conducted by Andrews et al. (2017) based on data from GaiaDR1. Indeed, their result is consistent with the mean angular separation of 14". 53 derived from our observations. The two components of the V1201 Tau system were simultaneously detected in only two epochs (projects BL175I1 and BL175KJ). We measured a flux density of $2.40 \pm 0.04 \mathrm{mJy}$ in project BL175I1 (2016 February 28) for the brightest component (hereafter V1201TauA), which significantly decreased to $0.15 \pm 0.05 \mathrm{mJy}$ in project BL175KJ (2017 September 18), becoming fainter than the so-defined secondary component V1201 Tau B. We derived a trigonometric parallax only for V1201 Tau B, which was detected in six epochs. We note from Figure 4 that the fit using a uniform acceleration represents a good description to the data, yielding a distance estimate of $d=157.2 \pm 1.7 \mathrm{pc}$. In the case of HD 283641 we detected only one component of the system in our observations. First, we fit the data as described in Section 3.1, and then we introduced the acceleration. We verified that the inclusion of a uniform acceleration in the model increases the errors on the astrometric parameters and that the derived acceleration terms are consistent with zero. As expected, the distance obtained for HD $283641(d=159.1 \pm 1.8 \mathrm{pc}$, without acceleration $)$ is 
compatible within $1 \sigma$ of the distance derived for V1201 Tau. Both sources are currently being monitored by our team, and we will deliver a more refined solution (including the orbital motion of the system) when these observations become available.

\subsection{XZ Tau}

The XZ Tau system is composed of two components (XZ Tau A and XZ Tau B) with angular separation of about 0"3 (Harris et al. 2012; Joncour et al. 2017). CarrascoGonzález et al. (2009) report on the detection of a third component (XZ Tau C) in this system separated by 0 ". 09 from XZ Tau A, making it a triple system. However, a more recent study conducted by Forgan et al. (2014) did not detect $\mathrm{XZ}$ Tau C and cast doubt on the existence of the third component. Only one component of this system could be detected in our observations, and we confirm that it corresponds to XZ Tau A. Krist et al. (2008) estimated that the minimum orbital period of the XZ Tau A-B system should be about $99 \mathrm{yr}$, which greatly exceeds the time base of our observations. Indeed, we see no evidence of binarity in our data, and modeling XZ Tau A as a single star (as described in Section 3.1) provides a good fit to the data (see Figure 4). Finally, it is interesting to note that all components of this system were suggested to be thermal radio sources (see Carrasco-González et al. 2009), but our study shows that XZ Tau A also produces nonthermal emission.

\subsection{V807 Tau}

V807 Tau is a hierarchical triple system, and the secondary component was resolved by Simon et al. (1995) into two close companions (V807 Tau Ba/Bb). The secondary has been detected in five epochs during the GOBELINS observing campaign. V807 Tau was also observed with the VLBA in the past (from 2007 March to 2009 March) by Schaefer et al. (2012) for projects BS171 and BS176. They report on three detections of V807 Tau Ba and one detection of V807 Tau Bb. Before combining these data with our own observations, we decided to download the files from the NRAO archive and reduce them by applying the same calibration procedure used in this work. Both data sets observed J0426+2327 as the main phase calibrator, so no correction needs to be applied to the positions measured in projects BS171 and BS176. We found that the source detected in the GOBELINS observations corresponds to V807 Tau Ba.

The model including a uniform acceleration produces a poor fit to the measured positions for V807 Tau Ba, because our observations cover almost one full orbit of the $\mathrm{Ba}-\mathrm{Bb}$ system. We thus performed a full fit, including the orbital motion of the close pair in our analysis, which indeed represents a better description of the data (see Figure 5). The distance that we derive from VLBA observations is $d=126.6 \pm 1.7 \mathrm{pc}$. Then, we used the NIR relative positions for the $\mathrm{Ba}-\mathrm{Bb}$ system obtained by Schaefer et al. (2012), together with the VLBA observation from project BS176A, where both components were simultaneously detected to refine the orbital parameters of the system from the relative model (see Figure 6). By combining the VLBA and NIR data, we find a distance of $d=131.8_{-2.3}^{+2.4} \mathrm{pc}$. The larger discrepancy in the distance estimates delivered by the full model and the joint fit (as compared to the other stars in Table 7) can be explained by the smaller number of data points (i.e., stellar positions) to fit the astrometry. The weighted mean parallax from both methods yields $d=128.5 \pm 1.4 \mathrm{pc}$, which is the first distance estimate for V807 Tau B to date.

The dynamical masses of the individual components $\left(m_{\mathrm{Ba}}=0.507 \pm 0.010 M_{\odot} \quad\right.$ and $\left.\quad m_{\mathrm{Bb}}=0.388 \pm 0.013 M_{\odot}\right)$ that we derive in this paper are more precise than the results obtained by Schaefer et al. (2012). As discussed in their study, they used the average distance of $140 \pm 10 \mathrm{pc}$ to the Taurus region to compute the stellar masses. We have rescaled the individual masses reported in their work to the distance that we derive in this paper, which gives $m_{\mathrm{Ba}}=0.471 \pm 0.018 M_{\odot}$ and $m_{\mathrm{Bb}}=0.397 \pm 0.017 M_{\odot}$. However, these numbers are still affected by the systematic error of $0.24 M_{\odot}$, which comes from the \pm 10 pc uncertainty in the distance used in their analysis. Both results are still in good agreement, but we argue that the dynamical masses derived in this paper are more accurate owing to the improved accuracy and precision of our distance determination.

\subsection{V1110 Tau}

V1110 Tau has been detected in four epochs during our observing campaign. The trigonometric parallax $(\pi=11.881 \pm$ $0.149 \mathrm{mas})$ and proper motion $\left(\mu_{\alpha} \cos \delta=-52.705 \pm\right.$ $\left.0.062 \mathrm{mas} \mathrm{yr}^{-1}, \mu_{\delta}=-11.321 \pm 0.066 \mathrm{mas} \mathrm{yr}^{-1}\right)$ that we derive here clearly confirm it as a foreground star not related to the Taurus star-forming clouds. This is also shown in Figure 2, where V1110 Tau clearly stands out with a position change rate

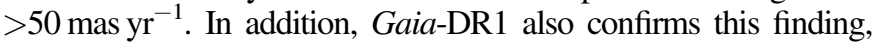
yielding a trigonometric parallax of $\pi=12.53 \pm 0.61$ mas and proper motion of $\mu_{\alpha} \cos \delta=-54.471 \pm 1.893 \mathrm{mas} \mathrm{yr}^{-1}, \mu_{\delta}=$ $-12.194 \pm 1.612{\text { mas } \mathrm{yr}^{-1}}^{-1}$. These values are consistent with but less precise than our results.

Interestingly, Martin et al. (1994) observed V1110 Tau (Wa Tau 1) and did not detect the Li I line in any of the two components of this binary system. Wahhaj et al. (2010) classified V1110 Tau as a weak-line T Tauri star of spectral type $\mathrm{K} 0$ and effective temperature of $5250 \mathrm{~K}$. They used the distance of $145 \mathrm{pc}$ to compute the luminosity of the star $\left(3.04 L_{\odot}\right)$ and estimate its age $(7.3 \mathrm{Myr})$ based on the Siess et al. (2000) models. We have rescaled the luminosity of the star to correct for the individual distance $\left(d=84.2_{-1.0}^{+1.1} \mathrm{pc}\right)$ that we derived in this paper. This yields a luminosity of $1.03 \mathrm{~L} \odot$ and an age estimate of $24 \mathrm{Myr}$, suggesting that it is much older as indicated in previous studies. More recently, Xing (2010) derived a spectral type of $\mathrm{K} 0$ and measured the $\mathrm{Li}$ equivalent width of only $39 \mathrm{~mA}$. In addition, Xing \& Xing (2012) detected $\mathrm{H} \alpha$ in absorption, and Rebull et al. (2010) found no significant infrared excess for this source based on Spitzer photometry. Altogether, these properties are consistent with V1110 Tau being a young foreground dwarf not related to the Taurus population of YSOs (see also Briceno et al. 1997).

\subsection{1. НP Таи G2}

HP Tau G2 is a weak-line T Tauri star that belongs to a hierarchical triple system with separation of about $10^{\prime \prime}$ from HP Tau G3, which is a tight binary system (see, e.g., Harris et al. 2012; Nguyen et al. 2012). This source has been detected in a total of nine epochs in this work, and it was also observed in the past from 2003 September to 2005 July for projects BL118 and BL124 (see Table 3). We corrected the measured 
positions in these observations before combining them with the more recent GOBELINS data reported in this work. Projects BL118 and BL124 observed J0426+2327 as the main phase calibrator, while the GOBELINS observations use it as a secondary calibrator and $\mathrm{J} 0438+2153$ as the main calibrator. The mean position of J0426+2327 measured between 2003 September and 2005 July is $\alpha=04^{\mathrm{h}} 26^{\mathrm{m}} 55^{\mathrm{s}} .734795, \delta=23^{\circ}$ $27^{\prime} 39$ '. 63371 , and the mean position of J0426+2327 (relative to $\mathrm{J} 0438+2153$ ) in the GOBELINS observations is $\alpha=$ $04^{\mathrm{h}} 26^{\mathrm{m}} 55^{\mathrm{s}} .734757, \delta=23^{\circ} 27^{\prime} 39^{\prime \prime}$. 63403 . Thus, the corresponding offset to correct the measured positions in projects BL118 and BL124 is $\Delta \alpha=-0.000038 \mathrm{~s}$ and $\Delta \delta=0$ ". 00032 . After combining the two data sets, we performed a full fit to solve for the parallax, proper motion, and orbital motion of the HP Tau G2-G3 system (see Tables 5 and 6). The distance that we derive in this paper $(d=162.7 \pm 0.8 \mathrm{pc})$ is in good agreement with the result of $d=161.2 \pm 0.9 \mathrm{pc}$ obtained by Torres et al. (2009) based only on observations collected for projects BL118 and BL124. This confirms that HP Tau G2 is indeed farther than other Taurus stars.

\subsection{V1000 Tau}

V1000 Tau has been detected in seven epochs during our observing campaign, and both components were simultaneously detected in five epochs. On 2017 March 14 (project BL175JW) we measured the highest flux density for the primary component $\left(F_{\nu}=0.96 \pm 0.05 \mathrm{mJy}\right)$. However, both sources appear significantly fainter in the other observations reported in this work, with a flux level below $7 \sigma$, which increases the errors on the individual positions of the sources.

During the calibration process of our observations, we noted that two main calibrators have been used for this source during our observing campaign. Projects BL175AE, BL175AO, BL175HD, BL175IC, BL175JW, and BL175KV observed $\mathrm{J} 0438+2153$ as the main phase calibrator, while projects BL175D2, BL175I7, BL175IZ, BL175JY, and BL175KS used $\mathrm{J} 0429+2724$ in this regard. Thus, we corrected the measured positions in the latter projects before combining the two data sets. J0435+2532 was observed as a secondary calibrator in all projects listed before. The mean position of $\mathrm{J} 0435+2532$ (relative to $\mathrm{J} 0438+2153$ ) in the first data set is $\alpha=$ $04^{\mathrm{h}} 35^{\mathrm{m}} 34^{\mathrm{s}} .582910, \delta=25^{\circ} 32^{\prime} 59^{\prime \prime} .69919$, and the mean position of $\mathrm{J} 0435+2532$ (relative to $\mathrm{J} 0429+2724$ ) in the second data set is $\alpha=04^{\mathrm{h}} 35^{\mathrm{m}} 34^{\mathrm{s}} .582963, \delta=25^{\circ} 32^{\prime} \quad 59^{\prime \prime} 69972$. Thus, we applied an offset of $\Delta \alpha=-0.000053 \mathrm{~s}$ and $\Delta \delta=-0$ ". 00052 to the measured position of the second data set. We applied the same correction to the positions measured for V999 Tau (see Section 4.3) because both sources were observed in the same field and used the same calibrators in all epochs.

We proceeded as follows to calculate the distance to the V1000 Tau system. First, we applied the model with a uniform acceleration to the individual components of the system, but it produced a poor fit to the data. We then considered the model including the orbital motion of the system and solved simultaneously for the proper motion, parallax, and orbital elements. Doing so, we find a distance of $d=136.5_{-2.4}^{+2.5} \mathrm{pc}$. This approach also allowed us to derive the individual masses of the two components of this system $\left(m_{\mathrm{A}}=0.663 \pm\right.$ $0.101 M_{\odot}$ and $\left.m_{\mathrm{B}}=0.550 \pm 0.091 M_{\odot}\right)$. To further investigate our result, we compute the barycenter of the V1000 Tau system using the individual masses derived in this paper and perform a fit including the acceleration terms in our equations. This approach yields a distance estimate of $d=136.4_{-3.2}^{+3.4} \mathrm{pc}$, which is in good agreement with our previous result and confirms our solution despite the low detection threshold.

\subsection{V892 Tau}

V892 Tau is the only source in our sample with a minimum of three detections for which we do not provide a trigonometric parallax. Unlike other stars in our sample, V892 Tau appears as a faint source in our observations, with a detection level of roughly $6 \sigma$ in the best case. V892 Tau is a Herbig Ae/Be star with a low-mass companion (Leinert et al. 1997), and at this stage it is not clear whether the positions measured in this work refer to the same component of the system since they provide a poor astrometric fit.

\subsection{HDE 283572}

HDE 283572 was not included in our target list, but it has been observed by Torres et al. (2007) between 2004 September and 2005 December. We have recalibrated and reanalyzed these data by applying the same methods used for the GOBELINS observations and described throughout this paper. The new distance estimate of $d=129.5_{-0.9}^{+1.0} \mathrm{pc}$ that we derive here is fully consistent with the previous result of $d=$ $128.5 \pm 0.6 \mathrm{pc}$ obtained by Torres et al. (2007).

\section{Discussion}

\subsection{Comparison with Gaia-DR1}

The first step in our analysis to compare the results obtained in this paper with Gaia-DR1 is to build a list of Taurus stars that have been previously identified in the literature. In a recent study, Joncour et al. (2017) published an updated census with 338 stars (and stellar systems) in this region. We add V1201 Tau, HD 283641, and V1110 Tau to their list, which have been investigated in this work and were not included in their compilation.

The Gaia satellite observed 204 stars in our list of known YSOs in the Taurus region, but the Tycho-Gaia Astrometric Solution (TGAS; Lindegren et al. 2016) catalog from GaiaDR1 provides trigonometric parallaxes for only 18 stars (and stellar systems). Eight stars are in common with our sample of 18 stars with measured trigonometric parallaxes (see Table 5), including V1110 Tau, which is more likely a foreground dwarf (see Section 4.10). Figure 7 illustrates the comparison of our results with Gaia-DR1. The rms and mean difference between the trigonometric parallaxes derived in both projects are, respectively, 0.38 and -0.15 mas (in the sense, "GOBELINS" minus "TGAS"). These numbers are smaller than the mean error of $\sigma_{\pi}=0.46$ mas on the trigonometric parallaxes from Gaia-DR1 in the Taurus region. Thus, our results are in good agreement with Gaia-DR1, but the trigonometric parallaxes derived in this work are more precise than the ones given in the TGAS catalog. For example, we measured a trigonometric parallax of $\pi=7.751 \pm 0.027$ mas for HD 283518 that lies exactly on the equality line (see Figure 7) and is more precise by almost one order of magnitude than the result of $\pi=7.78 \pm 0.29$ mas delivered by Gaia-DR1. This confirms the state-of-the-art accuracy and precision that can be obtained from VLBI astrometry and the good complementarity with the Gaia space mission. 


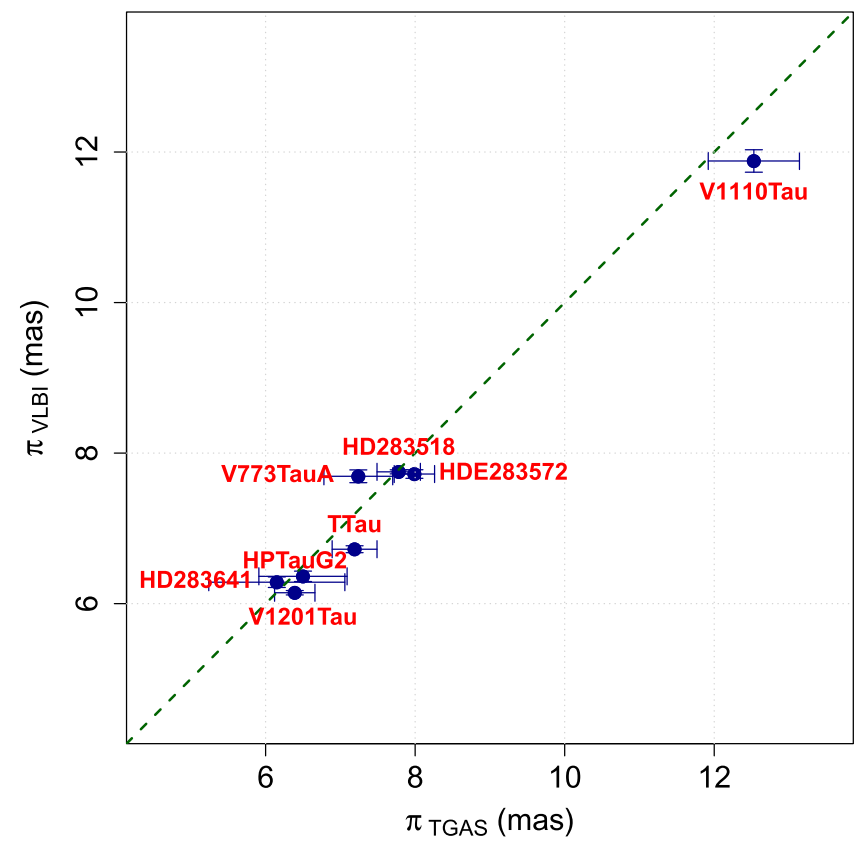

Figure 7. Comparison of the trigonometric parallaxes derived in this paper with the results delivered by the TGAS catalog from Gaia-DR1 for the stars in common. The green dashed line indicates perfect correlation of both data sets.

Two points regarding the comparison of our results with Gaia-DR1 are worth mentioning here. First, the trigonometric parallaxes in the TGAS catalog are affected by a systematic error of about 0.30 mas, depending, for example, on the position and color of the stars (Lindegren et al. 2016). Thus, we added the value of 0.30 mas quadratically to the formal errors given in the TGAS catalog. The second and potentially more serious problem is that most of our sources in common with Gaia-DR1 are binaries (or multiple systems). In this context, it is important to mention that all sources in the TGAS catalog were modeled as single stars, so that the orbital motion in binaries is neglected. This explains the most discrepant results obtained for some sources in Figure 7 (e.g., T Tau Sb and V773 Tau A), where this analysis is of ultimate importance and can only be applied with long-term monitoring of the system under investigation. For the reasons mentioned above, we consider the trigonometric parallaxes from the GOBELINS project to be more accurate and precise than the ones given in Gaia-DR1 for the targets in common.

\subsection{Distance and Spatial Velocity of Taurus Stars}

The effective sample of stars that we use in the forthcoming analysis to discuss the distance and kinematics of the Taurus star-forming region consists of 26 stars (or stellar systems). It includes all stars (or stellar systems) listed in Table 5 (excluding V1110 Tau) and the additional 10 sources with trigonometric parallaxes from Gaia-DR1 (see Section 5.1). V1110 Tau is not included in this discussion for the reasons presented in Section 4.10. We use the proper motions and trigonometric parallaxes derived in this work from VLBI observations, and we take the results from the TGAS catalog to complement our sample for stars that were not included in our observing campaign. In the case of V1096 Tau we use the weighted mean parallax and proper motion of the two components given in Section 4.1, which provide a better solution for the system than the individual values listed in
Table 5. Figure 8 summarizes the trigonometric parallaxes of the stars in our sample, and Figures 9-12 illustrate the structure in and around the various star-forming clouds investigated in our analysis based on the extinction maps from Dobashi et al. (2005).

We also searched the literature for the radial velocity of the stars in our sample using the data mining tools available in the CDS databases (Wenger et al. 2000). Our search for radial velocities is based on Hartmann et al. (1986), Herbig \& Bell (1988), Gontcharov (2006), Kharchenko et al. (2007), and Nguyen et al. (2012). The properties of GOBELINS and GaiaDR1 stars in our sample are collectively listed in Table 8. Then, we converted the observed trigonometric parallaxes into distances and used the radial velocities to calculate the threedimensional Galactic spatial velocities from the procedure described by Johnson \& Soderblom (1987). In Table 9 we present the $U V W$ spatial velocity and the peculiar velocity of individual stars in our sample after correcting for the velocity of the Sun with respect to the local standard of rest (LSR). For this correction we use the solar motion obtained by Schönrich et al. (2010). We also present in Table 9 the radial velocities of the stars converted to the LSR, which will be used in the forthcoming discussion to compare with the velocity field of the $\mathrm{CO}$ molecular gas in this region produced by the Five College Radio Astronomy Observatory (FCRAO). In this case we used the older IAU standard solar motion to convert the radial velocities to the LSR for consistency with the FCRAO maps (see Jackson et al. 2006). In the following we comment on the distance and kinematics of the individual clouds of the Taurus complex. The properties of the various star-forming clouds discussed below are also summarized in Table 10.

\subsubsection{Lynds 1495}

Lynds 1495 (L1495; Lynds 1962) is the main star-forming site of the Taurus complex and the most important structure to discuss in this work, because it contains about $40 \%$ of the stars in our sample. Schmalzl et al. (2010) divided it into five clumps (B211, B213, B216, B217, and B218), which form the filament projected on the plane of the sky, and the central part B10. We note that V1023 Tau, HD 283518, and 2MASS J04182909 +2826191 are located in the northern part of B10 (see Figure 9). The weighted mean parallax of these sources is $\pi=7.724 \pm 0.021$ mas, which is consistent with a distance estimate of $d=129.5_{-0.3}^{+0.4} \mathrm{pc}$. On the other hand, V1096 Tau, V1098 Tau, and V773 Tau are projected toward the southern part of the B10 clump with a weighted mean parallax of $\pi=7.727 \pm 0.081 \mathrm{mas}$ and distance of $d=129.4_{-1.3}^{+1.4} \mathrm{pc}$. Thus, we conclude that both substructures are located at the same distance. As discussed in Section 4, the trigonometric parallaxes obtained in this paper for V1096 Tau, V1098 Tau, and 2MASS J04182909+28261 require further improvement, but based on the current results, we find no evidence of significant depth effects within the B10 clump of the L1495 cloud. The weighted mean parallax of the six stars mentioned before is $\pi=7.724 \pm 0.020$ mas. This is consistent with a distance estimate of $d=129.5_{-0.3}^{+0.3} \mathrm{pc}$, which is the most precise and accurate present-day distance determination of L1495.

Figure 9 reveals three stars in our sample (HDE 283572, RY Tau, and BPTau) in the outskirts of L1495. We note that the trigonometric parallaxes of HDE 283572 $(\pi=7.722 \pm 0.057$ mas $)$ and BP Tau $(\pi=7.900 \pm 0.492$ mas $)$ are consistent with the results obtained in this paper for the other 


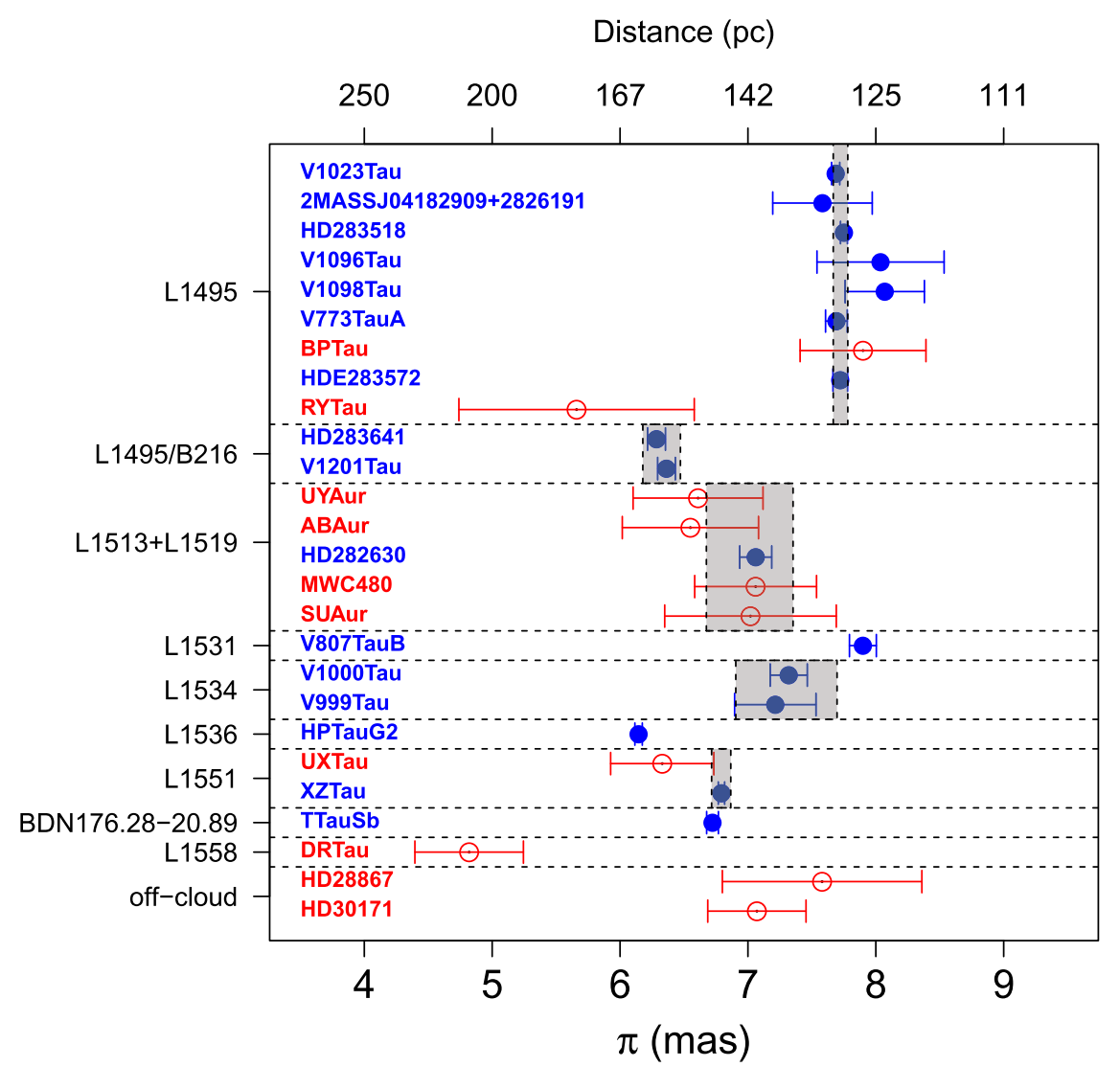

Figure 8. Summary of the trigonometric parallax measurements for Taurus stars. The sources are grouped according to the star-forming cloud to which they belong. Filled and open symbols indicate, respectively, VLBI trigonometric parallaxes obtained in this paper and TGAS results from Gaia-DR1. The gray vertical bars indicate the weighted mean parallax (at the $3 \sigma$ level) of the clouds with more than one member in our sample to illustrate the depth effects.

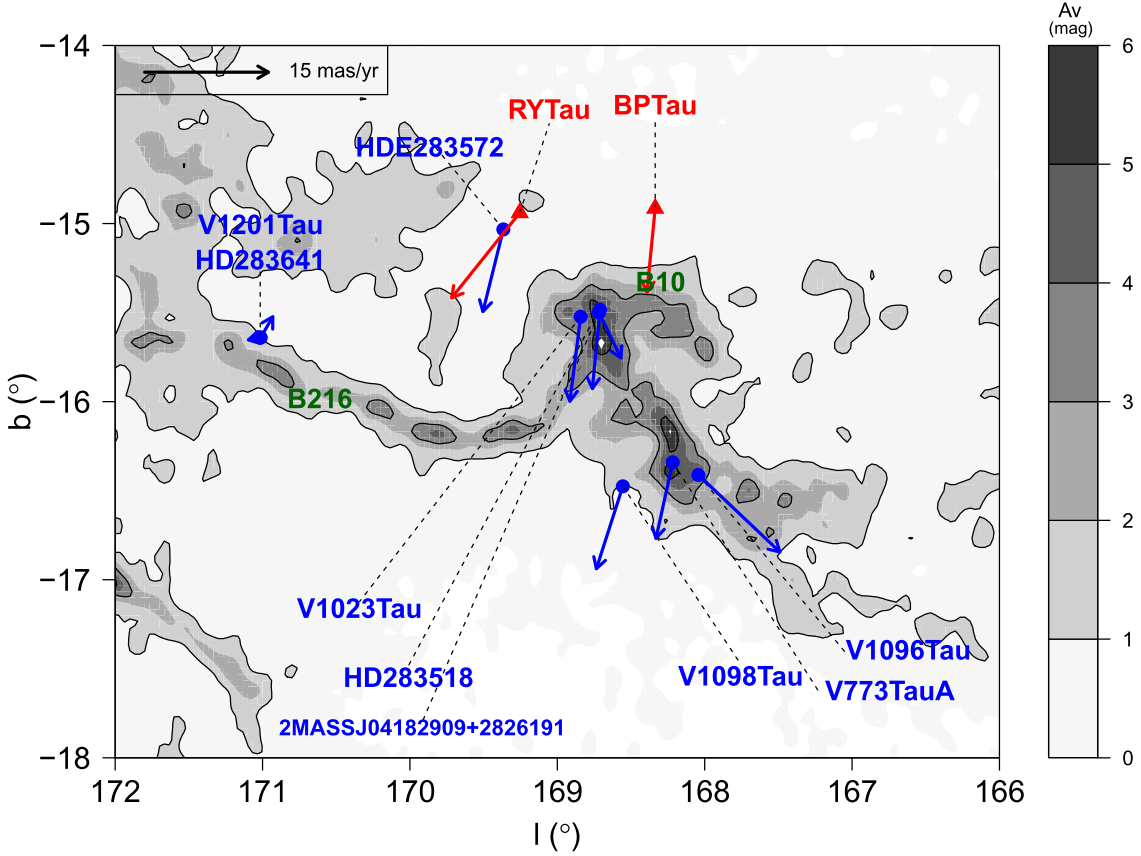

Figure 9. Structure of the L1495 cloud $\left(d=129.5_{-0.3}^{+0.3} \mathrm{pc}\right)$ overlaid on the extinction map from Dobashi et al. (2005). Blue circles and red triangles denote, respectively, the stars with VLBI and TGAS trigonometric parallax. The vectors indicate the stellar proper motions from Table 8 converted to the Galactic reference system and corrected for the solar motion (Schönrich et al. 2010) using the formalism described by Abad \& Vieira (2005).

stars in the B10 clump. However, the trigonometric parallax of RY Tau given in the TGAS catalog $(\pi=5.660 \pm 0.920$ mas) is in obvious disagreement with the other stars, suggesting either a problem with the result delivered by Gaia-DR1 (e.g., noncorrected binarity or low precision/accuracy) or that RY Tau is indeed not related to the L1495 cloud. Moreover, we note that its 
Table 8

Proper Motions, Trigonometric Parallaxes, Distances, and Radial Velocities of the GOBELINS-Gaia Sample in Taurus

\begin{tabular}{|c|c|c|c|c|c|c|c|c|}
\hline Star & 2MASS Identifier & $\begin{array}{c}\mu_{\alpha} \cos \delta \\
\left({\left.\text { mas } y r^{-1}\right)}^{-1}\right.\end{array}$ & $\begin{array}{c}\mu_{\delta} \\
\left(\operatorname{mas~yr}^{-1}\right)\end{array}$ & $\begin{array}{c}\pi \\
\text { (mas) }\end{array}$ & $\begin{array}{c}d \\
(\mathrm{pc})\end{array}$ & Reference & $\begin{array}{c}V_{r} \\
\left(\mathrm{~km} \mathrm{~s}^{-1}\right)\end{array}$ & Reference \\
\hline V1096 Tau & J04132722+2816247 & $2.612 \pm 0.691$ & $-17.372 \pm 0.676$ & $8.037 \pm 0.497$ & $124.4_{-7.2}^{+8.2}$ & This work & $12.00 \pm 5.00$ & 1 \\
\hline V773 Tau A & $\mathrm{J} 04141291+2812124$ & $10.253 \pm 0.843$ & $-25.119 \pm 0.301$ & $7.692 \pm 0.085$ & $130.0_{-1.4}^{+1.5}$ & This work & $16.00 \pm 2.50$ & 2 \\
\hline HD 283518 & $\mathrm{~J} 04183110+2827162$ & $8.703 \pm 0.017$ & $-24.985 \pm 0.020$ & $7.751 \pm 0.027$ & $129.0_{-0.4}^{+0.5}$ & This work & $19.90 \pm 0.30$ & 3 \\
\hline V1023 Tau & $\mathrm{J} 04184703+2820073$ & $8.371 \pm 0.020$ & $-25.490 \pm 0.020$ & $7.686 \pm 0.032$ & $130.1_{-0.5}^{+0.5}$ & This work & $15.00 \pm 1.70$ & 4 \\
\hline BP Tau & J04191583+2906269 & $8.683 \pm 0.355$ & $-25.846 \pm 0.239$ & $7.900 \pm 0.492$ & $126.6_{-7.4}^{+8.4}$ & Gaia-DR1 & $15.24 \pm 0.04$ & 3 \\
\hline RY Tau & $\mathrm{J} 04215740+2826355$ & $9.100 \pm 0.140$ & $-25.859 \pm 0.091$ & $5.660 \pm 0.920$ & $176.7_{-24.7}^{+34.3}$ & Gaia-DR1 & $24.30 \pm 1.90$ & 2 \\
\hline HDE 283572 & $\mathrm{~J} 04215884+2818066$ & $8.853 \pm 0.096$ & $-26.491 \pm 0.113$ & $7.722 \pm 0.057$ & $129.5_{-0.9}^{+1.0}$ & This work & $14.22 \pm 0.08$ & 3 \\
\hline T Tau Sb & J04215943+1932063 & $6.790 \pm 0.432$ & $-11.131 \pm 0.444$ & $6.723 \pm 0.046$ & $148.7_{-1.0}^{+1.0}$ & This work & $19.23 \pm 0.02$ & 3 \\
\hline HD 283641 & J04244904+2643104 & $10.913 \pm 0.037$ & $-16.772 \pm 0.044$ & $6.285 \pm 0.070$ & $159.1_{-1.8}^{+1.8}$ & This work & $16.23 \pm 0.04$ & 3 \\
\hline UX Tau & $\mathrm{J} 04300399+1813493$ & $12.641 \pm 0.435$ & $-17.552 \pm 0.257$ & $6.330 \pm 0.404$ & $158.0_{-9.5}^{+10.8}$ & Gaia-DR1 & $15.45 \pm 0.02$ & 3 \\
\hline XZ Tau & $\mathrm{J} 04314007+1813571$ & $10.858 \pm 0.027$ & $-16.264 \pm 0.060$ & $6.793 \pm 0.025$ & $147.2_{-0.5}^{+0.5}$ & This work & $18.30 \pm 0.04$ & 3 \\
\hline V807 Tau B & $\mathrm{J} 04330664+2409549$ & $8.573 \pm 0.068$ & $-28.774 \pm 0.201$ & $7.899 \pm 0.105$ & $126.6_{-1.7}^{+1.7}$ & This work & $16.85 \pm 0.03$ & 3 \\
\hline HD 28867 & J04333297+1801004 & $12.017 \pm 0.047$ & $-18.635 \pm 0.030$ & $7.580 \pm 0.780$ & $131.9_{-12.3}^{+15.1}$ & Gaia-DR1 & $-14.70 \pm 7.40$ & 5 \\
\hline HP Tau G2 & $\mathrm{J} 04355415+2254134$ & $11.248 \pm 0.022$ & $-15.686 \pm 0.013$ & $6.145 \pm 0.029$ & $162.7_{-0.8}^{+0.8}$ & This work & $16.60 \pm 1.00$ & 3 \\
\hline V999 Tau & $\mathrm{J} 04420548+2522562$ & $9.533 \pm 0.218$ & $-15.684 \pm 0.198$ & $6.972 \pm 0.197$ & $143.4_{-3.9}^{+4.2}$ & This work & $14.70 \pm 2.00$ & 1 \\
\hline HD 30171 & J04455129+1555496 & $9.824 \pm 1.675$ & $-24.288 \pm 0.994$ & $7.070 \pm 0.384$ & $141.4_{-7.3}^{+8.1}$ & Gaia-DR1 & $21.13 \pm 0.17$ & 3 \\
\hline DR Tau & $\mathrm{J} 04470620+1658428$ & $1.372 \pm 2.747$ & $-11.225 \pm 1.785$ & $4.820 \pm 0.424$ & $207.5_{-16.8}^{+20.0}$ & Gaia-DR1 & $21.10 \pm 0.04$ & 3 \\
\hline UY Aur & $\mathrm{J} 04514737+3047134$ & $6.099 \pm 2.824$ & $-27.027 \pm 1.840$ & $6.610 \pm 0.508$ & $151.3_{-10.8}^{+12.6}$ & Gaia-DR1 & $13.92 \pm 0.07$ & 3 \\
\hline HD 282630 & $\mathrm{~J} 04553695+3017553$ & $3.897 \pm 0.113$ & $-24.210 \pm 0.132$ & $7.061 \pm 0.125$ & $141.6_{-2.5}^{+2.6}$ & This work & $13.58 \pm 0.01$ & 3 \\
\hline $\mathrm{AB}$ Aur & $\mathrm{J} 04554582+3033043$ & $3.889 \pm 0.056$ & $-24.050 \pm 0.039$ & $6.550 \pm 0.533$ & $152.7_{-11.5}^{+13.5}$ & Gaia-DR1 & $8.90 \pm 0.90$ & 2 \\
\hline SU Aur & $\mathrm{J} 04555938+3034015$ & $3.857 \pm 0.126$ & $-24.367 \pm 0.088$ & $7.020 \pm 0.671$ & $142.5_{-12.4}^{+15.1}$ & Gaia-DR1 & $14.26 \pm 0.05$ & 3 \\
\hline V1098 Tau & $\mathrm{J} 04144797+2752346$ & $11.148 \pm 0.175$ & $-27.327 \pm 0.172$ & $8.070 \pm 0.310$ & $123.9_{-4.6}^{+5.0}$ & This work & $\cdots$ & $\cdots$ \\
\hline 2MASS J04182909+2826191 & J04182909+2826191 & $8.384 \pm 0.195$ & $-19.627 \pm 0.217$ & $7.583 \pm 0.389$ & $131.9_{-6.4}^{+7.1}$ & This work & $\cdots$ & $\cdots$ \\
\hline V1201 Tau & $\mathrm{J} 04244815+2643161$ & $10.839 \pm 0.050$ & $-13.235 \pm 0.058$ & $6.363 \pm 0.069$ & $157.2_{-1.7}^{+1.7}$ & This work & $\cdots$ & $\cdots$ \\
\hline V1000 Tau & $\mathrm{J} 04420732+2523032$ & $6.010 \pm 0.235$ & $-17.720 \pm 0.159$ & $7.324 \pm 0.132$ & $136.5_{-2.4}^{+2.5}$ & This work & $\ldots$ & $\cdots$ \\
\hline MWC 480 & $\mathrm{~J} 04584626+2950370$ & $4.790 \pm 0.081$ & $-25.044 \pm 0.049$ & $7.060 \pm 0.476$ & $141.6_{-9.0}^{+10.2}$ & Gaia-DR1 & $\cdots$ & $\cdots$ \\
\hline
\end{tabular}

Note. References for radial velocities: (1) Herbig \& Bell 1988; (2) Gontcharov 2006; (3) Nguyen et al. 2012; (4) Hartmann et al. 1986; (5) Kharchenko et al. 2007. 
Table 9

Spatial Velocity for Taurus Stars with Measured Trigonometric Parallaxes and Radial Velocities

\begin{tabular}{|c|c|c|c|c|c|c|c|c|c|c|}
\hline Star & $\begin{array}{l}\text { 2MASS Identifier } \\
\text { (2) }\end{array}$ & $\begin{array}{c}U \\
\left(\mathrm{~km} \mathrm{~s}^{-1}\right) \\
(3)\end{array}$ & $\begin{array}{c}V \\
\left(\mathrm{~km} \mathrm{~s}^{-1}\right) \\
(4)\end{array}$ & $\begin{array}{c}W \\
\left(\mathrm{~km} \mathrm{~s}^{-1}\right) \\
(5)\end{array}$ & $\begin{array}{c}V_{\text {space }} \\
\left(\mathrm{km} \mathrm{s}^{-1}\right) \\
(6)\end{array}$ & $\begin{array}{c}u \\
\left(\mathrm{~km} \mathrm{~s}^{-1}\right) \\
(7)\end{array}$ & $\begin{array}{c}\begin{array}{c}v \\
\left(\mathrm{~km} \mathrm{~s}^{-1}\right)\end{array} \\
(8)\end{array}$ & 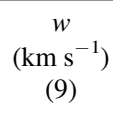 & $\begin{array}{c}V_{\mathrm{pec}} \\
\left(\mathrm{km} \mathrm{s}^{-1}\right) \\
(10)\end{array}$ & $\begin{array}{c}V_{r}^{\mathrm{LSR}} \\
\left(\mathrm{km} \mathrm{s}^{-1}\right) \\
(11)\end{array}$ \\
\hline V1096 Tau & J04132722+2816247 & $-11.4_{-4.9}^{+4.9}$ & $-6.3_{-2.1}^{+2.0}$ & $-9.1_{-2.4}^{+2.4}$ & $15.9_{-3.9}^{+3.9}$ & $-0.3_{-5.0}^{+5.0}$ & $6.0_{-2.2}^{+2.0}$ & $-1.8_{-2.5}^{+2.4}$ & $6.2_{-2.2}^{+2.1}$ & $6.29 \pm 5.00$ \\
\hline V773 Tau A & $\mathrm{J} 04141291+2812124$ & $-16.5_{-2.6}^{+2.6}$ & $-12.5_{-1.1}^{+1.1}$ & $-10.3_{-1.3}^{+1.3}$ & $23.1_{-2.0}^{+2.0}$ & $-5.4_{-2.7}^{+2.7}$ & $-0.3_{-1.2}^{+1.2}$ & $-3.1_{-1.4}^{+1.4}$ & $6.2_{-2.4}^{+2.4}$ & $10.27 \pm 2.50$ \\
\hline HD 283518 & $\mathrm{~J} 04183110+2827162$ & $-20.0_{-0.3}^{+0.3}$ & $-11.2_{-0.1}^{+0.1}$ & $-11.5_{-0.1}^{+0.1}$ & $25.6_{-0.2}^{+0.2}$ & $-8.9_{-0.8}^{+0.8}$ & $1.1_{-0.5}^{+0.5}$ & $-4.3_{-0.4}^{+0.4}$ & $9.9_{-0.7}^{+0.7}$ & $14.13 \pm 0.30$ \\
\hline V1023 Tau & $\mathrm{J} 04184703+2820073$ & $-15.3_{-1.6}^{+1.6}$ & $-12.4_{-0.4}^{+0.4}$ & $-10.6_{-0.5}^{+0.5}$ & $22.3_{-1.2}^{+1.2}$ & $-4.2_{-1.8}^{+1.8}$ & $-0.1_{-0.6}^{+0.6}$ & $-3.4_{-0.6}^{+0.6}$ & $5.4_{-1.4}^{+1.4}$ & $9.20 \pm 1.70$ \\
\hline BP Tau & J04191583+2906269 & $-15.7_{-0.2}^{+0.2}$ & $-12.0_{-1.3}^{+1.1}$ & $-10.4_{-1.2}^{+1.1}$ & $22.4_{-0.9}^{+0.8}$ & $-4.6_{-0.8}^{+0.7}$ & $0.2_{-1.3}^{+1.2}$ & $-3.2_{-1.2}^{+1.2}$ & $5.6_{-0.9}^{+0.9}$ & $9.57 \pm 0.04$ \\
\hline RY Tau & $\mathrm{J} 04215740+2826355$ & $-24.7_{-2.4}^{+2.3}$ & $-16.9_{-4.6}^{+3.4}$ & $-14.8_{-4.1}^{+3.7}$ & $33.4_{-3.5}^{+2.9}$ & $-13.6_{-2.5}^{+2.4}$ & $-4.7_{-4.6}^{+3.4}$ & $-7.6_{-4.1}^{+3.7}$ & $16.3_{-3.2}^{+2.8}$ & $18.46 \pm 1.90$ \\
\hline HDE 283572 & J04215884+2818066 & $-14.6_{-0.1}^{+0.1}$ & $-13.2_{-0.2}^{+0.2}$ & $-10.4_{-0.2}^{+0.2}$ & $22.3_{-0.2}^{+0.2}$ & $-3.5_{-0.8}^{+0.7}$ & $-1.0_{-0.5}^{+0.5}$ & $-3.1_{-0.4}^{+0.4}$ & $4.8_{-0.6}^{+0.6}$ & $8.36 \pm 0.08$ \\
\hline T Tau Sb & J04215943+1932063 & $-18.0_{-0.2}^{+0.2}$ & $-8.0_{-0.5}^{+0.5}$ & $-8.1_{-0.5}^{+0.5}$ & $21.3_{-0.3}^{+0.3}$ & $-6.9_{-0.8}^{+0.7}$ & $4.2_{-0.7}^{+0.7}$ & $-0.8_{-0.6}^{+0.6}$ & $8.2_{-0.7}^{+0.7}$ & $11.83 \pm 0.02$ \\
\hline HD 283641 & J04244904+2643104 & $-17.1_{-0.1}^{+0.1}$ & $-12.5_{-0.2}^{+0.2}$ & $-6.5_{-0.2}^{+0.2}$ & $22.2_{-0.2}^{+0.1}$ & $-6.0_{-0.8}^{+0.7}$ & $-0.2_{-0.5}^{+0.5}$ & $0.8_{-0.4}^{+0.4}$ & $6.1_{-0.8}^{+0.7}$ & $10.02 \pm 0.04$ \\
\hline UX Tau & J04300399+1813493 & $-14.5_{-0.5}^{+0.5}$ & $-15.9_{-1.5}^{+1.3}$ & $-6.1_{-1.3}^{+1.3}$ & $22.4_{-1.2}^{+1.0}$ & $-3.4_{-0.9}^{+0.8}$ & $-3.7_{-1.6}^{+1.4}$ & $1.1_{-1.3}^{+1.4}$ & $5.1_{-1.3}^{+1.2}$ & $7.68 \pm 0.02$ \\
\hline XZ Tau & J04314007+1813571 & $-17.0_{-0.1}^{+0.1}$ & $-13.4_{-0.1}^{+0.1}$ & $-7.3_{-0.1}^{+0.1}$ & $22.8_{-0.1}^{+0.1}$ & $-5.9_{-0.8}^{+0.7}$ & $-1.1_{-0.5}^{+0.5}$ & $0.0_{-0.4}^{+0.4}$ & $6.0_{-0.7}^{+0.7}$ & $10.50 \pm 0.04$ \\
\hline V807 Tau B & J04330664+2409549 & $-15.8_{-0.1}^{+0.1}$ & $-15.1_{-0.3}^{+0.3}$ & $-11.4_{-0.3}^{+0.3}$ & $24.7_{-0.3}^{+0.3}$ & $-4.7_{-0.8}^{+0.7}$ & $-2.9_{-0.6}^{+0.6}$ & $-4.2_{-0.5}^{+0.5}$ & $6.9_{-0.6}^{+0.6}$ & $10.04 \pm 0.03$ \\
\hline HD 28867 & J04333297+1801004 & $14.3_{-7.4}^{+7.5}$ & $-13.9_{-1.7}^{+1.4}$ & $3.5_{-3.8}^{+3.7}$ & $20.2_{-5.4}^{+5.4}$ & $25.4_{-7.5}^{+7.5}$ & $-1.6_{-1.7}^{+1.4}$ & $10.7_{-3.8}^{+3.8}$ & $27.6_{-7.0}^{+7.0}$ & $-22.57 \pm 7.40$ \\
\hline HP Tau G2 & $\mathrm{J} 04355415+2254134$ & $-16.7_{-1.0}^{+1.0}$ & $-13.8_{-0.2}^{+0.2}$ & $-5.5_{-0.4}^{+0.4}$ & $22.3_{-0.7}^{+0.7}$ & $-5.6_{-1.2}^{+1.2}$ & $-1.5_{-0.5}^{+0.5}$ & $1.8_{-0.5}^{+0.5}$ & $6.1_{-1.2}^{+1.1}$ & $9.52 \pm 1.00$ \\
\hline V999 Tau & $\mathrm{J} 04420548+2522562$ & $-15.0_{-2.1}^{+2.0}$ & $-11.1_{-0.7}^{+0.7}$ & $-5.0_{-1.0}^{+1.0}$ & $19.3_{-1.7}^{+1.7}$ & $-3.9_{-2.2}^{+2.2}$ & $1.1_{-0.9}^{+0.8}$ & $2.3_{-1.0}^{+1.0}$ & $4.6_{-1.9}^{+1.9}$ & $7.96 \pm 2.00$ \\
\hline HD 30171 & J04455129+1555496 & $-17.7_{-0.8}^{+0.8}$ & $-18.1_{-2.3}^{+2.0}$ & $-10.8_{-2.1}^{+2.1}$ & $27.5_{-1.8}^{+1.7}$ & $-6.6_{-1.1}^{+1.1}$ & $-5.8_{-2.3}^{+2.1}$ & $-3.5_{-2.1}^{+2.1}$ & $9.5_{-1.8}^{+1.7}$ & $12.71 \pm 0.17$ \\
\hline DR Tau & $\mathrm{J} 04470620+1658428$ & $-18.0_{-1.2}^{+1.3}$ & $-10.5_{-4.3}^{+3.7}$ & $-11.5_{-3.9}^{+3.8}$ & $23.9_{-2.8}^{+2.6}$ & $-6.9_{-1.4}^{+1.4}$ & $1.7_{-4.3}^{+3.7}$ & $-4.3_{-3.9}^{+3.8}$ & $8.3_{-2.5}^{+2.4}$ & $12.83 \pm 0.04$ \\
\hline UY Aur & $\mathrm{J} 04514737+3047134$ & $-14.9_{-0.6}^{+0.5}$ & $-16.0_{-3.9}^{+3.4}$ & $-10.5_{-3.6}^{+3.6}$ & $24.3_{-3.1}^{+2.7}$ & $-3.8_{-1.0}^{+0.9}$ & $-3.7_{-4.0}^{+3.4}$ & $-3.3_{-3.6}^{+3.6}$ & $6.3_{-3.1}^{+2.8}$ & $8.05 \pm 0.07$ \\
\hline HD 282630 & $\mathrm{~J} 04553695+3017553$ & $-14.1_{-0.1}^{+0.1}$ & $-12.8_{-0.4}^{+0.4}$ & $-9.7_{-0.3}^{+0.3}$ & $21.3_{-0.3}^{+0.3}$ & $-3.0_{-0.8}^{+0.7}$ & $-0.6_{-0.6}^{+0.6}$ & $-2.4_{-0.5}^{+0.5}$ & $3.9_{-0.7}^{+0.6}$ & $7.57 \pm 0.01$ \\
\hline $\mathrm{AB}$ Aur & $\mathrm{J} 04554582+3033043$ & $-9.6_{-1.0}^{+1.0}$ & $-14.4_{-1.5}^{+1.3}$ & $-9.6_{-1.3}^{+1.2}$ & $19.7_{-1.4}^{+1.2}$ & $1.5_{-1.2}^{+1.2}$ & $-2.1_{-1.6}^{+1.4}$ & $-2.3_{-1.3}^{+1.2}$ & $3.5_{-1.4}^{+1.3}$ & $2.94 \pm 0.90$ \\
\hline SU Aur & $\mathrm{J} 04555938+3034015$ & $-14.8_{-0.1}^{+0.1}$ & $-12.8_{-1.7}^{+1.4}$ & $-9.8_{-1.3}^{+1.2}$ & $21.9_{-1.1}^{+1.0}$ & $-3.7_{-0.8}^{+0.7}$ & $-0.6_{-1.7}^{+1.4}$ & $-2.6_{-1.4}^{+1.3}$ & $4.6_{-1.0}^{+0.9}$ & $8.30 \pm 0.05$ \\
\hline
\end{tabular}

Note. Columns (3)-(6) provide the stellar spatial velocity not corrected for the solar motion. The peculiar velocities of the stars after correcting for the velocity of the Sun with respect to the LSR are given in Columns (7)-(10). The radial velocities of the stars (see Table 8) converted to the LSR are given in Column (11).

Table 10

Distance and Spatial Velocity of the Various Clouds in Taurus

\begin{tabular}{|c|c|c|c|c|c|c|c|c|}
\hline Cloud & $N_{1}$ & $N_{2}$ & $\begin{array}{c}\pi \\
\text { (mas) }\end{array}$ & $\begin{array}{c}d \\
(\mathrm{pc})\end{array}$ & $\begin{array}{c}U \\
\left(\mathrm{~km} \mathrm{~s}^{-1}\right)\end{array}$ & $\begin{array}{c}V \\
\left(\mathrm{~km} \mathrm{~s}^{-1}\right)\end{array}$ & $\begin{array}{c}W \\
\left(\mathrm{~km} \mathrm{~s}^{-1}\right)\end{array}$ & $\begin{array}{c}V_{\text {space }} \\
\left(\mathrm{km} \mathrm{s}^{-1}\right)\end{array}$ \\
\hline L1495 & 8 & 6 & $7.724 \pm 0.019$ & $129.5_{-0.3}^{+0.3}$ & $-15.4 \pm 0.1$ & $-11.7 \pm 0.1$ & $-11.1 \pm 0.1$ & $23.4 \pm 0.1$ \\
\hline L1495 (B216) & 2 & 1 & $6.325 \pm 0.049$ & $158.1_{-1.2}^{+1.2}$ & $-17.1 \pm 0.1$ & $-12.5 \pm 0.2$ & $-6.5 \pm 0.2$ & $22.2 \pm 0.1$ \\
\hline L1513 & 1 & 1 & $6.610 \pm 0.508$ & $151_{-11}^{+13}$ & $-14.9 \pm 0.6$ & $-16.0 \pm 3.7$ & $-10.5 \pm 3.6$ & $24.3 \pm 2.9$ \\
\hline L1519 & 4 & 3 & $7.035 \pm 0.116$ & $142.1_{-2.3}^{+2.4}$ & $-14.1 \pm 0.1$ & $-12.9 \pm 0.4$ & $-9.7 \pm 0.3$ & $21.3 \pm 0.3$ \\
\hline L1531 & 1 & 1 & $7.899 \pm 0.105$ & $126.6_{-1.7}^{+1.7}$ & $-15.8 \pm 0.1$ & $-15.1 \pm 0.3$ & $-11.4 \pm 0.3$ & $24.7 \pm 0.3$ \\
\hline L1551 & 2 & 2 & $6.791 \pm 0.025$ & $147.3_{-0.5}^{+0.5}$ & $-17.0 \pm 0.1$ & $-13.4 \pm 0.1$ & $-7.2 \pm 0.1$ & $22.8 \pm 0.1$ \\
\hline L1558 & 1 & 1 & $4.820 \pm 0.424$ & $208_{-17}^{+20}$ & $-18.0 \pm 1.2$ & $-10.5 \pm 4.0$ & $-11.5 \pm 3.8$ & $23.9 \pm 2.7$ \\
\hline Taurus (all stars) & 23 & 18 & $7.054 \pm 0.012$ & $141.8_{-0.2}^{+0.2}$ & $-15.2 \pm 0.1$ & $-12.8 \pm 0.1$ & $-8.7 \pm 0.1$ & \pm 0.1 \\
\hline
\end{tabular}

Note. We provide for each subgroup the number of stars with known trigonometric parallax $\left(N_{1}\right)$ and radial velocity $\left(N_{2}\right)$, the weighted mean parallax with the corresponding distance, and the weighted mean spatial velocity.

radial velocity exceeds the mean radial velocity of the other stars in this region by about $9 \mathrm{~km} \mathrm{~s}^{-1}$ (see Table 8). The revised weighted mean parallax of L1495 (including HDE 283572 and BP Tau) is $\pi=7.724 \pm 0.019$ mas and yields a distance of $d=129.5_{-0.3}^{+0.3} \mathrm{pc}$, confirming our previous result.

One interesting finding of our analysis is that V1201 Tau and HD 283641, which are projected toward the B216 clump in the filamentary structure of L1495, are located at a different distance as compared to the other stars in L1495. The mean parallax of these two sources is $\pi=6.325 \pm 0.049$ mas and yields a distance of $d=158.1_{-1.2}^{+1.2} \mathrm{pc}$. This distance estimate differs by almost $30 \mathrm{pc}$ from the result mentioned before and reveals the existence of important depth effects between the central part of the cloud and the filament. In addition, the spatial velocity that we derive for HD 283641 also suggests that both structures exhibit different kinematic properties (see Table 10).

\subsubsection{Lynds 1513 and 1519}

UY Aur is projected toward the Lynds 1513 cloud (L1513; Lynds 1962) with a trigonometric parallax of $\pi=6.610 \pm$ 0.508 mas. This result is in good agreement (at the $1 \sigma$ level) with the trigonometric parallaxes of $\mathrm{AB}$ Aur $(\pi=6.550 \pm$ 0.532 mas) and SU Aur $(\pi=7.020 \pm 0.671$ mas $)$, which are 


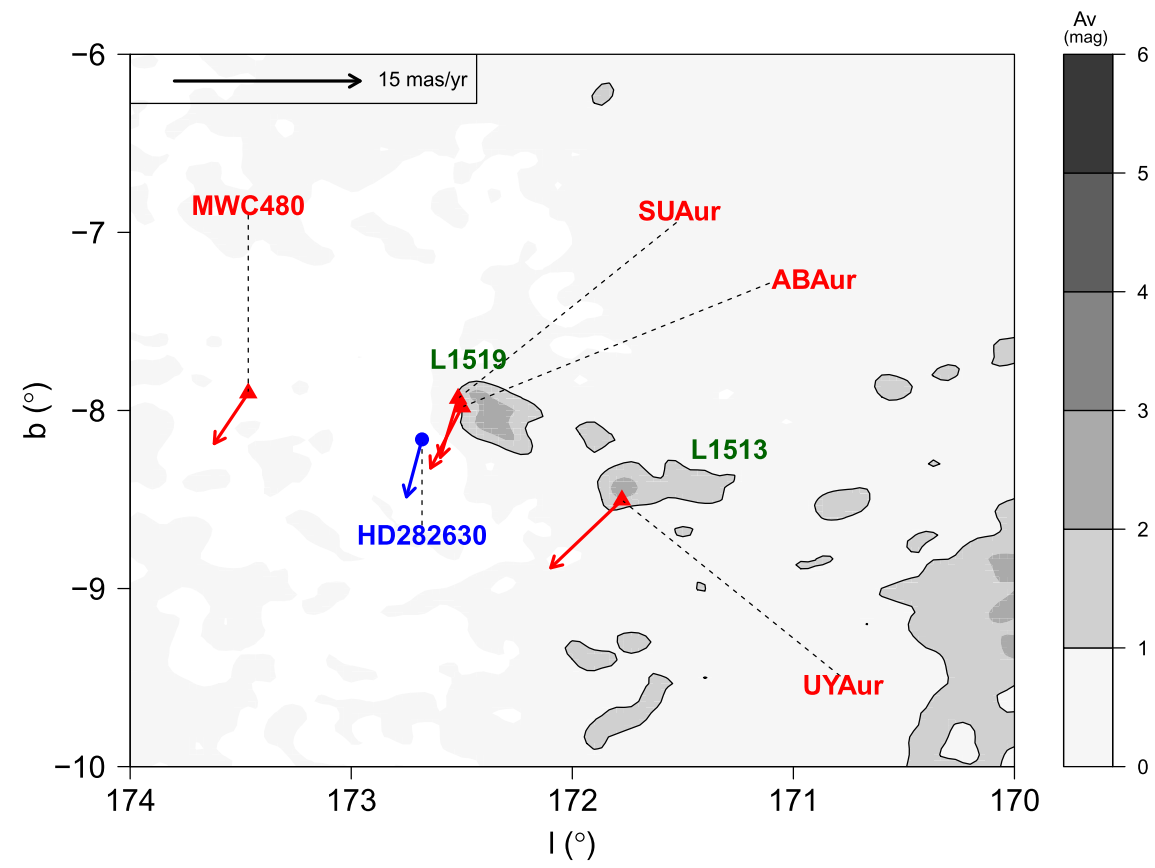

Figure 10. Structure of the $\mathrm{L} 1513\left(d=151_{-11}^{+13} \mathrm{pc}\right)$ and $\mathrm{L} 1519\left(d=142.1_{-2.3}^{+2.4} \mathrm{pc}\right)$ clouds overlaid on the extinction map from Dobashi et al. (2005). Blue circles and red triangles denote, respectively, the stars with VLBI and TGAS trigonometric parallax. The vectors indicate the stellar proper motions from Table 8 converted to the Galactic reference system and corrected for the solar motion (Schönrich et al. 2010) using the formalism described by Abad \& Vieira (2005).

located in Lynds 1519 (L1519; Lynds 1962). Another two stars (HD 282630 and MWC 480) are located in the surroundings of L1519 (see Figure 10), and their trigonometric parallaxes are also consistent with UY Aur, AB Aur, and SU Aur. The trigonometric parallaxes and spatial velocities of these sources are all consistent between themselves within the admittedly large errors. The weighted mean parallax is $\pi=7.014 \pm 0.113$ mas and yields a distance of $d=142.6_{-2.3}^{+2.3} \mathrm{pc}$, which we consider at this stage to be representative of both L1513 and L1519.

\subsubsection{Lynds 1531, 1534, and 1536}

V999 Tau and V1000 Tau are projected toward Lynds 1534 (L1534; Lynds 1962). The weighted mean parallax of these two sources is $\pi=7.215 \pm 0.110$ mas. This yields a distance estimate of $d=138.6_{-2.1}^{+2.1} \mathrm{pc}$. Our results also reveal that the nearby (in the plane of the sky) star-forming clumps Lynds 1531 (L 1531) and Lynds 1536 (L 1536) are located at different distances. The distance that we derive in this work for L1531 and L1536 is based solely on the trigonometric parallaxes of V807 Tau B and HP Tau G2 and represents a first distance determination to these clouds that will be refined when more data for the remaining cloud members becomes available. We find a distance of $d=126.6_{-1.7}^{+1.7} \mathrm{pc}$ and $d=162.7_{-0.8}^{+0.8} \mathrm{pc}$, respectively, for L1531 and L1536. This reveals a difference of about $36 \mathrm{pc}$ between these two clouds along the line of sight.

Based on the VLBI trigonometric parallaxes derived in this paper, we find that V807 Tau B is the closest star $(d=126.6 \pm 1.7 \mathrm{pc})$ in Taurus. The nominal distances obtained for V1096 Tau and V1098 Tau in L1495 indicate that they are somewhat closer than V807 Tau B, but the larger errors given in our solution due to the noncorrected binarity of V1096 Tau and the small number of detections for V1098 Tau make our results for these sources rather uncertain. On the other hand, we confirm HP Tau G2 as the remotest star $(d=162.7 \pm 0.8 \mathrm{pc})$ in the complex. Despite the different distances, we note that V807 Tau B and HP Tau G2 move with the same speed. However, their velocity vectors differ significantly, as the $w$-component of HP Tau G2 points to a different direction than most stars in our sample (see Table 9).

\subsubsection{Lynds 1551 and $L 1558$}

XZ Tau is projected toward Lynds 1551 (L1551; Lynds 1962) with a trigonometric parallax of $\pi=6.793 \pm 0.025$ mas, while UX Tau, located on the border of L1551 (see Figure 12), has a trigonometric parallax of $\pi=6.330 \pm 0.404$ mas. The weighted mean of these values yields $\pi=6.791 \pm 0.025$ mas and a distance estimate of $d=147.3_{-0.5}^{+0.5} \mathrm{pc}$. We note that this result is in good agreement with the distance of $d=148.7_{-0.9}^{+0.9} \mathrm{pc}$ obtained in this work for $\mathrm{T} \mathrm{Tau} \mathrm{Sb}$, which is projected toward the BDN 176.28-20.89 cloud (see Dobashi et al. 2005). We thus conclude that L1551 and BDN 176.28-20.89 are located at the same distance. Interestingly, this result also constrains the distance to the recently imaged HL Tau star (ALMA Partnership et al. 2015), which is also projected toward L1551 and located $<0.5^{\prime}$ from XZ Tau.

On the other hand, we note that DR Tau, which is projected toward Lynds 1558 (L 1558; Lynds 1962), has a trigonometric parallax of $\pi=4.820 \pm 0.424$ mas in the TGAS catalog. If we assume that the result delivered by Gaia-DR1 for this star is accurate enough, this will put DR Tau (and L 1558) in the background of the Taurus star-forming complex at a distance of $d=208_{-17}^{+20} \mathrm{pc}$. More study is clearly warranted in this regard, and the upcoming (and more precise) trigonometric parallaxes from Gaia-DR2 will allow us to confirm this scenario.

In addition to the stars mentioned in this section, we note that HD 28867 and HD 30171 are located in the vicinity 


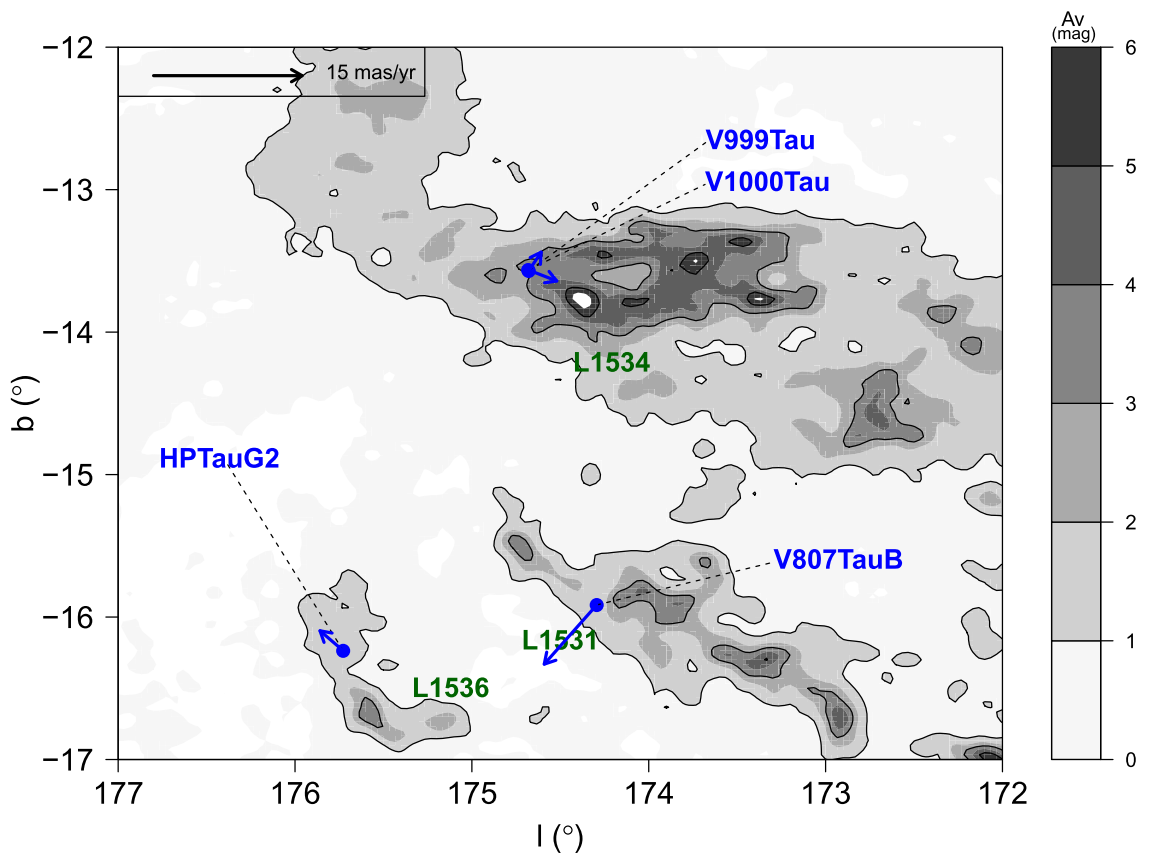

Figure 11. Structure of the L1531 $\left(d=126.6_{-1.7}^{+1.7} \mathrm{pc}\right)$, L1534 $\left(d=138.6_{-2.1}^{+2.1} \mathrm{pc}\right)$, and L1536 $\left(d=162.7_{-0.8}^{+0.8} \mathrm{pc}\right)$ clouds overlaid on the extinction map from Dobashi et al. (2005). Blue circles and red triangles denote, respectively, the stars with VLBI and TGAS trigonometric parallax. The vectors indicate the stellar proper motions from Table 8 converted to the Galactic reference system and corrected for the solar motion (Schönrich et al. 2010) using the formalism described by Abad \& Vieira (2005)

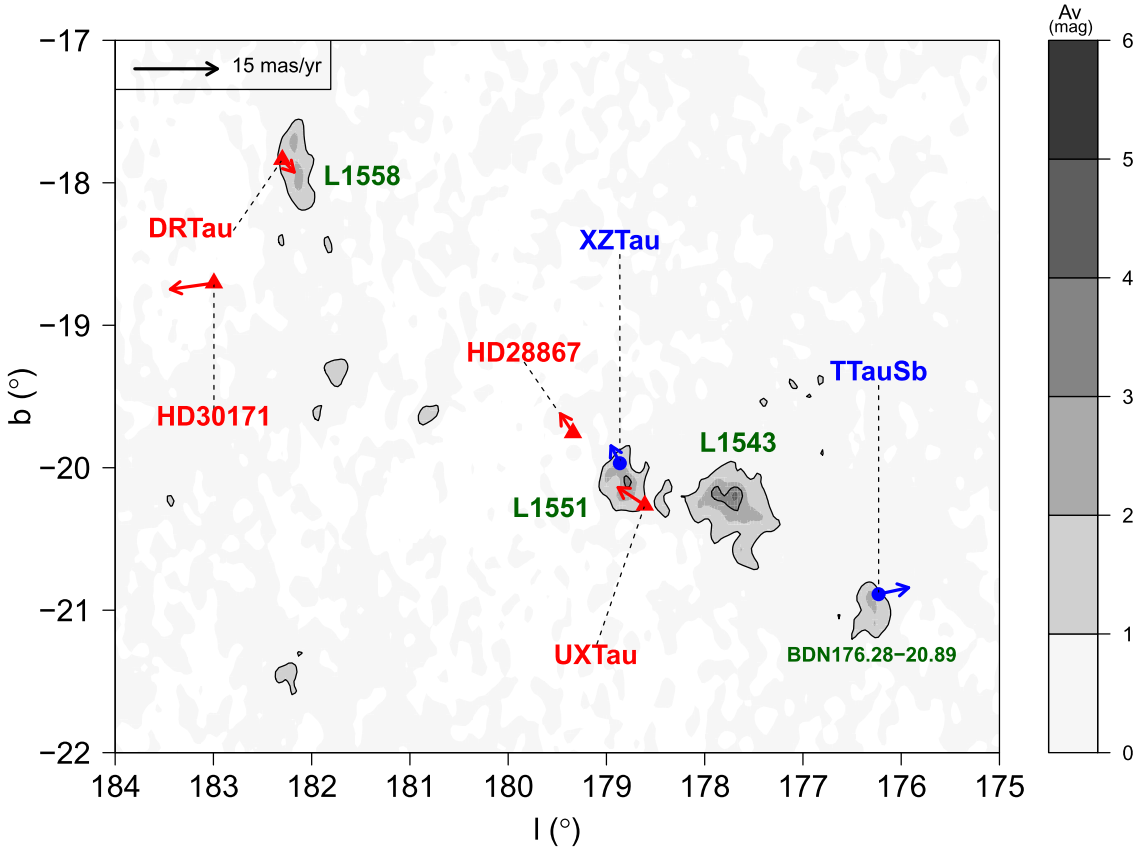

Figure 12. Structure of the L1551 $\left(d=147.3_{-0.5}^{+0.5} \mathrm{pc}\right)$ and L1558 $\left(d=208_{-17}^{+20} \mathrm{pc}\right)$ clouds overlaid on the extinction map from Dobashi et al. (2005). Blue circles and red triangles denote, respectively, the stars with VLBI and TGAS trigonometric parallax. The vectors indicate the stellar proper motions from Table 8 converted to the Galactic reference system and corrected for the solar motion (Schönrich et al. 2010) using the formalism described by Abad \& Vieira (2005).

of L1551 and L1558. HD 28867 has a radial velocity of $V_{r}=-14.70 \pm 7.40 \mathrm{~km} \mathrm{~s}^{-1}$, which is in obvious disagreement with the observed radial velocity of other group members (see Table 8). On the other hand, HD 30171 has a trigonometric parallax and spatial velocity that are more consistent with the properties of the L1551 cloud (see Tables 8 and 9), despite the closer proximity in the plane of the sky with L1558 (see also Figure 12). In a recent study, Kraus et al. (2017) performed a global reassessment of the membership status of known YSOs in Taurus and suggested the existence of a distributed older population of stars. HD 28867 was not included in their study, and HD 30171 was classified as a YSO candidate member, confirming that its membership status is rather uncertain. For these reasons, we have assigned HD 28867 and HD 30171 to an "off-cloud" population that will require further investigation with Gaia-DR2. 


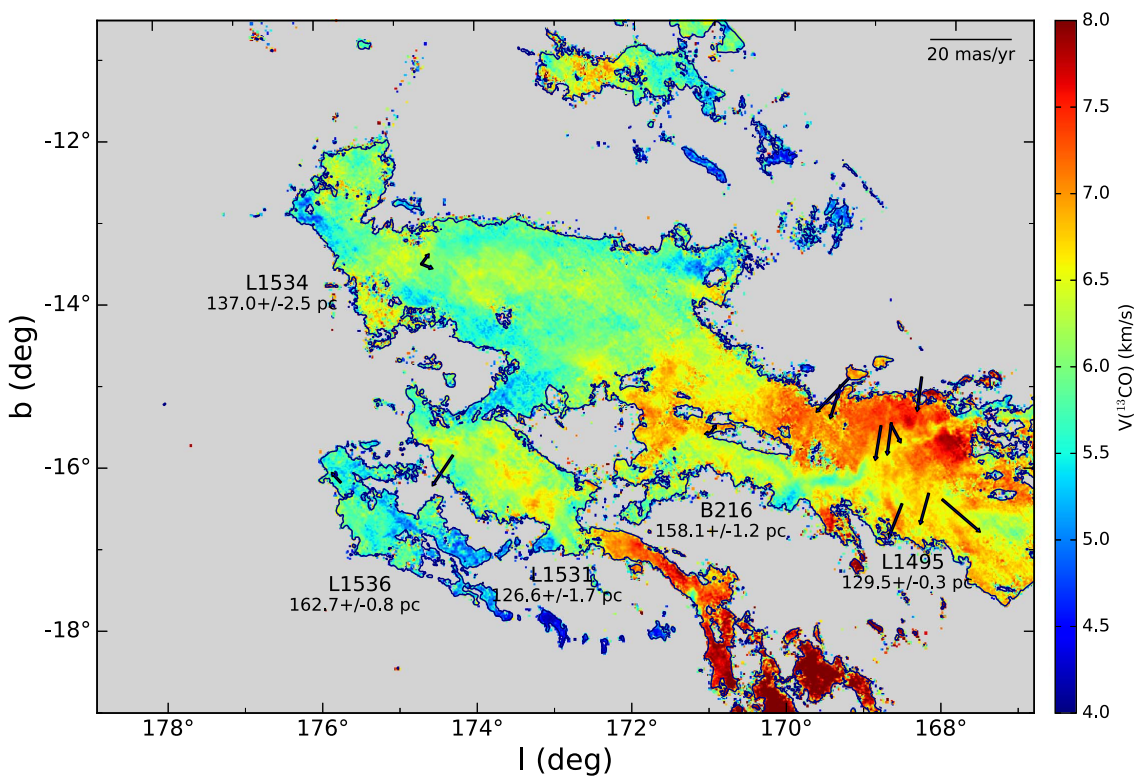

Figure 13. Location of the stars in our sample overlaid on the ${ }^{13} \mathrm{CO}$ map from Goldsmith et al. (2008). The vectors represent the stellar proper motions corrected for the solar motion (Schönrich et al. 2010) using the formalism described by Abad \& Vieira (2005). The most prominent star-forming clouds in the central portion of the complex and their distances are indicated in this diagram.

\subsubsection{Taurus (All Stars)}

In Table 10 we list the mean distance and spatial velocity derived for all stars in our sample (V1110 Tau, HD 28867 , HD 30171, and RY Tau are excluded from this analysis for the reasons discussed before). The mean distance of $d=141.8 \pm 0.2 \mathrm{pc}$ that we derive from our analysis is still consistent with the canonical distance estimate of $d=140 \pm 10 \mathrm{pc}$ (Kenyon et al. 1994), which is commonly used in the literature for the Taurus region. However, the resulting distance is only representative of a few clouds in the Taurus complex, and it does not reveal the important depth effects that exist in this region as demonstrated in our study. Interestingly, we conclude from Table 10 that the B216 clump in the filamentary structure of L1495 is moving at $(\Delta U, \Delta V$, $\Delta W)=(-1.7,-0.8,4.9) \pm(0.1,0.2,0.2) \mathrm{km} \mathrm{s}^{-1}$ with respect to the central part of the cloud, which implies a relative bulk motion of about $5.2 \pm 0.2 \mathrm{~km} \mathrm{~s}^{-1}$ between both structures. It is also interesting to note that L1551, which is the most southern cloud in our sample, is moving at $(\Delta U, \Delta V, \Delta W)=$ $(-1.6,-1.7,4.2) \pm(0.1,0.1,0.1) \mathrm{km} \mathrm{s}^{-1}$ with respect to L1495 and has a relative motion of $4.8 \pm 0.1 \mathrm{~km} \mathrm{~s}^{-1}$. On the other hand, we see from Table 10 that the spatial velocity for L1519 is fully consistent within $1 \mathrm{~km} \mathrm{~s}^{-1}$ of the mean spatial velocity computed for all stars in our sample.

We find that the dispersion of the spatial velocities among the various clouds is $\left(\sigma_{U}, \sigma_{V}, \sigma_{W}\right)=(2.4,2.5,2.1) \mathrm{km} \mathrm{s}^{-1}$. For comparison, the velocity dispersions derived from the proper motions converted to tangential velocities in right ascension and declination are 2.4 and $3.1 \mathrm{~km} \mathrm{~s}^{-1}$, respectively. This implies that the one-dimensional velocity dispersion in Taurus is somewhat higher than the value of $1 \mathrm{~km} \mathrm{~s}^{-1}$ adopted by Luhman et al. (2009) and smaller than the value of $6 \mathrm{~km} \mathrm{~s}^{-1}$ estimated by Bertout \& Genova (2006). This value is also similar to the one-dimensional velocity dispersion obtained by Dzib et al. (2017) and Kounkel et al. (2017) for YSOs in the Orion Nebula Cluster.

One interesting question that arises from our study is whether the stars and the molecular gas in this region exhibit the same kinematic properties. In this context, Goldsmith et al. (2008) performed a large-scale survey of the ${ }^{12} \mathrm{CO}$ and ${ }^{13} \mathrm{CO}$ molecular gas in Taurus, which we use here to further discuss our results. Figure 13 summarizes the distance of the various clouds in the central portion of the Taurus complex that we derive in this paper overlaid on the ${ }^{13} \mathrm{CO}$ velocity field produced in that survey. We extracted the ${ }^{12} \mathrm{CO}$ and ${ }^{13} \mathrm{CO}$ spectra at the position of the stars in our sample in a velocity interval from 3 to $13 \mathrm{~km} \mathrm{~s}^{-1}$, computed the centroid velocity of the molecular gas in each case, and estimated their errors from the rms of the individual spectra. We note that for some stars in our sample there was no apparent signal in one of the two spectra extracted from the ${ }^{12} \mathrm{CO}$ and ${ }^{13} \mathrm{CO}$ maps. So, we decided to restrict our analysis to the stars with measured centroid velocities from both spectra and took the weighted mean of the computed values as our final estimate for the velocity of the gas at the position of a given star. It is important to mention that this analysis is restricted to only nine stars in our sample with measured radial velocities in the literature (see Table 8) that are included in the region surveyed by Goldsmith et al. (2008) and fulfill this condition.

In Figure 14 we compare (i) the velocity of the molecular gas with the radial velocity of the stars (both are given with respect to the LSR) and (ii) the distance of the stars with the velocity of the associated gaseous clouds. First, we note that the velocity of the molecular gas (measured at the position of our sources) and the spectroscopic radial velocity of the stars are mostly consistent, confirming that the stars are indeed associated with the underlying gaseous clouds. It is important to mention that most stars used in this analysis are binaries, which explains both the existence of discrepant values (e.g., HD 283518) and the large errors on the radial velocities given in the literature (e.g., V1096 Tau). Second, we note that the velocity of the gas ranges from 6.5 to $7.5 \mathrm{~km} \mathrm{~s}^{-1}$ for most sources projected toward the central part of the L1495 cloud $\left(d=129.5_{-0.3}^{+0.3} \mathrm{pc}\right)$, and it varies from 6.0 to $6.5 \mathrm{~km} \mathrm{~s}^{-1}$ at the position of the remotest stars in this sample: V999 Tau $\left(d=143.4_{-3.9}^{+4.2} \mathrm{pc}\right)$, HD $283641\left(d=159.1_{-1.8}^{+1.8} \mathrm{pc}\right)$, and HP Tau $\mathrm{G} 2\left(d=162.7_{-0.8}^{+0.8} \mathrm{pc}\right)$. Although a perfect correlation between 


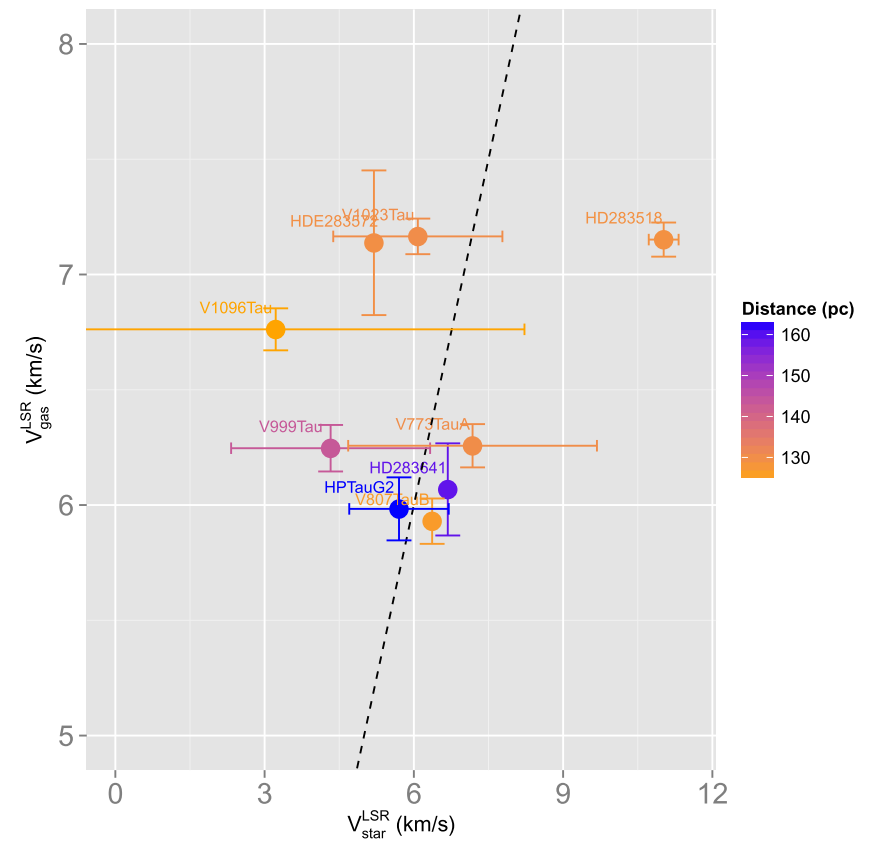

Figure 14. Comparison of the stellar radial velocity with the velocity of the ${ }^{12} \mathrm{CO}$ and ${ }^{13} \mathrm{CO}$ molecular gas measured at the position of each star. The colors indicate the distance of each star obtained in this study from VLBI astrometry. The dashed line indicates perfect correlation between the velocity of the gas and the radial velocity of the stars.

the distance of the stars and the velocity of the gas is not straightforward from Figure 14 (see, e.g., V773 Tau A and V807 Tau B), we found evidence that the observed velocity of the molecular gas at the position of our targets decreases with increasing distance of the star. This finding and the trigonometric parallaxes derived in this paper support our conclusion that the different structures of the Taurus complex are located at different distances. We will continue to investigate this issue using the upcoming parallaxes from Gaia-DR2 and a more significant number of stars to provide an accurate picture of the gas and stellar kinematics in this region.

Finally, we note that the angular size of the Taurus complex in the plane of sky is about $12^{\circ}$ in both Galactic longitude and Galactic latitude (see, e.g., Figure 1), which roughly corresponds to $30 \mathrm{pc}$ using the mean distance given in Table 10 below. From the closest (L1495) and remotest (L1536) molecular cloud with measured VLBI trigonometric parallaxes in this study (this excludes L1558), we estimate the depth of 33 pc. Thus, we conclude that the distance range in the plane of the sky and that in the line of sight are equivalent.

\section{Conclusions}

In this study we reported on multiepoch VLBI radio observations taken as part of the GOBELINS project in the Taurus star-forming region. We detected 26 YSOs with the VLBA and presented the astrometry of 18 stars (or stellar systems) in our sample. The absolute positions measured in this work were modeled to derive the trigonometric parallaxes and proper motions of both single stars and binaries to a few percent of accuracy. By combining our observations with data from previous studies in the literature, we were able to solve simultaneously for the astrometry and orbital motion of the sources in binary systems over an extended time base and provide a more accurate solution for the trigonometric parallax. Thus, our results are more accurate than the trigonometric parallaxes from Gaia-DR1 for both single stars and binaries, where the orbital motion of such systems was not taken into account. The VLBI trigonometric parallaxes presented in this paper are also more precise than the results from Gaia-DR1 by almost one order of magnitude. Our analysis also made it possible to determine the dynamical masses of the individual components in four systems (V1023 Tau, T Tau S, V807 Tau B, and V1000 Tau).

We converted the trigonometric parallaxes derived in this study into stellar distances and investigated the three-dimensional structure of the Taurus complex. We confirm the existence of significant depth effects and concluded that the various star-forming clouds of the complex are located at different distances. We found a mean distance of $129.5 \pm 0.3 \mathrm{pc}$ to the central part of the dark cloud L1495 and reported on the distance of $158.1 \pm 1.2 \mathrm{pc}$ toward the B216 clump in the filamentary structure of this cloud. Based on our VLBI observations, we conclude that V807 Tau B, which is projected toward L1531, is the closest star in our sample located at $126.6 \pm 1.7 \mathrm{pc}$. On the other hand, HP Tau G2 projected toward the nearby (in the plane of the sky) L1536 cloud is the farthest star in the complex located at $162.7 \pm 0.8 \mathrm{pc}$. Altogether, this implies a depth of about $36 \mathrm{pc}$ based solely on the distances derived from VLBI trigonometric parallaxes. In particular, we note that one of the clouds for which we derive a distance (Lynds 1551) contains the young star HL Tau, which has recently been the subject of many studies on the ALMA imaging of its protoplanetary disk. We argue that the distance derived here $(d=147.3 \pm 0.5 \mathrm{pc})$ should be used for any future study of that specific source.

Finally, we combined the stellar distances obtained in this paper with published radial velocities to compute the spatial velocities of Taurus stars. We verified that the one-dimensional velocity dispersion among the various clouds in the complex amounts to $2-3 \mathrm{~km} \mathrm{~s}^{-1}$. Moreover, we showed that the velocity of the molecular gas structures is somewhat smaller for the remotest stars in our sample $(d \simeq 160 \mathrm{pc})$ as compared to the closest stars projected toward L1495 ( $d \simeq 130 \mathrm{pc})$.

The distances produced by the GOBELINS project in Taurus represent one important step to map the three-dimensional structure of the complex with unprecedented accuracy and precision. In addition, they also provide us with an independent consistency check of the upcoming trigonometric parallaxes from Gaia-DR2 for the targets in common. We anticipate that we will soon be able to deliver more results for other targets in our sample (including binaries and multiple systems) that are currently being monitored by our team, and we will use GaiaDR2 trigonometric parallaxes to provide a more complete picture of the Taurus region.

P.A.B.G. acknowledges financial support from the São Paulo Research Foundation (FAPESP) through grants 2013/04934-8 and 2015/14696-2. L.L. acknowledges the financial support of DGAPA, UNAM (project IN112417), and CONACyT, Mexico. G.N.O.-L. acknowledges support from the Alexander von Humboldt Foundation in the form of a Humboldt Fellowship. M.K. acknowledges support provided by the NSF through grant AST-1449476 and from the Research Corporation via a Time Domain Astrophysics Scialog award (no. 24217).

Facilities: VLBA (NRAO)_-The National Radio Astronomy Observatory is operated by Associated Universities, Inc., under cooperative agreement with the National Science 
Foundation. The Long Baseline Observatory is a facility of the National Science Foundation operated under cooperative agreement by Associated Universities, Inc. DiFX correlator - This work made use of the Swinburne University of Technology software correlator, developed as part of the Australian Major National Research Facilities Programme and operated under licence. This work has made use of the computing facilities of the Laboratory of Astroinformatics (IAG/USP, NAT/Unicsul), whose purchase was made possible by the Brazilian agency FAPESP (grant 2009/540064) and the INCT-A.

Software: AIPS (Greisen 2003), emcee (Foreman-Mackey et al. 2013), astropy (Astropy Collaboration et al. 2013), novas (Barron et al. 2011).

\section{ORCID iDs}

Phillip A. B. Galli (1) https://orcid.org/0000-0003-2271-9297 Laurent Loinard (i) https://orcid.org/0000-0002-5635-3345 Gisela N. Ortiz-Léon (i) https://orcid.org/0000-00022863-676X

Marina Kounkel (i) https://orcid.org/0000-0002-5365-1267 Sergio A. Dzib (1) https://orcid.org/0000-0001-6010-6200 Lee Hartmann (1) https://orcid.org/0000-0003-1430-8519 Juana L. Rivera (i) https://orcid.org/0000-0002-7631-2620 John J. Tobin @i https://orcid.org/0000-0002-6195-0152

\section{References}

Abad, C., \& Vieira, K. 2005, A\&A, 442, 745

ALMA Partnership, Brogan, C. L., Pérez, L. M., et al. 2015, ApJL, 808, L3 Andrews, J. J., Chanamé, J., \& Agüeros, M. A. 2017, MNRAS, 472, 675 Astropy Collaboration, Robitaille, T. P., Tollerud, E. J., et al. 2013, A\&A, 558, A33

Barron, E. G., Kaplan, G. H., Bangert, J., et al. 2011, BAAS, 43, 344.14 Bertout, C., \& Genova, F. 2006, A\&A, 460, 499

Bertout, C., Robichon, N., \& Arenou, F. 1999, A\&A, 352, 574

Boden, A. F., Torres, G., Duchêne, G., et al. 2012, ApJ, 747, 17

Boden, A. F., Torres, G., Sargent, A. I., et al. 2007, ApJ, 670, 1214

Briceno, C., Hartmann, L. W., Stauffer, J. R., et al. 1997, AJ, 113, 740

Cambrésy, L. 1999, A\&A, 345, 965

Carrasco-González, C., Rodríguez, L. F., Anglada, G., \& Curiel, S. 2009, ApJL, 693, L86

Dame, T. M., Hartmann, D., \& Thaddeus, P. 2001, ApJ, 547, 792

Deller, A. T., Brisken, W. F., Phillips, C. J., et al. 2011, PASP, 123, 275

Dobashi, K., Uehara, H., Kandori, R., et al. 2005, PASJ, 57, S1

Duchêne, G. 1999, A\&A, 341, 547

Duchêne, G., Ghez, A. M., \& McCabe, C. 2002, ApJ, 568, 771

Duchêne, G., Ghez, A. M., McCabe, C., \& Weinberger, A. J. 2003, ApJ, 592, 288

Dzib, S. A., Loinard, L., Rodríguez, L. F., et al. 2015, ApJ, 801, 91

Dzib, S. A., Loinard, L., Rodríguez, L. F., et al. 2017, ApJ, 834, 139

Elias, J. H. 1978, ApJ, 224, 857

ESA 1997, The Hipparcos and Tycho Catalogues (ESA SP-1200; Noordwijk: ESA)

Forbrich, J., Dupuy, T. J., Reid, M. J., et al. 2016, ApJ, 827, 22

Foreman-Mackey, D., Hogg, D. W., Lang, D., \& Goodman, J. 2013, PASP, 125,306

Forgan, D., Ivison, R. J., Sibthorpe, B., Greaves, J. S., \& Ibar, E. 2014, MNRAS, 439, 4057

Gaia Collaboration, Brown, A. G. A., Vallenari, A., et al. 2016, A\&A, 595, A2 Goldsmith, P. F., Heyer, M., Narayanan, G., et al. 2008, ApJ, 680, 428

Gomez, M., Hartmann, L., Kenyon, S. J., \& Hewett, R. 1993, AJ, 105, 1927 Gontcharov, G. A. 2006, AstL, 32, 759

Goodman, J., \& Weare, J. 2010, Commun. Appl. Math. Comput. Sci., 5, 65

Gottlieb, D. M., \& Upson, W. L., II 1969, ApJ, 157, 611

Greenstein, J. L., \& Shapley, H. 1937, AnHar, 105, 359
Greisen, E. W. 2003, in Information Handling in Astronomy-Historical Vistas, Astrophysics and Space Science Library, Vol. 285, ed. A. Heck (New York: Springer), 109

Harris, R. J., Andrews, S. M., Wilner, D. J., \& Kraus, A. L. 2012, ApJ, 751,115

Hartmann, L. 2002, ApJ, 578, 914

Hartmann, L., Hewett, R., Stahler, S., \& Mathieu, R. D. 1986, ApJ, 309, 275

Herbig, G. H., \& Bell, K. R. 1988, Third Catalog of Emission-Line Stars of the Orion Population (Santa Cruz, CA: Lick Observatory)

Jackson, J. M., Rathborne, J. M., Shah, R. Y., et al. 2006, ApJS, 163, 145

Johnson, D. R. H., \& Soderblom, D. R. 1987, AJ, 93, 864

Joncour, I., Duchêne, G., \& Moraux, E. 2017, A\&A, 599, A14

Joy, A. H. 1945, ApJ, 102, 168

Kenyon, S. J., Dobrzycka, D., \& Hartmann, L. 1994, AJ, 108, 1872

Kenyon, S. J., Gómez, M., \& Whitney, B. A. 2008, in Handbook of Star Forming Regions, Volume I: The Northern Sky, ed. B. Reipurth (San Francisco, CA: ASP), 405

Kharchenko, N. V., Scholz, R.-D., Piskunov, A. E., Röser, S., \& Schilbach, E. 2007, AN, 328, 889

Köhler, R., Kasper, M., Herbst, T. M., Ratzka, T., \& Bertrang, G. H.-M. 2016, A\&A, 587, A35

Kohler, R., \& Leinert, C. 1998, A\&A, 331, 977

Köhler, R., Ratzka, T., Herbst, T. M., \& Kasper, M. 2008, A\&A, 482, 929

Kounkel, M., Hartmann, L., Loinard, L., et al. 2017, ApJ, 834, 142

Kraus, A. L., Herczeg, G. J., Rizzuto, A. C., et al. 2017, ApJ, 838, 150

Krist, J. E., Stapelfeldt, K. R., Hester, J. J., et al. 2008, AJ, 136, 1980

Leinert, C., Richichi, A., \& Haas, M. 1997, A\&A, 318, 472

Lestrade, J.-F., Preston, R. A., Jones, D. L., et al. 1999, A\&A, 344, 1014

Lindegren, L., Lammers, U., Bastian, U., et al. 2016, A\&A, 595, A4

Loinard, L., Mioduszewski, A. J., Torres, R. M., et al. 2011, RMxAC, 40, 205

Loinard, L., Torres, R. M., Mioduszewski, A. J., et al. 2007, ApJ, 671, 546

Luhman, K. L., Mamajek, E. E., Allen, P. R., \& Cruz, K. L. 2009, ApJ, 703, 399

Lynds, B. T. 1962, ApJS, 7, 1

Martin, E. L., Rebolo, R., Magazzu, A., \& Pavlenko, Y. V. 1994, A\&A, 282,503

Mason, B. D., Wycoff, G. L., Hartkopf, W. I., Douglass, G. G., \& Worley, C. E. 2001, AJ, 122, 3466

McCuskey, S. W. 1939, ApJ, 89, 568

Meistas, E., \& Straizys, V. 1981, AcA, 31, 85

Melis, C., Reid, M. J., Mioduszewski, A. J., Stauffer, J. R., \& Bower, G. C. 2014, Sci, 345, 1029

Menten, K. M., Reid, M. J., Forbrich, J., \& Brunthaler, A. 2007, A\&A, 474,515

Nguyen, D. C., Brandeker, A., van Kerkwijk, M. H., \& Jayawardhana, R. 2012, ApJ, 745, 119

Ortiz-León, G. N., Dzib, S. A., Kounkel, M. A., et al. 2017a ApJ, 834, 143

Ortiz-León, G. N., Loinard, L., Kounkel, M. A., et al. 2017b ApJ, 834, 141

Panopoulou, G. V., Tassis, K., Goldsmith, P. F., \& Heyer, M. H. 2014, MNRAS, 444, 2507

Pradel, N., Charlot, P., \& Lestrade, J.-F. 2006, A\&A, 452, 1099

Racine, R. 1968, AJ, 73, 233

Rebull, L. M., Padgett, D. L., McCabe, C.-E., et al. 2010, ApJS, 186, 259

Schaefer, G. H., Prato, L., Simon, M., \& Patience, J. 2014, AJ, 147, 157

Schaefer, G. H., Prato, L., Simon, M., \& Zavala, R. T. 2012, ApJ, 756, 120

Schmalzl, M., Kainulainen, J., Quanz, S. P., et al. 2010, ApJ, 725, 1327

Schneider, S., \& Elmegreen, B. G. 1979, ApJS, 41, 87

Schönrich, R., Binney, J., \& Dehnen, W. 2010, MNRAS, 403, 1829

Seidelmann, P. K. 1992, Explanatory Supplement to the Astronomical Almanac (Mill Valley, CA: Univ. Science Books)

Siess, L., Dufour, E., \& Forestini, M. 2000, A\&A, 358, 593

Simon, M., Ghez, A. M., Leinert, C., et al. 1995, ApJ, 443, 625

Straizys, V., \& Meistas, E. 1980, AcA, 30, 541

Torres, R. M., Loinard, L., Mioduszewski, A. J., et al. 2012, ApJ, 747, 18

Torres, R. M., Loinard, L., Mioduszewski, A. J., \& Rodríguez, L. F. 2007, ApJ, 671,1813

Torres, R. M., Loinard, L., Mioduszewski, A. J., \& Rodríguez, L. F. 2009, ApJ, 698,242

Ungerechts, H., \& Thaddeus, P. 1987, ApJS, 63, 645

van de Kamp, P. 1967, Principles of Astrometry (San Francisco: Freeman)

Wahhaj, Z., Cieza, L., Koerner, D. W., et al. 2010, ApJ, 724, 835

Wenger, M., Ochsenbein, F., Egret, D., et al. 2000, A\&AS, 143, 9

Woitas, J. 2003, A\&A, 406, 685

Xing, L. F. 2010, ApJ, 723, 1542

Xing, L.-F., \& Xing, Q.-F. 2012, A\&A, 537, A91 